This report was prepared as an acxount of work sponsored by an agency of the United States Government. Neither the United States Government nor any agency thereof, nor any of their employees, makes any warranty, express or implied, or assumes any legal liability or responsibility for the accuracy, completeness, or usefulness of any information, apparatus, product, or process disclosed, or represents that its use would not infringe privately owned rights. Reference herein to any specific commercial product, process, or service by trade namc, trademark, manufacturer, of otherwise does not necessarily constitute or imply its endorsement, recommendation, or favoring by the United States Government or any agency thereof. The views and opinions of authors expressed herein do not necessarily state or reflect those of the United States Government or any agency thereof.

\title{
A Fully Automated Radiochemical Preparation System for Gamma- Spectroscopy on Fission Products and the Study of the Intruder and Vibrational Levels in ${ }^{83} \mathrm{Se}$
}

Oliver Gordon Lien, III (Ph.D. Thesis)

Manuscript date: October 1983 
A Fully Automated Radiochemical Preparation System for Gamma-Spectroscopy on Fission Products and the Stu:y of the Intruder and Vibrational Levels in ${ }^{83} \mathrm{Se}$

By

Oliver Gordon Lien, III

BA (San Jose State University) 1970

BS (San Jose State University) 1976

MS (University of California, Davis) 1978

\section{DISSERT'ATION}

Submitted in partial satisfaction of the requirements for the degree of

DOCTOR OF PHILOSOPHY

in

Engineering-Applied Science

in the

GRADUATE DIVISION

of the

\section{UNIVERSITY OF CALIFORNIA}

DAVIS

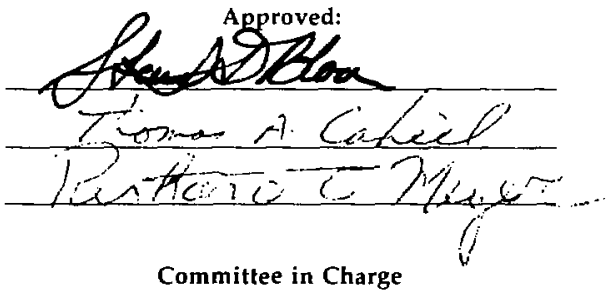

Deposited in the University Library 


\section{Table of Contents}

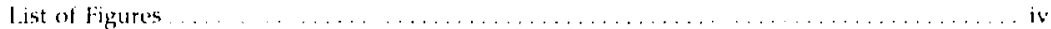

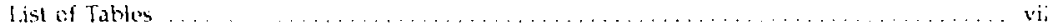

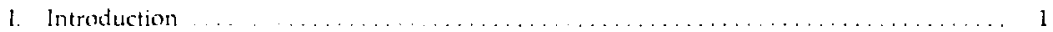

II. AUTOBATCH . ..................................... 2

Making the Batch Separation Process Competitive with
Continuous Separation Process $\ldots \ldots \ldots \ldots \ldots \ldots \ldots \ldots \ldots \ldots \ldots \ldots \ldots \ldots \ldots \ldots \ldots$

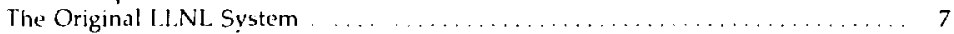

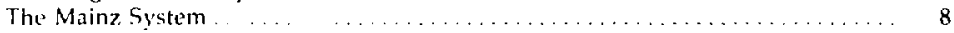

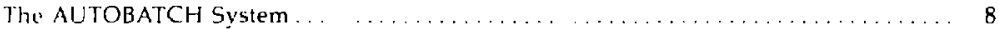

AUTOBATCH System Design ............................ 8

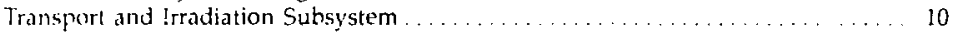

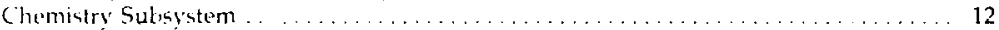

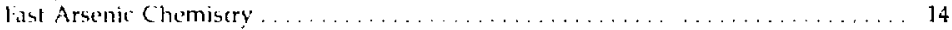

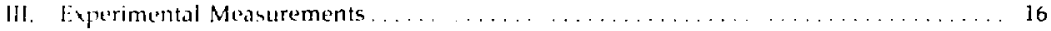

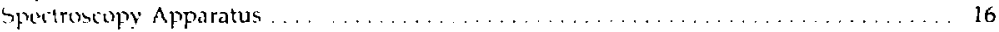

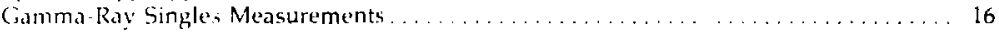

Gamma-Ray Coincidence Measurements ......................... 18

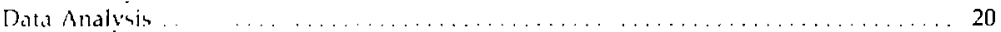

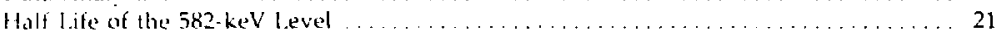

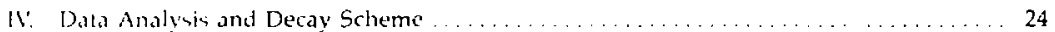

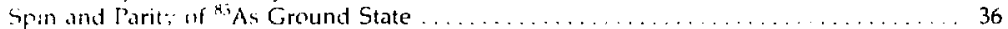

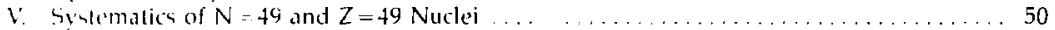

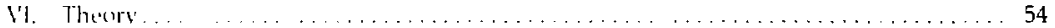

V'ibrational Model . . . . . . . . . . . . . . . . . . . . . . . . . . . . 54

Unified Model . . . . . . . . . . . . . . . . . . . . . . . . . . . . . . . . . 56

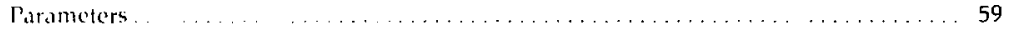

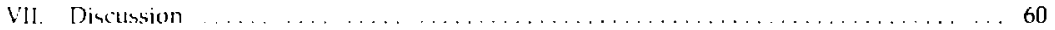

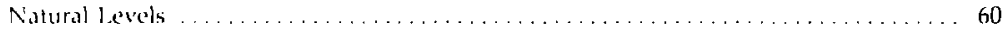

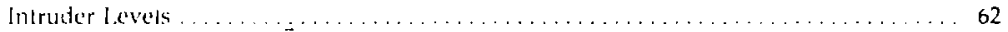

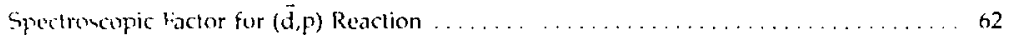

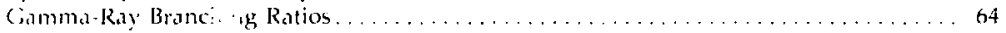

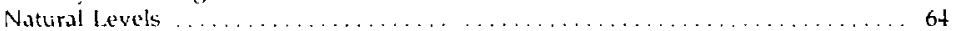

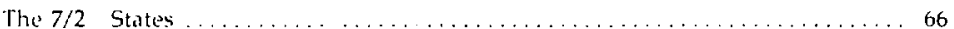

The Intruder levels . . . . . . . . . . . . . . . . . . . . . . . . 67

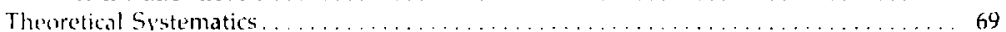

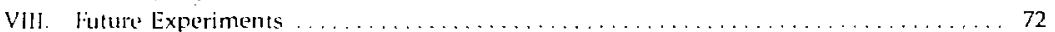

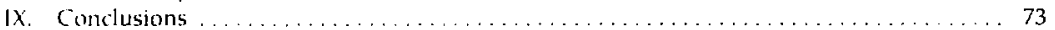

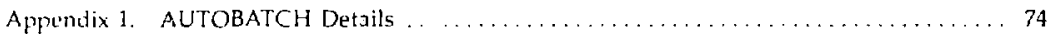

Transport Capsule . . . . . . . . 74

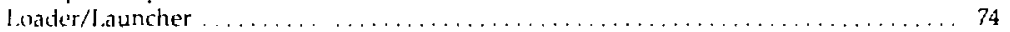

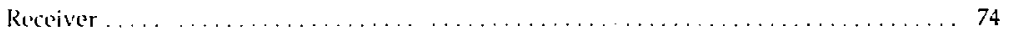

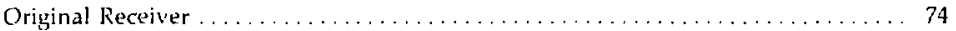

New receiver . . . . . . . . . . . . . . . . . . . . . . . . . . 75

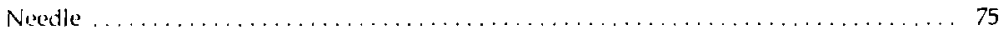

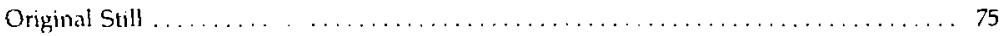

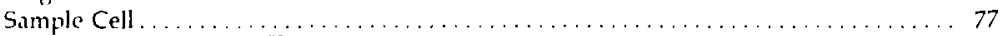

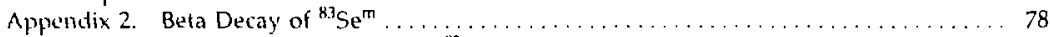

Appendix 3. Model Configurations for ${ }^{3} \mathrm{Se} \ldots \ldots \ldots \ldots \ldots \ldots \ldots \ldots \ldots \ldots \ldots$

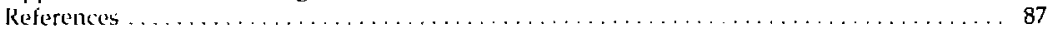

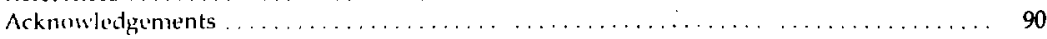




\section{List of Figures}

Fig. Il.1. Comparison of the fission vield curve for the thermal neutron fissioning of ${ }^{2} \mathrm{U}$ with the outpui of a typical on line isotope separator

Fig. II.2. IEfficiency of a batch processor as a function of $T_{n}$, the

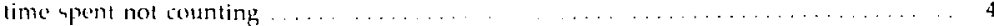

fig. 11.3. Plot of the ratio of the number of daughter counts to the number of

purcent counts, $N_{1} / V_{1}$, against the half-life ratio $\tau_{1} / \tau_{1} \ldots \ldots \ldots \ldots \ldots \ldots \ldots \ldots \ldots$

Fig. II.t. Schematir drawing of Mainz "building block" batch processor . . . . . . . . . . 9

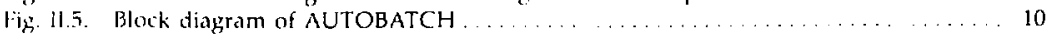

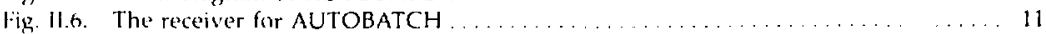

lig. II.7. The still and cyclone separator used in AUTOBATCH hydride chemistry ..... 13

lig. II.8. The AUTOBATCH chemistry system is shown schematically . . . . . . . . . 14

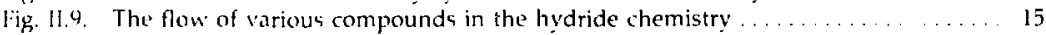

Fig. III.1. Top view of the chemistry/gamma spectroscopy interface $\ldots \ldots \ldots \ldots \ldots \ldots 18$

Hig. Ill.2. Plot of the $1455-\mathrm{keV}$ gate from the ${ }^{84} \mathrm{As}$ experiment . . . . . . . . . . . . . 19

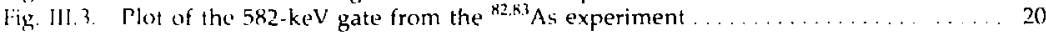

Fig. 111.4. Comparison of the $734-1113 \cdot \mathrm{keV}$ coincidence timing spectrum to a spectrum calculated with a 9.40 channel timing resolution .................. 23

Fig. III.j. Comparison of the 582-keV level TAC spectrum with spectra calculated with

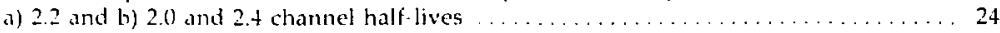

Fin. IV.1. Partial decay scheme showing gamma rays followed to determine

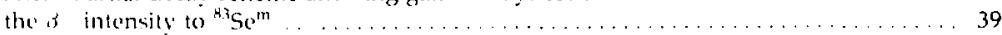

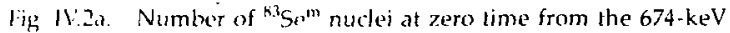
gamma Iransition

Figr. IV.2h. Number of ${ }^{\mathrm{H} .3 \mathrm{Sem}}$ nuclei at zero time from the $988-\mathrm{keV}$

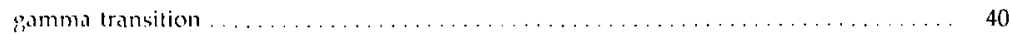

Fig. IV.3. Systematics of the nuclear levels of the odd-mass arsenics . . . . . . . . . . 43

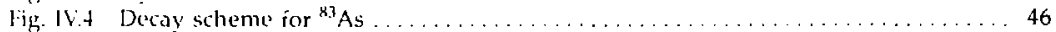

Fig. V.1. Systematics of the low-lying levels in ${ }^{11.3-119}$ In $\ldots \ldots \ldots \ldots \ldots \ldots \ldots \ldots$

Fig. V.2. Systematics of the energy of the first 2 level in the indium region $\ldots \ldots \ldots \ldots 2$

$\mathrm{Hi}_{\mathrm{f}}$. V.3. Systematics of the low-lying levels in the $\mathrm{N}=49$ isotones $\ldots \ldots \ldots \ldots \ldots \ldots . \ldots 3$

Fig. V.4. Systematics of the energy of the first $2^{+}$level in the $\mathrm{N}=50$ region ........ 54

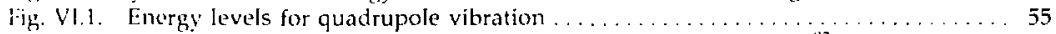

Hig. Vill.1. Comparison of experimental and theoretical level energies for ${ }^{83} \mathrm{Se} \ldots \ldots \ldots, 61$

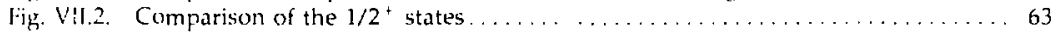

Fig. VIl.3. Comparison of spectroscopic factor for theory and experiment

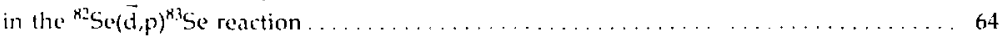

Fig. VII.4. Gamma transition branching ratios for theory and experiment

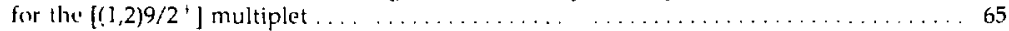

lig. VII.5. Gamma transition branchirg ratios for theory and experiment for the

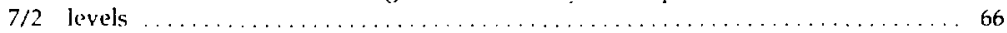

Fig. VIl.6. Ciamma transition branching ratios for theory and experiment for the

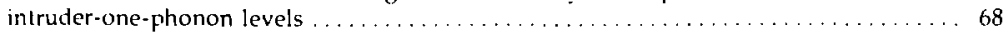

lig. VII.7. Gamma transition branching ratios for theory and experiment for the $3 / 2^{+}$

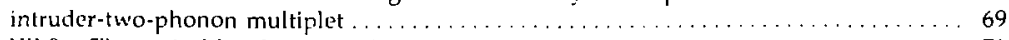

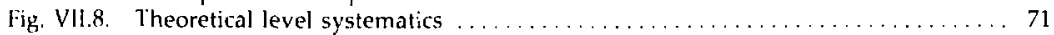

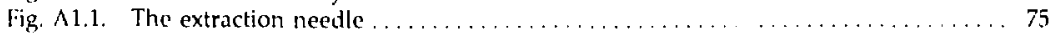

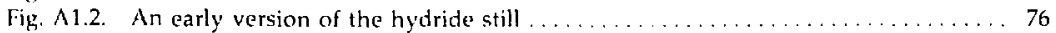




\section{List of Tables}

Table 1I.1. Computer controlled timing sequence for ${ }^{84}$ As $(5-s$ half-life $) \ldots \ldots \ldots \ldots \ldots$

Table III.la. Details of the gamma-ray spectroscopy experiments for

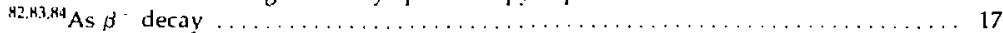

Tahl. 1Il.1b. Details of the coincidence gamma-ray spectroscopy experiments for

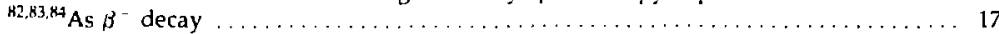

Table IV.1. Coincidences used in constructing the ${ }^{83}$ As decay scheme $\ldots \ldots \ldots \ldots \ldots .25$

Table IV.2. The basis for establishing each level in ${ }^{83} \mathrm{Se} \ldots \ldots \ldots \ldots \ldots \ldots \ldots \ldots \ldots 27$

Table IV.3. Placement of gamma transitions in the decay of ${ }^{83} \mathrm{As} \ldots \ldots \ldots \ldots \ldots \ldots \ldots 28$

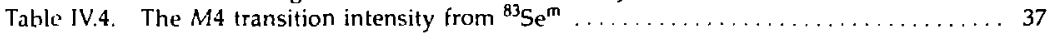

Table IV.5. Some values for selected beta branches from odd-mass arsenics. The beta transition of interest is from the arsenic ground state to the first

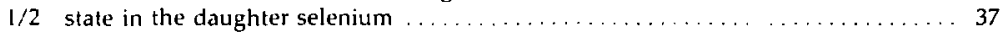

Table IV.6. Data used to determine the intensity of the $\beta^{-}$branch from ${ }^{\mathrm{H} 3}$ As to ${ }^{\mathrm{R3}} \mathrm{Se}^{\mathrm{m}}$

Table IV.7. Data development for evaluating the intensity of the $\beta^{-}$branch to the ${ }^{81} \mathrm{Se}^{\mathrm{m}}$ state

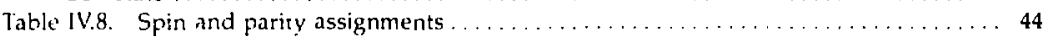

Table V.1. Systematics of the spectroscopic factor for the $(d, p)$ reaction to produce ${ }^{47} \mathrm{Se},{ }^{87} \mathrm{Kr}$ and ${ }^{87} \mathrm{Sr}$

Tabl. VII.1. Comparison of spectroscrupic factor distribution for theory and

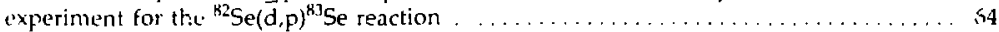

Tablt VII.2. Selected expansion coefficients for some odd parity states in ${ }^{83} \mathrm{Se} \ldots \ldots \ldots 6^{7}$

Table. VII.3. The parameters used for the $\mathrm{N}=49$ nuclei to reproduce the systematic behavior of the intruder and natural levels $\ldots \ldots \ldots \ldots \ldots \ldots \ldots \ldots$

Table VIl.4. The parumeters used for the $Z=49$ nuclei to reproduce the systematic behavior of the intruder and natural levels 


\title{
A Fully Automated \\ Radiochemical Preparation \\ System for Gamma- \\ Spectroscopy on Fission \\ Products and the Study of \\ the Intruder and Vibrational \\ Levels in ${ }^{83} \mathrm{Se}$
}

\begin{abstract}
AUTOBATCH was developed to provide a usable source of short-lived neutron-rich nuclides through chemical preparation of the sample from fission products for detailed gamma-ray spectroscopy, which would complement the output of on-line isotope separators. With AUTOBATCH the gamma rays following the $\beta^{-}$ decay of ${ }_{33}^{\mathrm{B} 3} \mathrm{As}_{5,1}$ were studied to determine:

- the ground state spin and parity of ${ }^{83}$ As to be $5 / 2^{-}$;

- the absolute intensity of the $\beta^{-}$branch from ${ }^{83} \mathrm{As}$ to ${ }^{83} \mathrm{Se}^{\mathrm{m}}$ to be $0.3 \%$;

- the absolute intensity of the ground state $\beta^{-}$branch from ${ }^{83} \mathrm{Se}^{\mathrm{m}}$ to ${ }^{83} \mathrm{Br}$ to be 39\%;

- the halflife of the $5 / 2_{1}^{+}$level to be $3.2 \mathrm{~ns}$;

- the structure of ${ }_{34}^{83} \mathrm{Se}_{49}$.
\end{abstract}

The results are used to show that the intruder structure which had been previously observed in the odd mass ${ }_{49}$ In isotopes could be observed in the $\mathrm{N}=49$ isotones. The observed structure is discussed in terms of the unified model calculations of Heyde which has been used to describe the intruder structure in the indium nuclei. The intruder structure is most strongly developed, not at core mid-shell, ${ }_{40}^{89} \mathrm{Zr}_{49}$, but rather at core mid-sub-shell ${ }^{83} \mathrm{Se}$. This difference is qualitatively understood to be due to the blocking of collectivity by the $Z=40$ subshell closure which prevents the intruder structure from occurring in ${ }^{87} \mathrm{Sr}_{49}$ and ${ }^{89} \mathrm{Zr}_{49}$.

\section{Introduction}

The only available source for neutron-rich nuclides from ${ }_{31} \mathrm{Ga}$ to ${ }_{71} \mathrm{Dy}$ is the fission pre cess. Lighter neutron-rich nuclides car be produced through spallation (GUS81) and fragmentation (SYM81). I designed and built AUTOBATCH to provide a usable source of the nuclides through chemical preparation of the sample from fission products for detailed gamma-ray spectroscopy.

$I$ also used AUTOBATCH to perform detailed studies of ${ }^{83} \mathrm{Se}_{49}$. The structure iit this nucleus was expected to be similar to the intruder structure seen in ${ }^{113-121}+49$. I used the unified model calculations of Heyde (HEY78, HEY80), which describe the indium nuclei, to describe the $N=49$ isotones with particular attention to ${ }^{\mathrm{B}} \mathrm{Se}$.

This thesis readily separates into three major sections. Chapter II, AUTOBATCH, describes the concept and design of the computer controlled nuclear-chemical sample preparation facility. In chapters III and IV, the experimental measurements and the development of the decay scheme 
for ${ }^{83} \mathrm{As}$ are described. In chapters V, VI, and Vi] I discuss the results obtained for ${ }^{\text {*3 }} \mathrm{Se}$ and the $\mathrm{N}=49$ isotones in light of the unified model developed by K. Heyde (HEY78, HEY80) and the results for the indium nuclei.

\section{AUTOBATCH: The LLNL Fast Chemistry System}

One of the few sources of neutron-rich, far-from-stability nuclei with atomic numbers ( $Z$ ) between 32 and 60 and with mass numbers $(A)$ between 80 and 160 is the fission process. To study any one of these nuclides, techniques must be devised that provide the best possible isolation of that nuclide from all other fission products. Radiochemical techniques offered the first widely used means of isolating nu-lides in this neutron-rich region and, as interest shifted to nuclei progressively farther from stability with progressively shorter half-lives, faster chemical techniques were developed. Isotope separation techniques also were developed to provide an even faster isolation of many fission products. How'ever, for a number of elements, the isotope separator give's an unusably low yield due to ion source problems (Fig. II.1) and rapid radiochemical separation is the only means of isolating these elements for study.

Basically there are two merhods that can be used to chemically isolate a radivactive sample, batch and continuous. Batch systems irradiate, purify and ther count a discrete sample, while continuous systems irradiate, purify and count incremental quantities of sample. This difference gives each system definite advantages and limitations. The advantages of a batch method are significant. Most important is the fact that data from a batch processor can be manipulated to retain half-life dependent information, which is more difficult with the equilibrium sample produced by a continuous separation process. The time dependence of the gamma activity due to the decay of nuclides $A$ and $B$ in the following sequence of decays

$\mathrm{A} \stackrel{\sigma}{\rightarrow} \mathrm{B} \stackrel{0}{\rightarrow} \mathrm{C}$

is significantly different in batch separation. Further, if there are several " $A$ " components, their signatures will be half-life dependent. By breaking the data acquisition period on a batch processor into discrete time periods and accumulating data in each time period (multichannelmultiscaling), it is easy to distinguish between the various components if the sample activity (short-lived, long-lived, daughter products). Acquiring $s$ fuential spectra further enhances the quality of the data for a short-lived is tope by placing most of these desired counts into the first time spectra and most of the counts from long-lived and daughter products into the later time spectra. Furthermore, the useful chemical separation reactions suitable for a batch processor, particularly those in aqueous solution, are much better known than those required for a continuous separation.

The major advantage of a continuous chemistry is that it offers shorter separation times through the use of instream chemistry. Continuous chemistry systems have been used for halflives down to $0.4 \mathrm{~s}$ (MAS80, ZEN78), while batch chemistries have been used for half-lives as short as $0.8 \mathrm{~s}$.

A secondary advantage of a continuous separation is that data can be acquired at a maximum rate at all times. Because a batch system processes discrete sample quantities, data cannot be acquired while the sample is being separated and the activity decreases as the sample decays while data is being accumulated; thus data can be acquired at the maximum rate only at the beginning of the acquisition period. I will compare the gross average count rates for these two systems. I will equate decay rate and count rate to make the analysis as general as possible.

To compare average count rate obtained from each system as a function of the half-life of the sample, J use a simple model based on the Bateman equations (FRI65) to determine the half-lives and recycle times for which a batch chemistry system would be competitive with a continuous chemistry system. Here, the pertinent factor is the overall average decay rate obtained with each 


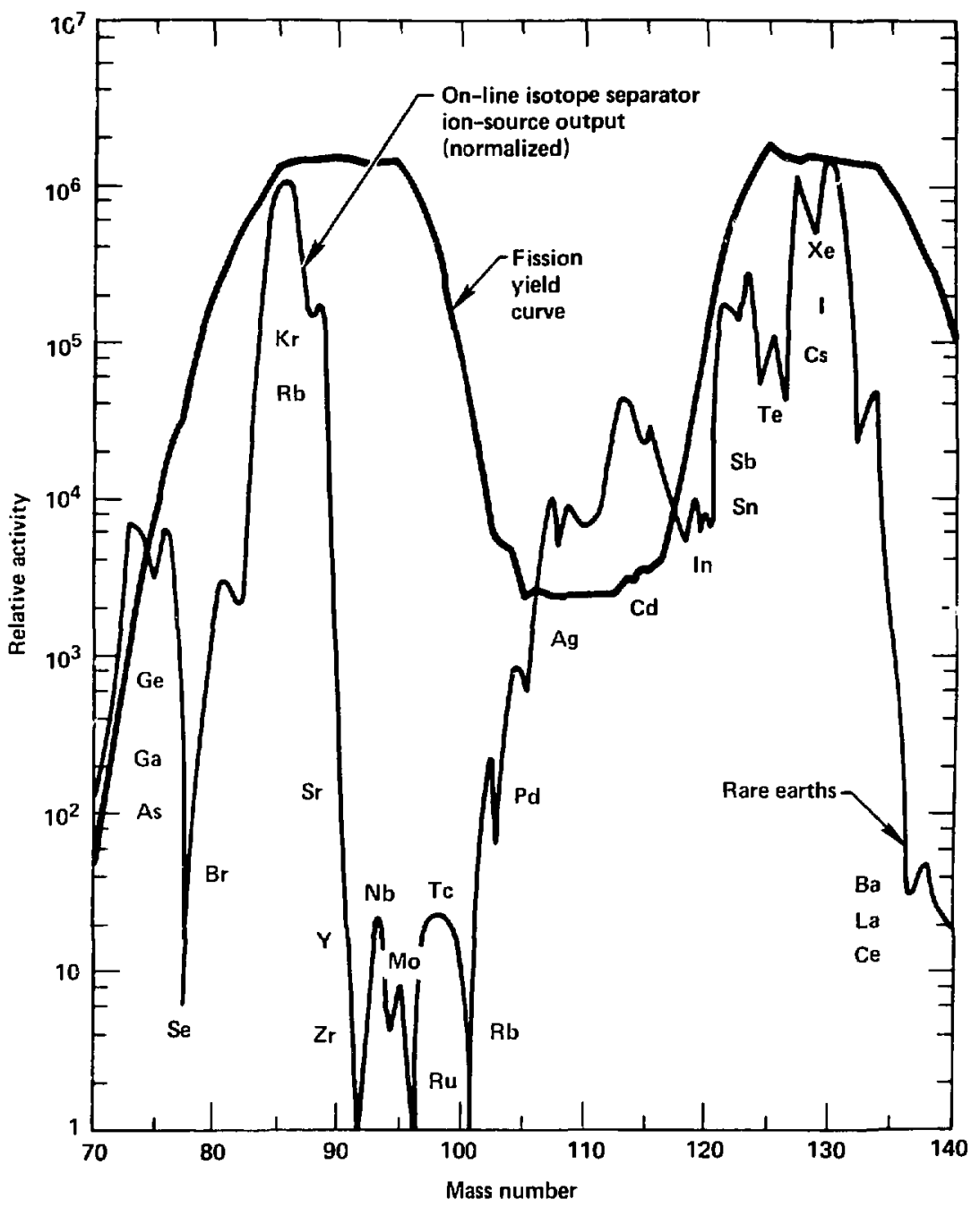

Fig. II.1. Comparison of the fission yield curve for the thermal neutron fissioning of ${ }^{235} U$ with the output of a typical on-line isotope separator. (LIE80) 
system. 1 consider a detector system which could be operated at a maximum count rate of $A_{m}$ the nuclide of interest has a half-life of $i_{\text {, }}$ and a decay constant of $\lambda_{1}\left(=\ln 2 / l_{i}\right)$. 1 assume that the continuous system operates at a constant count rate of $\Lambda_{m}$ (the maximum rate).

For the batch processor, two times are required to characterize the system, the time the detector in on, $T_{1}$, and the time the detector is off. $T_{n}$. Thus the average count rate of the batch system, $C_{b}$, is

$C_{1}=\lambda_{1 n} \frac{1-\exp \left(-\lambda_{t} T_{1}\right)}{\lambda_{1}\left(T_{1}+T_{n}\right)}$

For the batch process, if $T_{\mathrm{c}}$ and $T_{\mathrm{n}}$ are both twice $t_{\mathrm{r}}$, the efficiency $\left(C_{\mathrm{b}} / A_{\mathrm{m}}\right)$ is $27 \%$; if $T_{\mathrm{c}}$ is 2.5 halflives and $T_{n}$ is 4 half-lives, the relative efficiency drops to $18 \%$. In Eq. (II.2) there is a value of $T_{c}$ which maximizes $C_{h}$ for a given $T_{n}$. In Fig. Il.2 this maximum value of $C_{b}$ is plotted against $T_{n}$. This plot shows that if $T_{n}$ is held to two ur three half-lives, then an efficiency of $25 \%$ relative to the continuous method can be achieved.

This $25 \%$ efficiency is sufficient as a practical example illustrates. Antimony-134 has been studied with the LLNL AUTOBATCH system and with the Mainz batch and continuous chemistry systems. The experiments at Mainz took 4 weeks and 1 week respectively to obtain data of approximately equal quality. The experiment on the LLNL AUTOBATCH took 5 days to acquire significantly better data; indeed, only $2 \mathrm{~h}$ were required to identify the four known lines of ${ }^{134} \mathrm{Sb}$ and confirm the intensities observed at Mainz (LJE80).

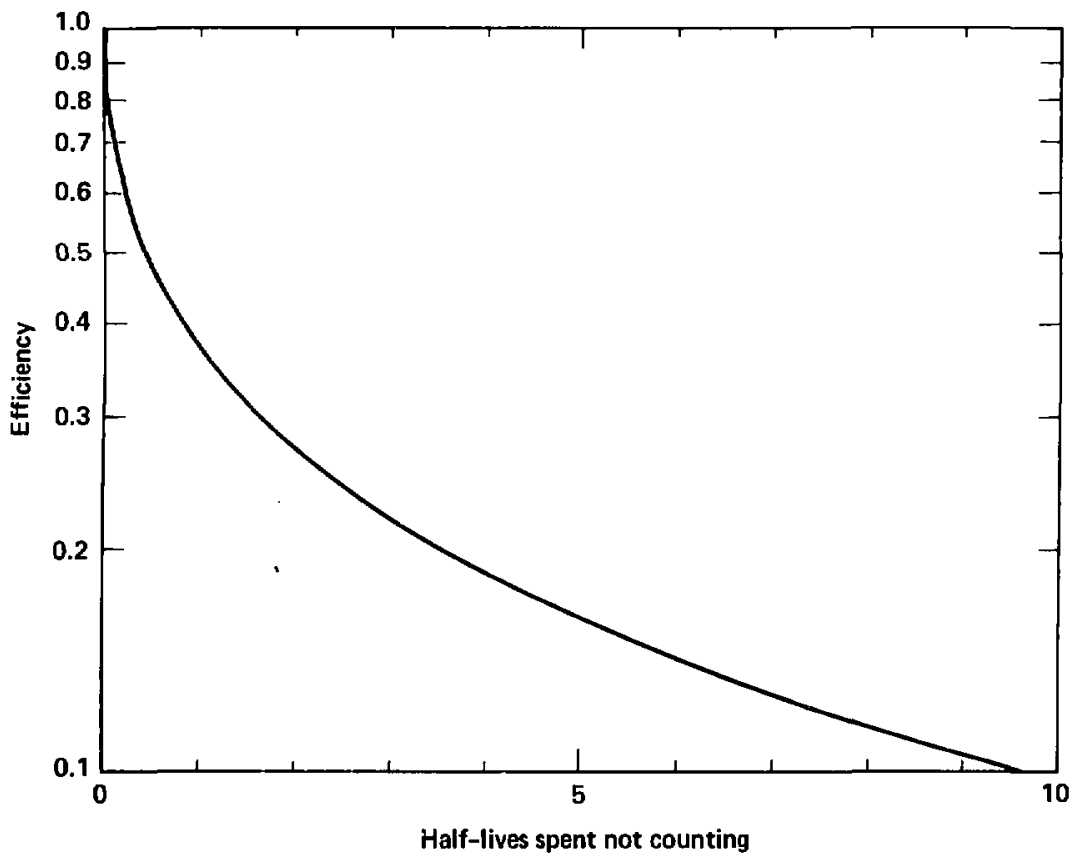

Fig. II.2. Efficiency of a batch processor as a function of $T_{n}$, the time spent not counting. For each value of $T_{n}$ the maximum value of eff( $\left.T_{c}\right)$ is plotted. 
However, samples chemically separated from fission products rarely have only a singlecomponent activity. Reasonable analysis of a multi ${ }_{\mathrm{i}}$ le-component sample requires consideration of both direct (parent) and daughter product activities of varying half-lives in addition to the desired activity. To understand the advantages and limitations of each type of system the production of various isotopes, desired and undesired, must be treated on an equal footing. For both types of systems I assumed that any isotope with a half-life shorter than that of the isotope of interest can be ignored. (In practice, sample counting begins only after most of the shorter-lived species have decayed away.) The activity from a nuclide $i$ at time $t_{1}$ during irradiation is

$A_{1}\left(t_{t}\right)=\lambda_{t} N_{1}\left(t_{1}\right)=Y_{1} R_{1} \exp \left(-\lambda_{1} t_{\mathrm{s}}\right)\left[1-\exp \left(-\lambda_{i} t_{t}\right)\right]$

where $r$, is the yield of the nuclide $i, R$, is the fission (or other production) rati, and $t_{\mathrm{s}}$ is the time between production of the activity and the beginning of counting. The yield can usually be taken as the fractional cumulative yield, however detailed consideration may require a more complex analysis.

In the batch system the value $\lambda_{1} T_{1}$ is small so that the initial source activity becomes

$A_{i}\left(T_{1}\right)=R_{i} \gamma_{1}, \lambda_{t} T_{1} \exp \left(-\lambda_{t} t_{\mathrm{s}}\right)$

where $A_{1}\left(T_{1}\right)$ indicates the activity after an irradiation of duration $T_{I}$ and separation time $t_{5}$. The decay rate of the directly produced activity is given by

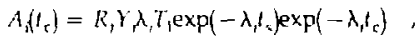

and the number of decays observed during the counting period of duration $T_{\mathrm{c}}$ is

$N_{1}\left(T_{1}\right)=R_{1} Y_{1} T_{1} \exp \left(-\lambda_{\mathrm{t}} t_{\mathrm{s}}\right)\left[1-\exp \left(-\lambda_{1} T_{\mathrm{c}}\right)\right]$

For the daughter products, $i$, of this activity, $i$, the decay rate is given by

$A_{\lambda}\left(t_{c}\right)=\frac{R_{1} Y_{1} \lambda_{t} \lambda_{1} T_{1} \exp \left(-\lambda_{1} t_{s}\right)}{\lambda_{1}-\lambda_{1}}\left(\exp \left(-\lambda_{1} t_{c}\right)-\exp \left(-\lambda_{1} t_{c}\right)\right)$

and the number of daughter decays observed is

$N,\left(T_{c}\right)=\frac{R_{1} i_{1} \lambda_{1} \lambda_{1} T_{1} \exp \left(-\lambda_{i} t_{s}\right)}{\lambda_{2}-\lambda_{1}} \times\left[\frac{1-\exp \left(-\lambda_{1} T_{c}\right)}{\lambda_{1}}-\frac{1-\exp \left(-\lambda_{1} T_{c}\right)}{\lambda_{1}}\right]$.

For the continuous system only the activity equations are necessary. The intent of the continuous system is that it be operated for $t_{1}$ much longer tianii any of the half-lives involved, thus the activity equation (Eq. (II.3)) becomes for the direct activities

$A_{1}\left(t_{1}\right)=R_{1} Y_{1} \exp \left(-\lambda_{i} t_{s}\right)$

For the daughter products of the continuous processor the activity is given by

$A_{f}\left(t_{i}\right)=\frac{R_{f} Y_{1} \exp \left(-\lambda_{i} t_{s}\right)}{\lambda_{1}-\lambda_{l}}\left[\lambda_{i}-\lambda_{j}-\lambda_{1} \exp \left(-\lambda_{i} t_{c}\right)+\lambda_{i} \exp \left(-\lambda_{i} t_{c}\right)\right]=R_{f} Y_{i} \exp \left(-\lambda_{l} t_{s}\right)$.

In Fig. II.3, I present the ratio $N_{j} / N_{i}$ as a function of the half-life ratio $\tau_{j} / \tau_{i}$ for a batch processor. The sample is to be counted for a period $2 \tau_{i}$. For a continuous processor the ratio $N_{i} / N_{i}$ equals oric. 
With these equations I can consider a three component system: the desired isotope, $t$, its daughter, $j$, and a longer lived isotope, $k$. The most important information is the relative rate at which decays of the desired isotope are observed. For the batch processor the limiting activity is the activity at zero time from these icotopies.

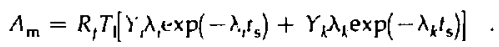

This relationship determines $R_{l} T_{\mathrm{I}}$ in Eqs. (II.6) and (II.8) giving the average usable decay rate for the desired nuclide

$C_{h}=A_{m} \frac{\left(1-\exp \left(-\lambda_{t} T_{s}\right)\right)}{\lambda_{1}\left(T_{c}+T_{n}\right)}\left[1+\frac{\lambda_{k} Y_{k}}{\lambda_{i} Y_{s}} \exp \left(\left(\lambda_{1}-\lambda_{k}\right) t_{s}\right)\right]^{-1}$

For the cor ....... . processor the limiting activity is

$$
A_{a t}=R_{l}\left[2 Y_{1} \exp \left(-\lambda_{l} t_{s}\right)-Y_{l} \exp \left(-\lambda_{\lambda} t_{s}\right)\right]
$$

which determines the value $R_{1}$ in Eys. (II.8) and (11.9), yielding an average observed decay rate for the desired nuclide, $i$,

$C_{c}=A_{\mathrm{m}}\left[2+\frac{Y_{h}}{Y_{1}} \exp \left(\left(\lambda_{\mathrm{t}}-\lambda_{\mathrm{k}}\right) t_{\mathrm{s}}\right)\right]^{\mathrm{l}}$.

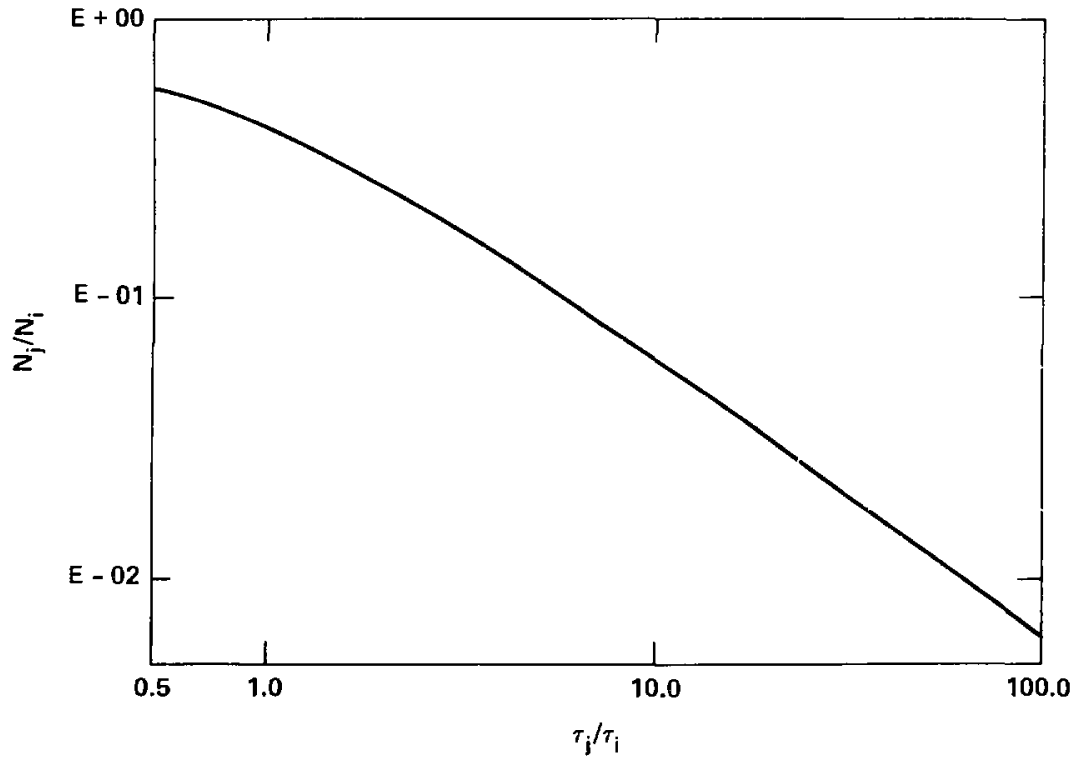

Fig. II.3. Plot of the atio of the number of daughter counts to the number of parent counts, $N_{j} / N_{i}$ (from Eqs. 11.6 and 11.8), against the half-life ratio $\tau_{j} / \tau_{i}$. This plot shows that the batch processor has considerably less daughter contamination than the continuous processor. For the continuous system (Eqs. 11.9 and 11.10 ) $N_{j} / N_{i}$ equals one. 
As an example, assume that $\lambda_{1}=\lambda_{k}=0.2 \lambda_{l}, t_{s}=0.2 T_{c}, \lambda_{t} T_{n}=\lambda_{1} T_{c}=2 \ln 2$, and $Y_{i}=Y_{k}$. With these conditions the average decay rate for the desired nuclide for the batch process is $70 \%$ of that average for the continuous process. Further the half-life of the daughter, $j$, has no effect on this ratio and the fraction of desired decays are substantially higher for the batch process than for the continuous process, $64 \%$ to $30 \%$. respectively.

Breaking the total acciuisition period into shorter periods with the data recorded into different spectra in each period further improved the data acquired on the batch system. Equations (II.6) and (1I.8) show that this results in the desired data being cc acentrated in the early spectra and the undesired data being placed into later spectra. This procedure has no effect on the continuous process as the activities are time independent. For the continuous system the value of $t_{\mathrm{s}}$ can be varied to differertiate between direct activities of differing half-lives, but will not differentiate between daughter and parmt as the daughter decay constan: toes not enter into the activity in Eq. (II.10)

The sample produced by the continuous separation is effectively an equilibrium sample. The equations show that the costribution to the continuous proress from daughter products is nearly equivalent to the contribution from the desired isotope and the contribution of longer lived species differs only by the ratio $\gamma_{1} / r_{\text {. }}$.

From the above analysis. I conclude that the batch process is competitive with the continuous method for isotopes with half-lives down to apf roximately $5 \mathrm{~s}$. For isotopes with ?.? lives shorter than thi, it is unlikely that a batch separation system could recycle within two to three half-lives of the desired nuclide which is required to keep the average count rate competitive with a contirulous syatem.

\section{Making the Batc' Separation Process Competitive with Continuous Separation Process}

There are many batch process chemistries a ailable for isolating a radioactive sample from rissio: puducts. (See HER69. MEY79a, and TRA78 for reviews of fast chemistry.) Here I discuss two irath . hemistry systems that use these frocesses for studying short-lived fission products. $P_{i, n}$ if these systems had their good points, but neither could meet the criteria established in the previous section for an efficient system for nuclides : vith half-lives less than $1 \mathrm{~min}$. Trautmann and Hermann (see [RA78) at the Institüt für Kernchemie der Universitat Mainz pioneered fast batch chemistries with separations as fast as $1 \mathrm{~s}$. However, their recycle time was $20 \mathrm{~min}$. Another batch system was developed at LLNL by Meyer and Landrurn. Thi- system made use of slower chemistries with separations of $45 \mathrm{~s}$, but had a recycle time $3 \mathrm{~min}$. One of the aims of my thesis project was 10 ar struct a batch-process chemistry system suit, ble for studying short-lived species which combined the fast separations simila: :o tnusi used in Mainz with the rapid recycling developed at LI.NL.

\section{The Original LLNL System}

The batch processor at LI.N! was used for detailed nuclear spectroscopic measurements. The source of fission products was uranum samples irradiated in a 3-MW pool-type reactor as fast as the separation system could process thern. Although this system was largely automated, the individual components vere slow and not integrated. Each uranium sample had to be manually loaded into the irradiation and transport system. When the irradiated sample arrived at the chemistry station, the prograni controlling the automatic separation frocess had to be manually started via a command entered at a teletypewriter. After the signal was given, it took more than a second to extract the sample from the transport capsule. Valves that controlled the reagent flow took tenths of seconds to respond. Reaction vessels were very large, up to $200 \mathrm{ml}$, requiring excessive amounts of time to transfer solutions. The sample separation was automated, but once the sample was separated, it had to be transferred manually to the detector location. Afrer the sample was taken to the detectors, the chemistry system was automatically cleaned, reconditioned, and refilled; only a new container for the final samplo had be to inserted manually before the next cycle. 
Thus, although the LI.NL system was slow, the components were basically amenable to automation,

One other drawhack to the original LLNL batch processor was that the failure rate was high. Parts failed frequently: valves stuck, the irradiation and transport subsystem jammed, etc., resulting in considerable $k$ st time. Thus, the reliability and durability had to be improved for the system to run efficiently for short-lived samples. With a goal of 20 chemically separated samples per hour, a $10 \%$ failure rate at $5 \mathrm{~min}$ down time per failure, the actual number of samples processed is $86 \%$ of the goal. As the number of samples processed per hour increases, the penalty for failure becon:es increasingly severe. When the target rate is 60 samples per hour, the $10 \%$ failure rate drops production to $67 \%$; at 120 samples per hour, production drops further to $50 \%$. Thus, reliability and durability become increasingly cruciai features as the sample half-life decreases.

\section{The Mainz System}

The Mainz batch system was used to measure the half-ljyes and major transitions for ${ }^{84} \mathrm{As}$ $(5 \mathrm{~s}),{ }^{185} \mathrm{As}(2 \mathrm{~s})$, and ${ }^{104} \mathrm{Nb}(0.8 \mathrm{~s})$. (See TRA78 for a complete list of their chemistries.) The source of fission products was uranium sample irradiated in a TRIGA reactor which could cnly be pulsed every $20 \mathrm{~min}$. At this pace, there was sufficient time to clean, recondition, refill. and reassemble the chemistry apparatus by hand. Thus the Mainz chemistry sysiem shown in Fig. II.4 incorporatrd many strictly wanual features. Stopcocks were used for valves, syringes injected measured quantities of chemicals, and zinc metal powder was used for a reagerit, none of which were suitable for automation. The earlier efficiency analysis shows that for ${ }^{84} \mathrm{As}$ this system was $0.6 \%$ efficient . erative to a coninuous system.

\section{'The AUTOBATCH System}

A new automated batch chemistry system AUTOBATCH was built taking advantage oi scme of the existing components of the LLNL batch processor, as well as the fast separation chemistries develops.' at Mainz. The goals of this system were: fast sample preparation of approximately $1 \mathrm{~s}$, rapid recycle time of about $15 \mathrm{~s}$, and high reliability and durability with about 1000 cycles in $8 \mathrm{~h}$.

To achic re these goals, I elected to use standard commercially available components wherever possible. For example, many solenoid valves were used in the system and the commercially available ones were found to be faster, more reliable, and easier to replace than custom-designed valves. It was also obvious that moving parts had to be minimized. Where possible, the moving part was eliminated. If this was not possible, the extent of the required motion was minimized and the component redesigned in such a way that the action was not time-critical.

Most importantly, the system had to be fully automated and integrated. All steps, from irradiation, through separation, to data acquisition and recycling, were computer-controlled. Th. 15 I eliminated all manually controlled components. Hands-on steps (e.g., changing drying tubes and r filling reagent reservoirs) could be required no more frequently than every $15 \mathrm{~min}$, not for every sample. Such complete automation required that all components be completely integrated. The sample transport, chemistry, and data acquisiton subsystems had to communicate in such a way that changes in one subsystem did not require changes in all the others.

\section{AUTOBATCH System Design}

The AUTOBATCH system (Fig. U.5) was designed with total automation as a major design criterion. The system was conceived as a central controller with three major subsystems: sample transport and irradiation, chemistry, and data acquisition. These three subsystems would be linked through the controller but otherwise independent.

The sample cycle was designed to proceed as follows. The controller would initiate the sampie cycle by starting the irradiation and transport cycle and simultaneously signalling the chemistry system that sample irradiation had begun. When the irradiated sample arrived at the 


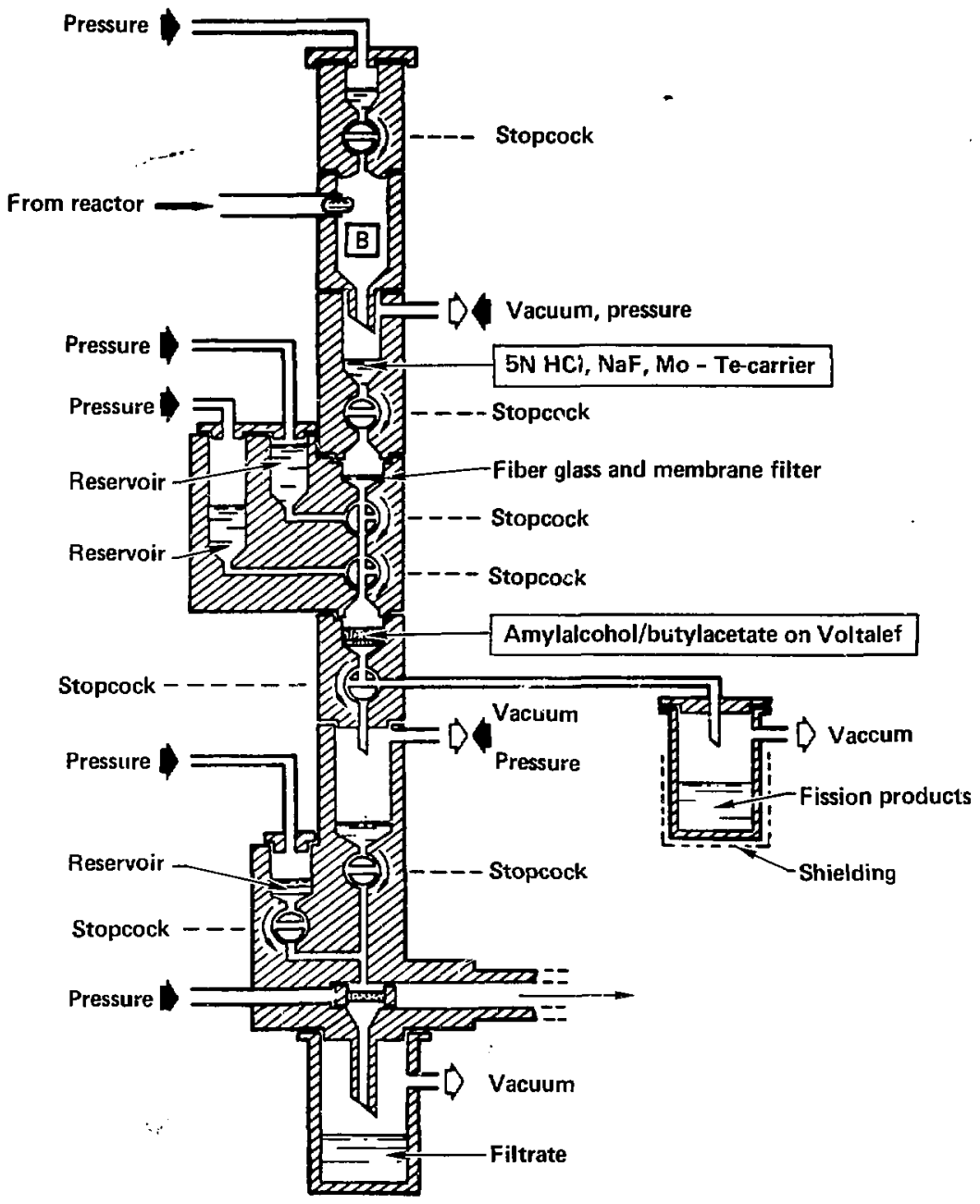

Fig. II.4. Schematic drawing of Mainz "building block" batch processor. The pieces require considerable machining. The stopcocks must be manually reset and the reservoirs manually refilled after prucessing each sample. (Adapted from TIT77.) 


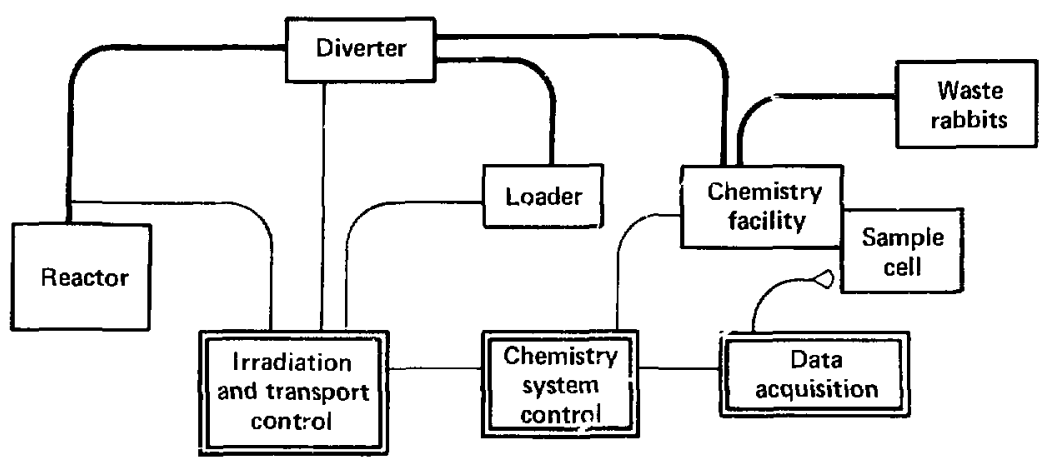

Fig. II.5. Block diagram of AUTOBATCH. Sample transport lines are shown in heavy lines, computer control and data lines are shown in light lines.

receiver, the raw fission product sample was extrarted from the transport capsule and the chemistry sequence bigan. Sample separation and processing was completed when the finished sample arrived at the detector location. The chemistry subsystem then signalled the data acquisition system via the controller to start counting the sample. While the sample was being counted, the chemistry apparatus was automatically cleaned and reconditioned, the used sample transport capsule was ejected, and the next capsule was loadecl. The chemist:y subsystem was - inalied by the controller when the data acquisition sequence was completed and the sample ct. was then cleaned and reconditioned. At this point, AUTOBATCI I was ready to process the nex: sample.

The entire irradiation, separation, and data acquisition sequence would take place without interruption and without intervention. The orily required nanual steps were to refill the sample hopper at the loader-launcher every 35 samples, which did not interrupt the sample processing, and to refill the reagent reservoirs (every 30 to $60 \mathrm{~min}$ ), which required less than $5 \mathrm{~min}$ of down time.

\section{The Transport and Irradiation Subsystem}

The transport and irradiation subsystem consisted of four major components all under computer control: the loader-iauncher, the diverter, the irradiation station, and the receiver (see Fig. 1I.6). The ioader-launcher hopper held 38 sample capsules; capsules were loaded via a pneumatically activated breech mechanism. Mirroswitches detected the breech position (open, closed, or in between) and a photosensor confirmed the loaded/not loaded status. The actual loading sequence required $5 \mathrm{~s}$. A sccond photosensor monitored the number of capsules in the hopper and triggered an audible alarm when there were only three capsules left. To save time the load sequence was executed during the cleanup sequence of the chemistry subsystem.

The diverter directed the sample capsule to its proper destination. It was moved by pneumatic cylinders and its proper positioning was confirmed by microswitches. Positioning required $0.3 \mathrm{~s}$.

The irradiation station was located adjacent to the reactor core. The sample capsule was sent pneumatically 10 a stop at the bottom of the transport tube and remained there during the preprogrammed irradiation period. The irradiated capsule was then ineumatically ejected from core and sent to the chemistry facility.

The receiver was located at the chemistry facility. The irradiated sample capsule was sent to the receiver where it was impaled by its own momentum on the extraction needle. The gas pressure in the pneumatic tube behind the capsule prevented it from rebounding off the needle. 


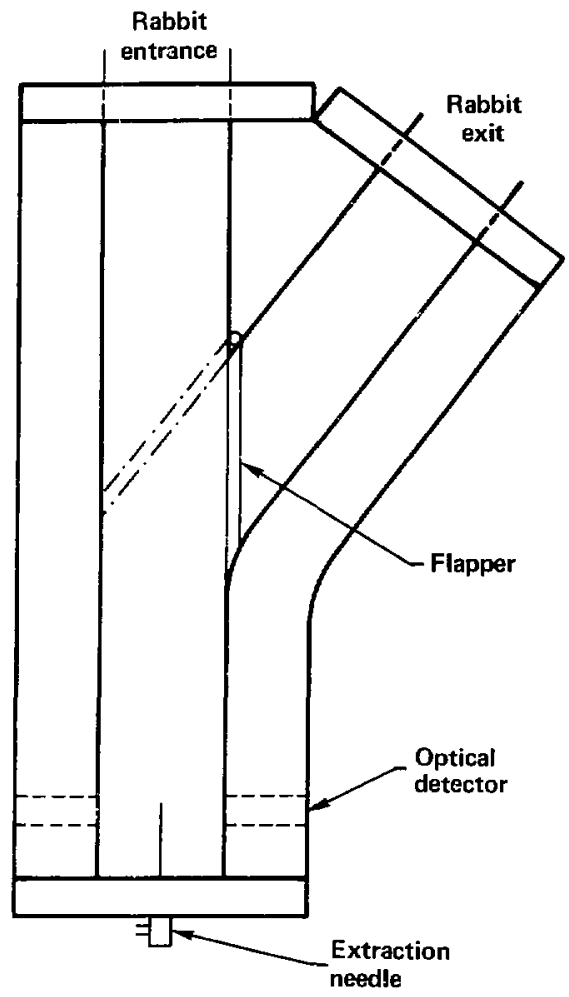

Fig. II.6. The receiver for AUTOBATCH. The rabbit enters the rertical channel and is ejected out the right channel as shown by arrows. The eject path is controlled by the flapper, which is moved to the position shown in dashed lines for ejection. The photo rell near the bottom of the receiver detects the presence of the rabbit. The extraction needle is shown in the bottom of the receiver.

The empty capsule was pneumatically ejected at the end of the chemistry sequence, with a flapper directing the capsule into the waste container (see Fig. 1I.6).

The major design consideration of these components was reliability without loss of speed. Because parts that must move rapidly often lack durability, I maintained a high overall cycle speed by allowing some of the transport functions to take place concurrently with other steps. For example, in the loader, ihis was achieved by loading the sample capsule while the previous sample was being processed at the chemistry station. In the receiver, the flapper moved and the empty capsule was ejected while the data acquisition was in progress. Only the diverter and the sample capsule itself had to move quickly. In Appendix 1, I give a detailed description of the components and compare the original components to the AUTOBATCH versions. 


\section{The Chemistry Subsystem}

To demonstrate the efficiency of the AUTOBATCH system, I selected a hydride chemistry to isolate the $5 \mathrm{~A}$ and $6 \mathrm{~A}$ elements. These regions have previously been inaccessible for detailed spectroscopic study. Both of these regions give very low yields with on-line isotope separators as shown in Fig. II.1, precluding their study by this technique. Trautmann et al., have measured halflives and major transitions of neutron rich As and Se isotopes (KRA75, KRA70) but their batch system is not suited for detailed spectroscopic examination of the short-lived isctopes required for nuclear structure studies (see the discussion on the Mainz baich system above).

The first step in my hydride chemistry is to produce the hydride gases of the elements by creating nascent hydrogen in aqueous solution. The hydride gases are then sequentially stripped from the gas stream with the desired element depositing in the sample cell. Elements which would also deposit are stripped out prior to the cell. Those which will not deposit in the sample cell are allowed to pass through.

The hydride reaction is achieved by $i$. strong reducing agent, sufficiently strong to reduce the hydrogen ion to nascent hydrogen. The elements of interest are also reduced from the positive valence states to the neutral state. Hydride gas formation dominates cver element formation for two reasons:

1. The conditions used form a large excess of nascent hydrogen compared to the element of interest, which statistically favors formation of the hydride gas.

2. The metal atoms are surrounded by a sphere of water molecules causing them to move slowly. The nascent hydrogen is not hydrated and can move very quickly.

The hydride gases can be produced by treating an acid solution of fission products with one of the following: (1) direct electrochemical production, (2) zinc metal powder, or (3) sodium borohydride solution. Electrochemical production of the hydride gases was ruled out since it required approximately $1000 \mathrm{~A}$ of current for $0.1 \mathrm{~s}$, beyond the capacity of available power supplies. Zinc metal powder, as used in the system at Mainz (FOL69), is traditionally considered the reagent of choice for this reaction. However, zinc metal is difficult to handle with automated equipment. The desire to automate the system dictated the choice of the sodium borohydride reaction. Sorlitm borohydride solution is easy to inject and easy to clean up in an automated a:'stem and gives sufficient yield of the desired hydrides to preserve sample purity.

In my hydride chemistry, the noble gases are first removed from the sample by sparging. The sample is acidified and thoroughly mixed and then the sodium borohydride is added and mixed. The gas phase containing the elements of interest and liquid phase containing the remaining fission products must then be separated. In a standard hydride distillation reaction, the slow step is separating the gas and liquid phases. Therefore, to use the hydride chemistry in the required time framo (less than a sccond), l har to devise some means of rapidly separating the two phases. Trautmann (TRA78) has noted th iny .. Nard chemical reactions, when performed in a standard way, are too slow for the $\mu_{d i}$ sses of .. chemistry. These reactions can, however, be used if they are performed in nons Jard ways that speed the reaction. To accomplish these steps very rapidly, 1 designed the still and cyclone separator shown in Fig. II.7.

The sample and reagents are introduced in to the still through ports that are tangential to the walls of the still. The tangential entry creates rapid rotational motion of the solutions, forcing them to remain on the walls of the still. This gives the large surface area required for good sparging and, at the same time, provides rapid and complete mixing of the reagents.

The gas and liquid phases are separated in a similar manner. The pressure created by the formation of the hydrogen gas (created in the still) drives the gas-liquid mixture into the cyclone separator. The tangential entry into the separator generates rapid rotational motion and the centrifugal force holds the liquid on the circular walls of the cyclone allowing the gas to escape out the center exit. In this manner, I achieved a very rapid gas-liquid separation.

I have made a film study of the cyclone separator (LIE79) showing that the cyclone performs as anticipated. she study made use of the high speed motion picture camera developed at LLNL, which was used at 2000 frames per second. At this speed the motion was slowed a factor of 100 times. This study showed that cyclonic motion was present in both the still and the separaior. In 


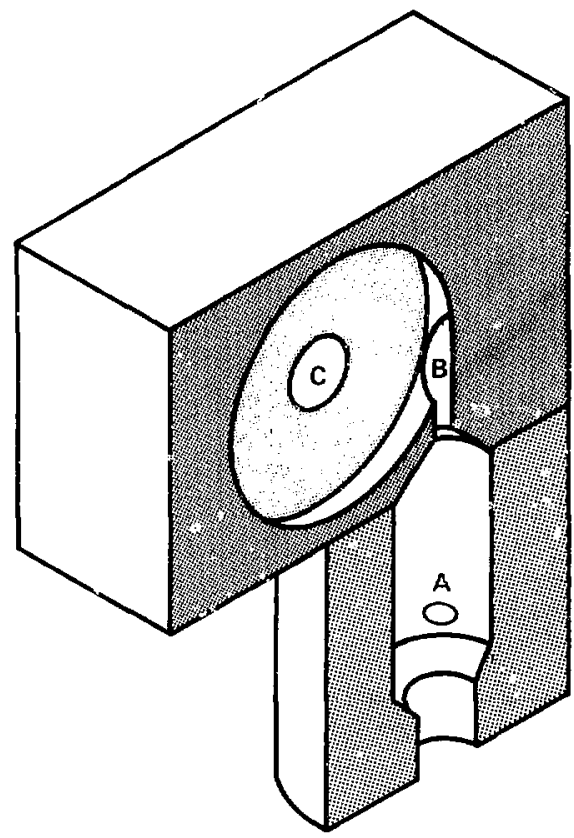

Fig. 11.7. The still and cyclone separator used in AUTOBATCH hydride chemistry. The still is the lower chamber, with a typical inlet port shown (A). The gas-liquid mixture is forced through orifice $B$ into the cyclone where the gas and liquid are separated. The gases exit through orifice $C$.

the still the motion produced effective mixing of the reagents. In the cyclone it effectively separated the gas and liquid phases.

In the standard hydride reaction, the gas and liquid phases are separated simply by gravity; this requires a large still and a gentle reaction. As a result, this step is very slow. An estimate of the separation enhancement possible with the cyclone separator is obtained from the flow rate of the gas-liquid mixture entering the cyclone:

$v_{1}=\frac{\text { volume }}{\text { area } \times \text { time }}$

$140 \mathrm{~cm} / \mathrm{s}=\frac{2 \mathrm{~m} !}{\pi \times 0.15^{2} \mathrm{~cm}^{2} \times 0.2 \mathrm{~s}}$,

where the sample volume is divided by the cross-sectional area of the orifice and the time required for the transfer. 1 ignore the effects of the expanding gas volume that actually serve to increase the velocity. The centrifugal acceleration is then

$\mathrm{a}=\frac{v_{\mathrm{i}}^{2}}{\mathrm{r}}=\frac{(140 \mathrm{~cm} / \mathrm{s})^{2}}{2 \mathrm{~cm} \times 980 \mathrm{~cm} / \mathrm{s}^{2}}=10 \mathrm{~g}$. 
Thus ! achieved a tenfold increase in the gas-liquid separation forces over that possible with the simple gravitational method.

This procedure generares the hydride homologues indicated previously. The next step is to isolate the desired elements from among these homologues.

The remaining chemical separation steps are carried out in the gas phase. The element of interest is collected in the sample cell on a glass-wool plug watted with the appropriate reagent. Those elements that would also collect on the glass wool wer? removed from the gas stream brfore they ieached the plug; the elements that would not collect were allowed to pass through.

\section{Fast Arsenic Chemistry}

Following the general chemistry procedure discussed above, the hydride chemistry specific for arsenic is as follows:

- A raw sample consisting of $1.0 \mathrm{mg}{ }^{235} \mathrm{U}$ dissolved in $0.7 \mathrm{ml}$ of $0.14 \mathrm{~N}$ sulfuric acid was irradiated for $150 \mathrm{~ms}$.

- The irradiated sample is extracted and sparged for $500 \mathrm{~ms}$ to remove the noble gases.

- The sparged sample is then acidified with $1 \mathrm{ml}$ of $9 \mathrm{M} \mathrm{HCl}$ containing $2 \mathrm{mg} / \mathrm{ml} \mathrm{Sn^{2+ }}$ as a hold-back carrier, to reduce the tin contamination.

- $2 \mathrm{ml}$ of sodium borohydrite solution $(125 \mathrm{~g} / \mathrm{l} \mathrm{NaBH})$ is added.

- The hydride gases are passed through a trap to remove entrained water, $\mathrm{SbH}_{3}, \mathrm{H}_{2} \mathrm{Se}$, $\mathrm{H}_{2} \mathrm{Tc}$, and $\mathrm{SnH}_{4}$. This trap is clear plastic tube $1.8 \mathrm{~cm}$ in diameter and is filled, in the direction of gas flow, with $15 \mathrm{~cm}$ of anhydrous $\mathrm{CaSO}_{4}$ (Drierite), $40 \mathrm{~cm}$ of anhydrous sodium hydroxide (Ascarite) followed by $5 \mathrm{~cm}$ of anhydrous $\mathrm{CaSO}_{4}$ (Drierite).

- The arsenic is collected on a glass-wool plug wetted with $9 \mathrm{M} \mathrm{HCl}$ containing $5 \mathrm{ml} / 1$ liquid bromine.

Table II.1 gives the computer-controlled timing sequence for the chemistry to separate ${ }^{R-4} \mathrm{As}$. Figure II.8 shows the chemistry subsystem and its control valves. Figure 11.9 shows the flow of the elements through the system.

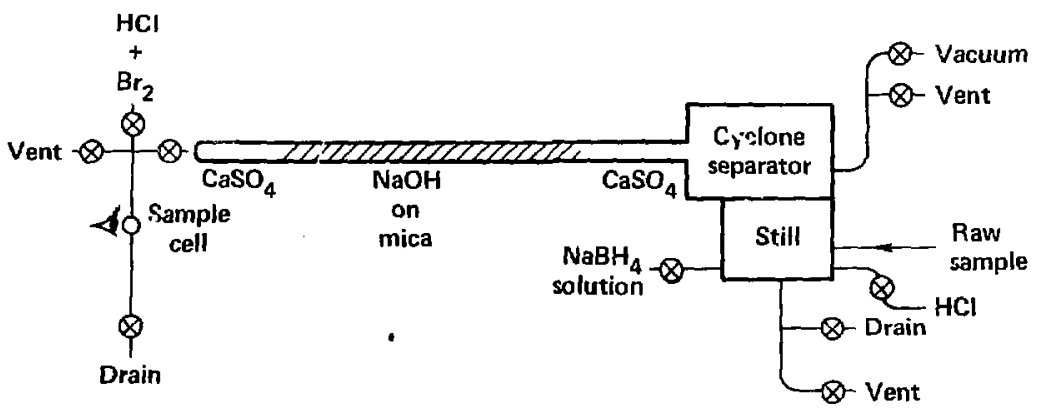

Fig II.B. The AUTOBATCH chemistry system is shown schematically. The are computer controlled solenoid valves. A gamma ray detector is shown to the left of the sample cell. Flow direction is indicated by arrows except for the vents which are bi-directional. 


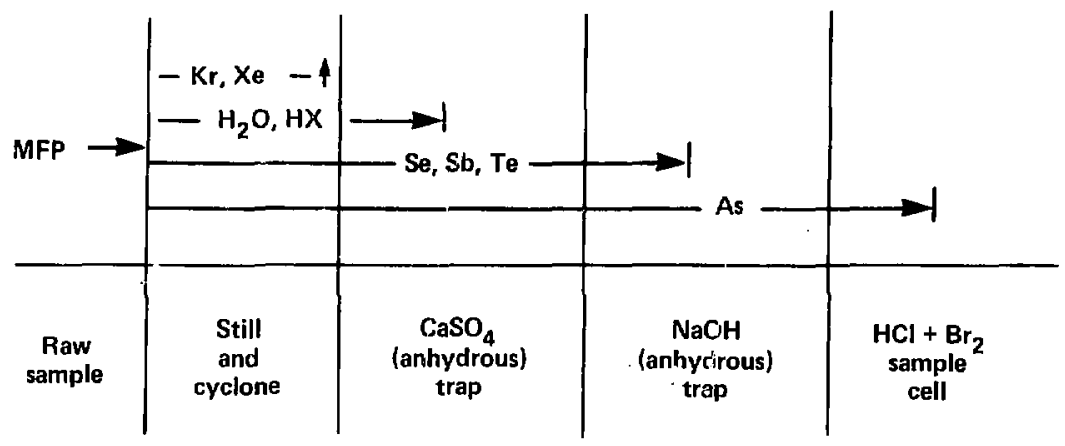

Fig. II.9. The flow of various compounds in the hydride chemistry. Indicated compounds go to the end of the bar except for $\mathrm{Kr}$ and $\mathrm{Xe}$ which are removed before reaching the $\mathrm{CaSO}_{4}$ trap. MFP means mixed fission products and $\mathrm{HX}$ means $\mathrm{HCl}, \mathrm{HBr}$ and $\mathrm{HI}$.

Table II.1. Computer-controlled timing sequence for ${ }^{84} \mathrm{As}$ (5-s half-life).

\begin{tabular}{|c|c|c|}
\hline Operation & $\begin{array}{l}\text { Step } \\
\text { time, } s\end{array}$ & $\begin{array}{l}\text { Elapsed } \\
\text { time, } 5\end{array}$ \\
\hline Return sample from reactor core & 1.0 & 1.00 \\
\hline $\begin{array}{l}\text { Extract sample from capsule and purge } \\
\text { noble-gas fission products }\end{array}$ & 0.5 & 1.50 \\
\hline Acidify sample with $\mathrm{HCl}+\mathrm{Sn}(1 \mathrm{ml})^{\mathrm{b}}$ & 0.09 & $\mathbf{t . 5 9}$ \\
\hline Inject $\mathrm{NaBH}_{4}$, solution into still $(2 \mathrm{ml})$ & 0.1 & 1.69 \\
\hline $\begin{array}{l}\text { Inject gas-liquid mixture into cyclone } \\
\text { and pass hydride gas through trap }\end{array}$ & 0.25 & 1.94 \\
\hline Start detectors counting & - & 1.94 \\
\hline Drain still and cyclone & 1.6 & 3.54 \\
\hline Wash still and cylone with water & 0.6 & 4.14 \\
\hline Drain still and cyclone & 1.0 & 5.14 \\
\hline Purge $\mathrm{NaBH}_{4}$ line & 0.9 & 6.04 \\
\hline Rinse still with water & 1.2 & 7.24 \\
\hline Drain still & 1.2 & 8.44 \\
\hline Recondition still with $\mathrm{HCl}+\mathrm{Sn}_{n}$ & 0.24 & 8.68 \\
\hline Drain still & 2.4 & 11.08 \\
\hline Wait for end of data-arquisition step ${ }^{c}$ & $\mathbf{T}$ & $11.08+\mathrm{T}$ \\
\hline Wash sample cell with $\mathrm{HCl}+\mathrm{Br}$ & 3.1 & $14.18+T$ \\
\hline Remove excess $\mathrm{HCl}+\mathrm{Br}$ from sample cell & 2.4 & $16.58+T$ \\
\hline Total chemistry time & & $16.53+T$ \\
\hline
\end{tabular}

- All volumes meastired by time valve were open; volumes given are estimates.

"A t0-5 delay was added here for ${ }^{\mathrm{B2}, \mathrm{n3}}$ As studies.

'Additional time may be required for data aquisition. 


\section{Experimental Measurements}

With the fast chemistry system described in the pievious section, I made detailed gamma-ray spectroscopic measurements following the $\beta$ decay of fission product (FP) arsenic, with emphasis on the decay of ${ }^{4.3}$ As to study the nuclear structure of ${ }^{83} \mathrm{Se}$. This work allowed me to make the first identification of the intruder vibrational band structure in a 49-neutron nucleus. In the process, I deduced that ${ }^{\mathrm{B} .3} \mathrm{As}$ has a ground state spin and parity of $5 / 2^{-}$and redetermined the absolute ground state $\beta$ branch from ${ }^{83} \mathrm{Se}^{\mathrm{m}}$ to be $38 \%$.

l carried out four separate experiments on the FP arsenic decay. These experiments studied ${ }^{8-1}$ As decay and ${ }^{82,83}$ As decay, determined the energy calibration, and investigated growth and decay of daughter species. The growth and decay experiment was done to establish the $\beta$ branches from ${ }^{\mathrm{k4}} \mathrm{As}$ ground state to ${ }^{84} \mathrm{Se}$ the ground state and from the ${ }^{\mathrm{K3}} \mathrm{As}$ ground state to the ${ }^{\mathrm{R}} \mathrm{S} \mathrm{Se}$ isomeric state. The parameters pertinent to these measurements are given in Tables III.1a and III.1b. For the ${ }^{82, \mathrm{~B} 3}$ As decay studies, I added a 10-s delay before separating the arsenic from the fission products to allow the 5-S ${ }^{84}$ As to decay, thus greatly reducing both the ${ }^{84} \mathrm{As}$ and ${ }^{84} \mathrm{Se}$ activities in the counted samples (see Table II.1).

\section{Spectroscopy Apparatus}

Since the sample counting cell was an integral part of the chemistry system, the detectors were immediately adjacent to the chemistry facility. This, in turn, necessitated that the detectors be heavily shielded from the residual fission-product activity. To maximize use of each sample, simultaneous single-parameter gamma-ray and three-parameter gamma-gamma-time coincidence measurements were made. The detector-sample cell arrangement is shown in Fig. III.1. The shielding consisted of 15 to $25 \mathrm{~cm}$ of lead berween the detertors' active volumes and external sources of activity. The arrangement shown was used for the coincidence detectors at $180^{\circ}$. The sample cell was $0.5 \mathrm{~cm}$ in diameter and placed between two $0.5-\mathrm{cm}$-thick lead shields with columnating holes between the detertors and the sample cell. The shields reduced detector-todetector backscattering of photons, which would give intense false coincidences. The shields were carefully arranged $: 0$ that they did not partially screen the singles detector. The remainder of the data acquisition system was located in a separate room $12 \mathrm{~m}$ distant from the detectors.

The details of the spectroscopy are given in Ref. LAN79. Here, I give a brief description of the spectroscopy apparatus emphasizing the improvements made since that report.

\section{Gamma-Ray Singles Measurements}

Single-parameter MCMS data were acquired to establish correct energies and intensities. Most of the singles data were acquired using a Data General In', NOVA 1200 minicomputer as a 4096-channel analyzer which was capable of holding six 4096 'annel spectra. For the ${ }^{\mathrm{B} 4} \mathrm{As}$ experiment, some of the singles data were taken on a Canberra 80 analyzer using 8192 channels and two MCMS spectra to obtain good energy resolution over an $8-\mathrm{MeV}$ range.

The data for the growth and decay experiment were acquired using a real-time clock. Dead time was monitored with the $392-\mathrm{keV}$ gamma ray from a ${ }^{113} \mathrm{~S}$ radioactive source. The observed count rate for this photopeak measured the relative dead time of the counting system. Tin-113 was chosen because it emits gamma rays only at an energy below that of the gamma rays of interest.

An ORTEC high-purity germanium (HPGe) detector was used for all single-parameter data. Its efficiency was calibrated from 0.1 to $10 \mathrm{MeV}$ (LIN80). This calibration included the cadmiumcopper cap (each $0.7 \mathrm{~mm}$ thick) on the detector, which was used to absorb the fluorescent lead $x$-rays. This detector had a FWHM of $2.2 \mathrm{keV}$ at the ${ }^{60} \mathrm{Co} 1332-\mathrm{keV}$ gamma ray. The FWHM observed in the data was $3.0 \mathrm{keV}$ at $1331 \mathrm{keV}$ due to count rate variation during the data acquisition period. 
Table III.1a. Details of the gamma-ray spectroscopy experiments for ${ }^{22,83,84} A s \beta^{-}$decay, giving timing, energy range, gain, and peak to background ratios foi sample transitions. The two ${ }^{84}$ As experiments differ in the timing and energy ranges ustd.

\begin{tabular}{|c|c|c|c|c|}
\hline & \multicolumn{4}{|c|}{ SINGLES EXPERIMENT } \\
\hline & ${ }^{82,83} \mathrm{As}$ & ${ }^{-A s-H i}$ & As As & Calibration \\
\hline \multicolumn{5}{|l|}{ Timing (s) } \\
\hline Start separation & 11 & 1 & $\mathbf{1}$ & 1 \\
\hline \multirow[t]{4}{*}{ Count time } & $12.0-26.7$ & $2-7$ & $2.0-4.1$ & $2.0-8.6$ \\
\hline & $26.7-40.6$ & $9-13$ & 4.1-6.2 & $8.6-16.1$ \\
\hline & $40.6-54.2$ & & $6.2-8.3$ & \\
\hline & & & $8.3-13.4$ & \\
\hline Gross counts & 7.4E8 & $1.0 \mathrm{E} 8$ & $4.1 \mathrm{E7}$ & $9.3 \mathrm{E} 7$ \\
\hline \multirow[t]{3}{*}{ in spectrum } & $3.8 E 8$ & $4.6 \mathrm{E}^{\prime}$ & $3.2 \mathrm{E} 7$ & $6.4 \mathrm{E} 7$ \\
\hline & 2.3E8 & & 2.6E7 & \\
\hline & & & 4.7E7 & \\
\hline Enargy range (MeV) & $0-4.1$ & $0.54-8.5$ & $0-4.1$ & $0-4.1$ \\
\hline Gain (keV/chan.) & 1.0 & 1.08 & 1.0 & 1.0 \\
\hline \multicolumn{5}{|l|}{ Peak/background } \\
\hline Peak & 734-keV & 1454-keV & 1454-keV & \\
\hline Counts & 2.2E6/2.1E5 & 2.1E6/1.6E5 & $3.1 \mathrm{E} 5 / 2.5 \mathrm{E} 4$ & \\
\hline
\end{tabular}

Table III.1b. Details of the coincidence gamma-ray spectroscopy experiments following the decay of ${ }^{82,83,84} \mathrm{As}$. Information is the same as in Table III.a.

\begin{tabular}{|c|c|c|c|}
\hline & \multicolumn{3}{|c|}{ COINCIDENCE EXPERIMERTTS } \\
\hline & ${ }^{82,83} \mathrm{As}$ & $\begin{array}{c}{ }^{203} \mathrm{As} \\
\text { angular } \\
\text { correlation }\end{array}$ & ${ }^{84}$ As \\
\hline \multicolumn{4}{|l|}{ Timing (s) } \\
\hline Start separation & 11 & 11 & 1 \\
\hline Start count & 12 & 12 & 2 \\
\hline End count & 55 & 55 & 15 \\
\hline Detector configuration & $180^{\circ}$ & $99^{\circ} \& 180^{\circ}$ & $180^{\circ}$ \\
\hline Energy range (MeV) & 0.6 .4 & $0-6.4$ & $0-8.2$ \\
\hline Gain (keV/chan.) & 0.83 & 0.83 & 1.0 \\
\hline \multicolumn{4}{|l|}{ Peak/Background } \\
\hline Slice & 734-keV & 654-keV & 1454-keV \\
\hline $\begin{array}{l}\text { Peak } \\
\text { Counts }\end{array}$ & $\begin{array}{l}\text { 1113-keV } \\
24400 / 1000\end{array}$ & $\begin{array}{l}755-k e V \\
1450 / 300 \text { at } 90^{\circ}\end{array}$ & $\begin{array}{l}\text { 667-keV } \\
12500 / 1400\end{array}$ \\
\hline $\begin{array}{l}\text { Peak } \\
\text { Counts }\end{array}$ & $\begin{array}{l}2729-k e V \\
295 / 50\end{array}$ & $\begin{array}{l}755-k e V \\
2750 / 450 \text { at } 180^{\circ}\end{array}$ & $\begin{array}{l}\text { 5151-keV } \\
110 / 40\end{array}$ \\
\hline
\end{tabular}

For the energy calibration, I used ${ }^{133} \mathrm{Ba},{ }^{134} \mathrm{Cs}$, and ${ }^{56} \mathrm{Co}$ as standards (MEY78) to span the energy region from 0 to $3.5 \mathrm{MeV}$. Above $3.5 \mathrm{MeV}$, I used the gamma rays from the Cdin,g) reaction for energy calibration (GRO68). The source of neutrons was beta-delayed neutrons from fission products. These standards gave a reliable energy calibration over the energy range fror 0.2 to $7.0 \mathrm{MeV}$. 


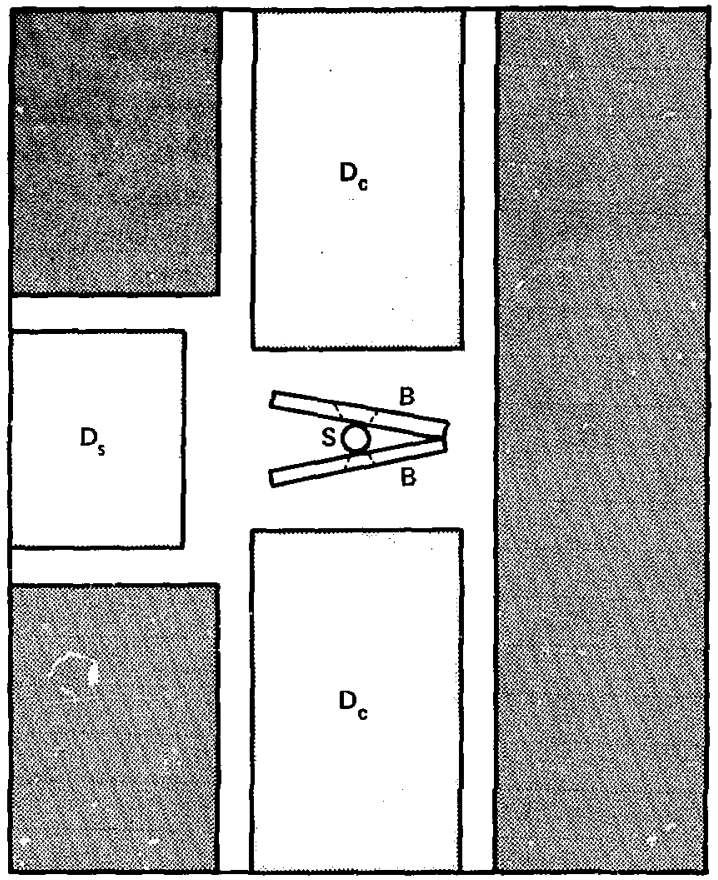

Fig. III.1. Top view of the detector arrangement. The coincidence detectors, $D_{c^{\prime}}$ are $180^{\circ}$ apart. The singles detector is labeled $D_{s}$, ihe sample cell is labeled $S$. The backscatter shielding, B, is shown with the collimating holes drawn in dotted lines.

\section{Gamma-Ray Coincidence Measurements}

The coincidence measurements recorded the energy of two gamma rays and the time separating them, provided the time separating them was less than $350 \mathrm{~ns}$. This defined a coincidence event. The detector charge collection time and other electronic noise produced a time-toamplitude conversion (TAC) resolution of $10 \mathrm{~ns}$ FWHM. The detectors used to acquire the coincidence data were high-resolution ORTEC Ge(Li) detectors. The energy resolution of the detector was $2.2 \mathrm{keV}$ FWHM for the ${ }^{610} \mathrm{Co} 1332-\mathrm{keV}$ gamma ray.

All excited levels can decay by gamma-ray emission with half-lives ranging upward from less than $1 \mathrm{fs}$. The $\mathrm{Ge}(\mathrm{Li})$ detector system allows measurement of isomeric half-lives down to approximately 2 ns. To retain this resolution, I set the TAC gain to 1.39 ns per channel. Over the 512 channels available, this gave $350 \mathrm{~ns}$ over which a delay could be measured. Experimentally, the limits on observable level half-lives could be crudely determined in that I couid not chserve the half-life of either the $654-\mathrm{keV}$ level in ${ }^{\mathrm{S} e}\left(13.4 \mathrm{ps}\right.$ ) or the $1225 \mathrm{keV}$ level in ${ }^{131} \mathrm{Sn}(150 \mu \mathrm{s})$, although there was sufficient singles intensity of the pertinent gamma rays to easily see the halflives if they were not outside my experimental limits.

The TAC data was used also to correct for chance coincidence events. A chance event occurred when the two gamma rays causing the coincidence event did not originate in the same 
atorn. I assumed that the TAC profile spectrum consisted of a Gaussian peak of true coincidence events on a flat background of chance events. I then accepted all events with a TAC value within the FWTM of the true coincidence peak and subtracterl the events in an equal number of channels well removed from the true coincidence peak.

Two different energy ranges were used to cover the different possible ranges of excitation energy in the selenium nuclei. These ranges were set to observe the most energetic gamma ray possible in the nuclei of interest, which is limited by the $Q_{B^{-}}$value for the beta decays of the different arsenic species. The $\mathrm{Q}_{B^{-}}$values are $6.4 \mathrm{MeV}$ for ${ }^{82} \mathrm{As}, 5.6 \mathrm{MeV}$ for ${ }^{83} \mathrm{As}$, and $9.8 \mathrm{MeV}$ for ${ }^{84} \mathrm{As}$. However, the upper limit for ${ }^{84} \mathrm{As}$ is further reduced to the neutron binding energy in ${ }^{84} \mathrm{Se}$, $8.4 \mathrm{MeV}$. Levels populated above the neutron binding energy decay primarily by neutron emission and do not contribute significantly to the gamma-ray spectrum. With the upper energy limit set, the lower energy limit is set by the dynamic range of the analog-to-digital converter. The dynamic range is a factor of approximately 35, giving a lower energy limit of $180 \mathrm{keV}$ for ${ }^{\mathrm{B}} \mathrm{As}$.

Included in the ${ }^{82,{ }^{3}} \mathrm{As}$ measurements were angular correlation measurements with the coincidence detectors at $90^{\circ}$ and $180^{\circ}$. These measurements were made to establish the $0^{+} \rightarrow 2^{+}-$ $0{ }^{4} \gamma$ cascades in ${ }^{82} \mathrm{Se}$. They were also used to measure the anisotropy when the coincidence data were used to determine transition intensity. The detectors were moved to a distance of $10 \mathrm{~cm}$ for the angular correlation measurements. At this distance a detector presented an area of 0.16 steradian (an included angle of $9^{\circ}$ ) to the decaying source.

Shown in Fig. IIl. 2 is plot of the spectrum of events in coincidence with the $1455-\mathrm{keV}$ gamma ray obtained from the ${ }^{84}$ As experiment. These coincidences occur primarily in the ${ }^{84}$ Se nucleus. The total number of counts in the $667-\mathrm{keV}$ and $5151-\mathrm{keV}$ peaks from the $1454-\mathrm{keV}$ gate is an indicator of the quality of the experiment and the validity of my assignments for high-lying levels. For ${ }^{\text {B }} \mathrm{As}$, I include a plot of the $582 \mathrm{keV}$ gate (Fig. III.3) with an inset showing the low-intensity transitions from the natural parity levels. The $582-\mathrm{keV}$ gamma ray has an intensity of 1095 relative to 1000 for the $734-\mathrm{keV}$ gamma ray and the $381-\mathrm{keV}$ gamma ray has an intensity of 0.4 showing that very weak transitions can be measured.

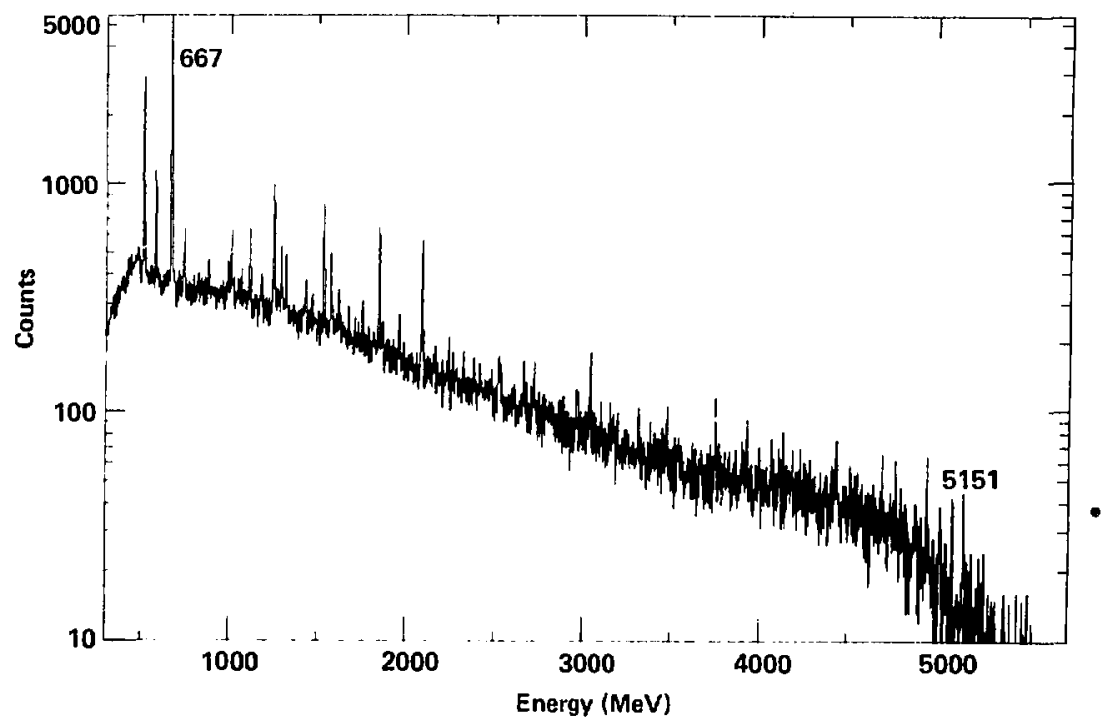

Fig. III.2. Plot of the $1455-k e V$ gate from the ${ }^{84}$ As experiment. 


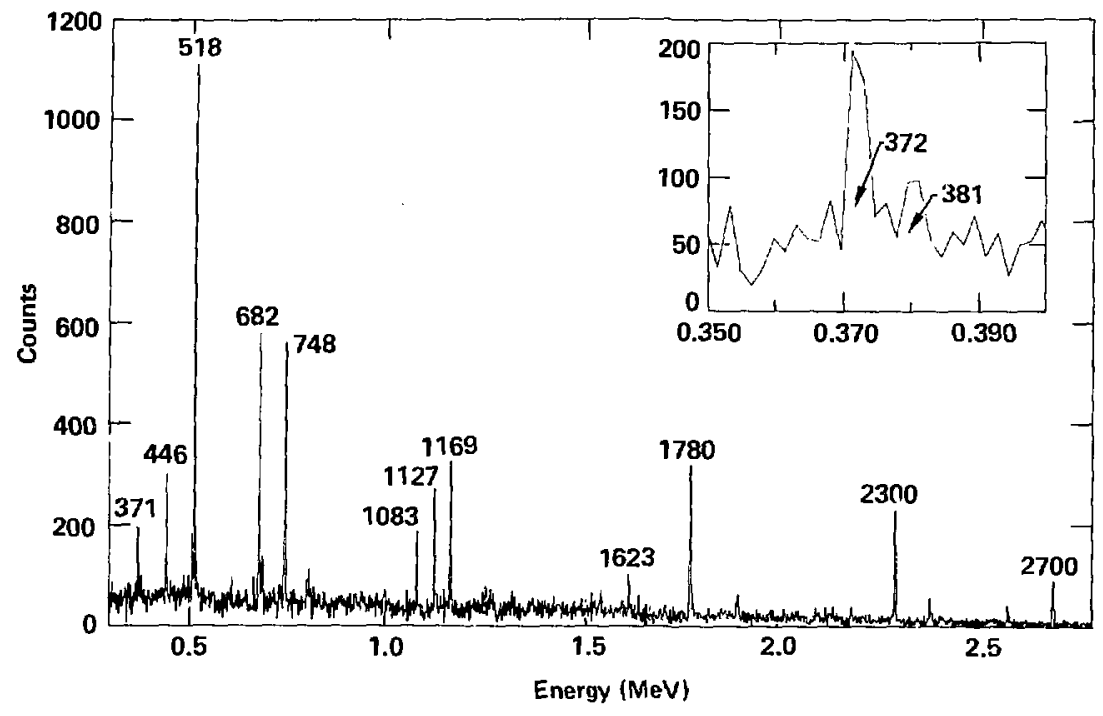

Fig. III.3. Plot of the $582-\mathrm{keV}$ gate from the ${ }^{82,83}$ As experiment. The inset shows the low intensity transition from the $3 / 2_{1}^{-}$state to the $5 / 2_{1}^{+}$state.

\section{Data Analysis}

Single-parameter data were analyzed with either the GAMANAL (GUN72) or FITEK (STO80) computer codes; the use of GAMANAL is described in Ref. LAN79. However, the analysis of my spectra by GAMANAL improved remarkably when the peak shape parameter P5 was reduced from 1.2 to 0.76 . This parameter is not usually changed. Its effect is to increase the tail width of the peak. Coincidence data were first sliced (LAN79) to obtain single-parameter data from threeparameter data, then analyzed with the computer code PK2D (described below).

FITEK is an interactive routine, which uses peak shape parameters similar to those of GAMANAL. The user defines the fit region, the background at the endpoints of the fit region, and the peaks and their crude location by cursor conirol. The parameters may be constrained or allowed to vary until the best least-sqliares fit to a peak is obtained. The user also sets the hackground step height as a fraction of the peak height. In the fitting process, peaks may be dropped but not added. Once the best fit is obtained, the fit region is displayed showing the both experimental and calculated spectra and the background. The interactive nature of FITEK allows the user to inspect possible solutions much more easily than with GAMANAL, but correspondingly is much slowe1.

In the coincidence spectra generally poor statistics precluded the use of either GAMANAL or FITEK, which require good statistics. However, having produced 600 gated spectra slices from the two coincidence experiments, an automated procedure for analyzing the energy and intensity in the coincidence was needed. The common procedure for peak analysis in coincidence data is: first, to find a peak, second, find its endpoints, next, sum the counts over the peak, and then, subtract the background. This procedure cannot be automated because of the poor statistics of most coincidence spectra. Stevenson (STE77) had used an algorithm that took $p$ channels of peak and $b$ channels of background on each side of the peak and at each channel $k$ defined 


$$
\left.Y_{k}=\sum_{p}^{-\cdot} \mid C_{r}\right]-\frac{p}{2 b} \sum_{2 p}\left[C_{1}\right]
$$

This algorithm generates reasonable peak areas but poor peak centroids. This is because the algorithm peak width is discrete but the experimental peak width varies smoothly as a function of energy.

A continuous function that imitates this algorithm is ti.e second derivative of a Gaussian, It can be easily shown that convoluting this function with n Gaussian, constant, linear, and quadratic function gives the secund derivati e of a Gaussian, zero, zero and constant results, respectively. The results ior the last three funcrions show that convoluting a spectrum background with the second derivative of a Gaussian should give nearly zero since, in principle, the background is smooth. For a convoluted Caussian peak, the amplitude diffews only by a multiplicative constant from the original peak amplitude and the centroid is unchanged when compared to the original peak. This suggests that, even though the peak shapes are not Gaussian, this algorithm would return reliable energies and intensities. Testing siowed that this algorithm performed nearly as desired. It has two limitations: (1) the requirement of prior knowledge of peak widths and (2) the isck of noise damping. For the returned ampliiude to be the peak area, the convolutirn function must be tite same width as the peak. The peak widths are easily determined using the standard function of energy (CUN72). Noise is not damped because the convolution function passes a sine wave with a wave length comparable to the convolution width.

The convolution function used is

$$
\left.D(m)=\left\{1-2(n / s)^{2}\right\} \exp (-\mid n / s\}^{2}\right\}
$$

where $s$ is the width parameter and $n=C-C^{\prime}$, where $C$ is the center of the convolution function and $C^{\prime}$ is the channel being considered. When ronvoluted with a Gaussian of area $A_{0}$ and width $p$, this results in

$$
\gamma C l=\Lambda_{0} \frac{s^{2}}{s^{2}+r^{2}}\left(1-2 \frac{C^{2}}{s^{2}+\mu^{2}}\right) \exp \left(-\frac{C^{2}}{s^{2}+p^{2}}\right) .
$$

The actual convolution is carried out as the sum. In channel notation

$Y_{1}=C_{1}+\underset{n}{\vdots_{1}}\left[\left(C_{1}+C_{1}, \ldots\right] \times[m]\right.$,

where $C_{-}$are the contents of channel $i$. The photopeak area and position are determined by fitting a parabola to the three points at every local maxima in the convoluted spectrum. This routine determines peak area and centroids without fitting the peaks. For coincidence spectra this is a distinct improvement over previous procedures.

\section{Half-Life of the 582-keV Level}

The half-life of the 582-keV level had not been measured, but while I was analyzing the data Hoff (HOF81) reported a tentative measurement of $5.0 \mathrm{~ns}$ based on the ${ }^{82} \mathrm{Se}(d, p)^{83}$ Se reaction. I determined this half-life from my gamma-gamma-time coincidence data. As will be discussed later, this half-life is important for the intruder levels, which are expected to have strongly hindered transitions to the natural levels. 
The timing spectra were generated by gating on the energies of the tw'n coincident gamma rays to create the spectrum of timing values for that coincidence. The TAC peak should have the shape of an exponential half-life curve smeared by a Gaussian:

$Y(t)=\int_{1}^{\prime} A_{0} \exp \left(-\lambda \| t+t^{\prime}\right) \mid \frac{\exp \left(-\left(t^{\prime} / s^{2}\right)\right.}{s, \pi} d I^{\prime}$

where $t$ is the time, $t^{\prime}$ is the dummy variable for integration, $A_{0}$ is the height of the exponential curve, $\lambda$ is the decay constant, and $s$ is the Gaussian width which describes the timing resolution of the dctector and electronics. If there is a significant amount of data in the region $1 \gg s$ (e.g. $t>3 s$ ), then the lower limit can be replaced by infinity and

$Y\left(f^{\prime}=A_{0} \exp (-\lambda l) \exp \left((\lambda s / 2)^{2}\right)\right.$

is obtained, where $\lambda$ is the logarithmic slope of $Y$. In this case $s=6$ and there was very little data in the region where $t$ is large; the entire peak must be fit. In working with the ciata, it was converient to work in channels rather than time. The half-life was the tail width, In $2 / \lambda$ wl:en fitting the time peak with Eq. (1II.5).

To accurately determine the half-life when it is approximately equal to the timing resolution of the detector and electronics, I had to fit the entire timing spectrum. The timing resolution was determined from the timing peak resulting from the coincidence of the 734 - and $1113-\mathrm{keV}$ gamma-rays which is believed to have a half-life less than $0.1 \mathrm{~ns}$. This peak was fit first with the tail parameters forced to zero, giving a FWHM $=9.76 \pm 0.08$ channels. Freeing the tail parameters changed the FWHM to $9.50 \pm 0.14$ channels with no observable improvement in the fit. These analyses showed that the peak was nearly symmetric, hence reasonable for fitting the peak width. However, both FWHM values produce a peak wider than the experimental peak at the extremes, where the tail width is most strongly affected. I determined that the FWHM $=9.4$ gave a better fit in the outer portions of the peak and used this width in analyzing the $582-\mathrm{keV}$ level time spectrum. Comparisons of calculation and experiment for both widths are shown in Fig. III.4.

The time spectrum used for the $582-\mathrm{keV}$ level was a sum of direct coincidences with the 582$\mathrm{keV}$ transition (the 518-, 682-, 748-, 1126-, 2300-, 2700-keV coincidences with the 582-keV transition were used). Before fitting the $582-\mathrm{keV}$ level time spectrum, it was evaluated with FITEK. With the tail parameters forced to zero, the peak had distinctly asymmetric residuals. This residual asymmetry was removed when the tailing parameters were freed. The FITEK tail width was measured to be $2.5 \pm 0.1$ channel with a FWHM $=9.75$. These values were not used directly because of uncertainty of the effect of the tail truncation device used in FITEK on the tail width.

1 then calculated spectra from Eq. (III.5) for various tail widths and compared them to the experimental spectra. The best fit to the $582-\mathrm{keV}$ level time spectrum was obtained with a tail width of 2.2 channels as shown in Fig. III.5a using a FWHM of 9.4 channels. For comparison, the experimental spectrum is also compared to calculated spectra with 2.0- and 2.4-channei tail widths in Fig. III.5b. The uncertainty in the FWHM is taken as 0.4 channel, the difference between the FITEK value and the FWHM used, and the uncertainty in the tail width is 0.2 channel, estimated from inspection of Figs. III.5a and III.5b. Treating these as independent errors, the total error is 0.45 channel. The gain in the TAC spectrum was $1.39 \mathrm{~ns} / \mathrm{ch}$ annel; thus, the half-life was determined to be $3.1 \pm 0.6 \mathrm{~ns}$.

I have received information from Hoff (HOF82) that on remeasurement he obtained a value of $3.5 \pm 0.2 \mathrm{~ns}$. 


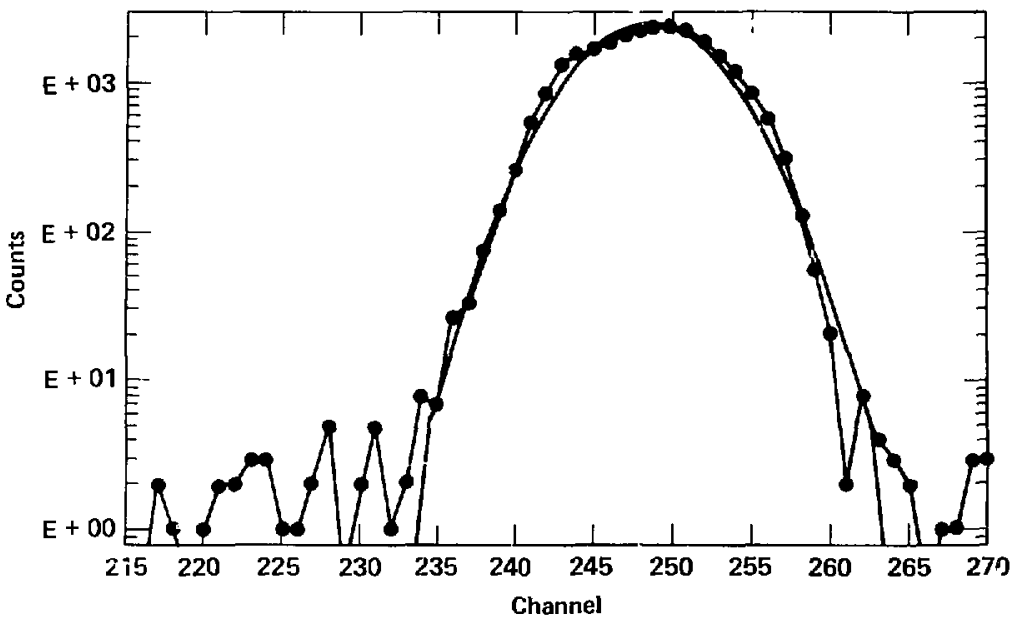

Fig. III.4. Comparison of the 734-1113-keV coincidence timing spectrum to a spectrum calculated with a 9.40 channel timing resolution. $(-=$ data, $-=$ calculation.)

23 


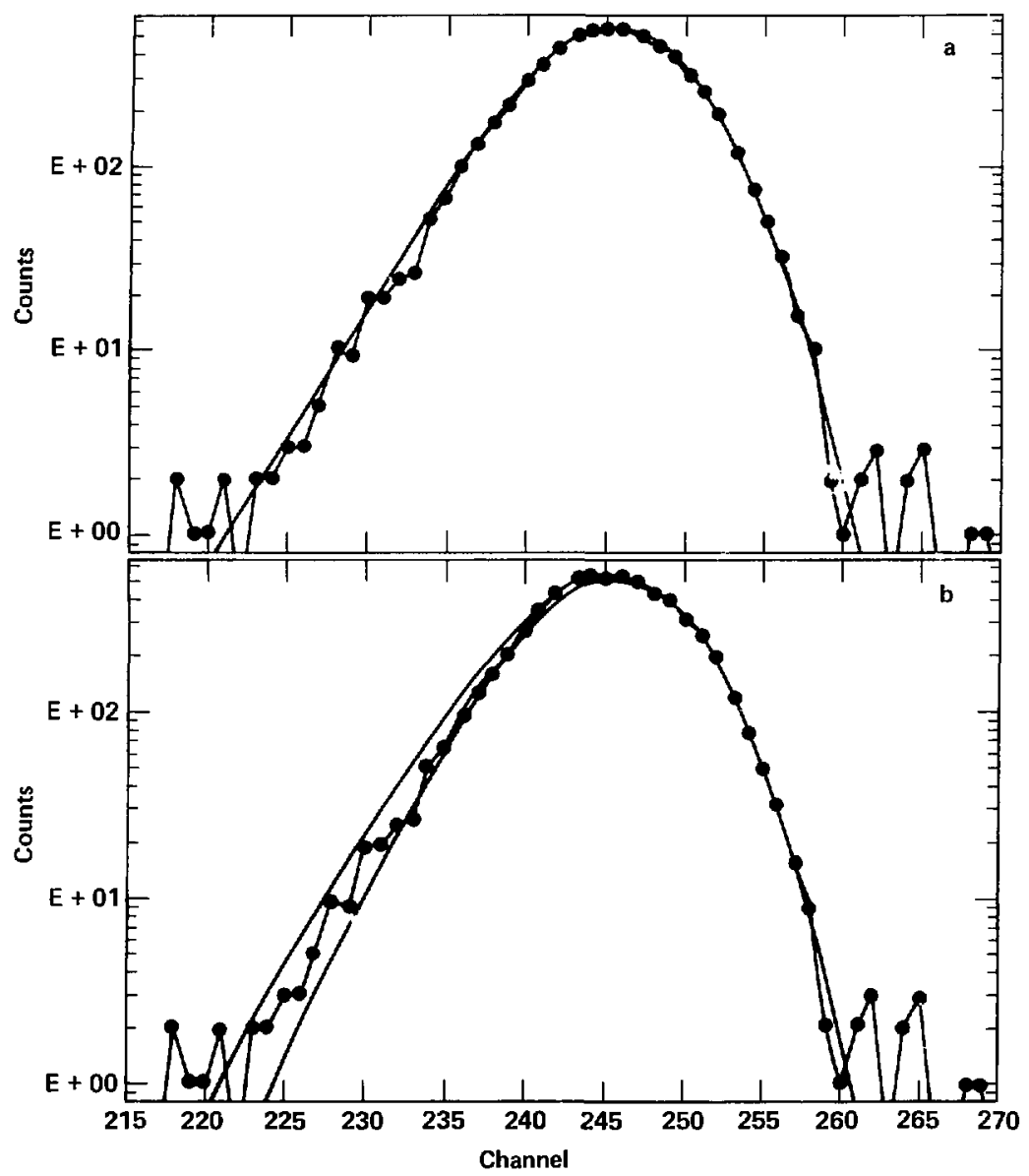

Fig. III.5. Comparison of the 582-keV level TAC spectrum with spectra calculated with a) 2.2 and b) 2.0 and 2.4 channel half-lives. ( $=$ data, $-=$ calculation).

\section{Data Analysis and Decay Scheme}

The decay scheme for ${ }^{83}$ As was developed primarily from the coincidence data. The singles data were used to establish energies and intensities, frequently with the aid of the coincidence data. The decay scheme arguments are presented in three Tables: IV.1, the coincidences observed for ${ }^{83} \mathrm{As}$ decay; IV.2, level justification; and IV.3, transition placement. The growth and decay experiment is evaluated to determine the $\beta^{-}$intensity to the ${ }^{83} \mathrm{Se}^{\mathrm{m}}$ state, which gives the spin and parity of the ${ }^{83} A_{5}$ ground state. The arguments for assigning spin and parity are given. 
Table IV.1. Coincidences used in constructing the ${ }^{83}$ As decay scheme. Tabulated are the coincidence spectra (Gate) and the peaks therein labeled by their energies in keV. The notes are at the end of the table, indicating the nuclide(s) to which the coincidence is assigned. The question marks indicate either that the coincidence was not assigned to any decay or that it is assigned hut not placed in the decay scheme.

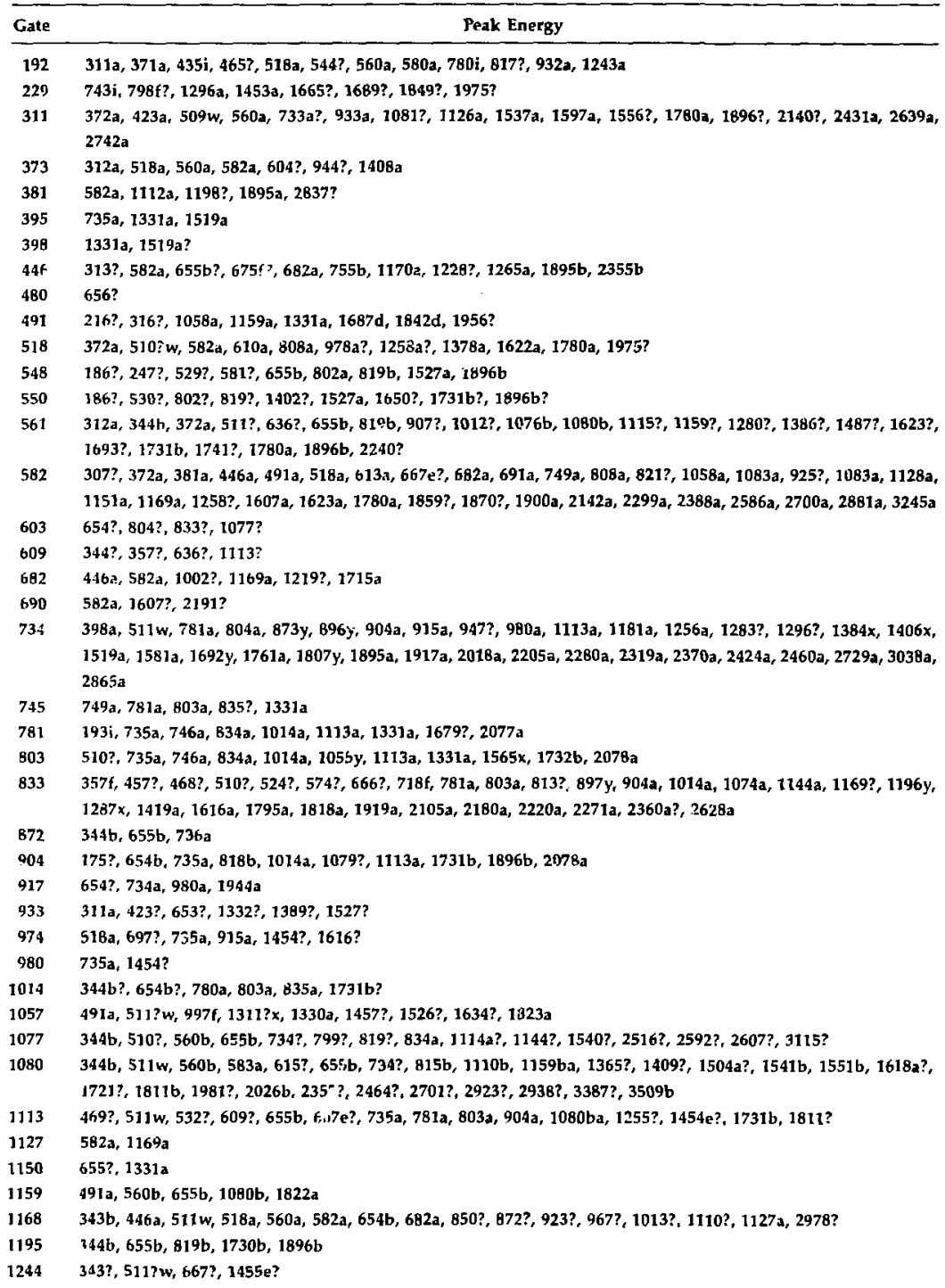


Table IV.1. (Continued.)

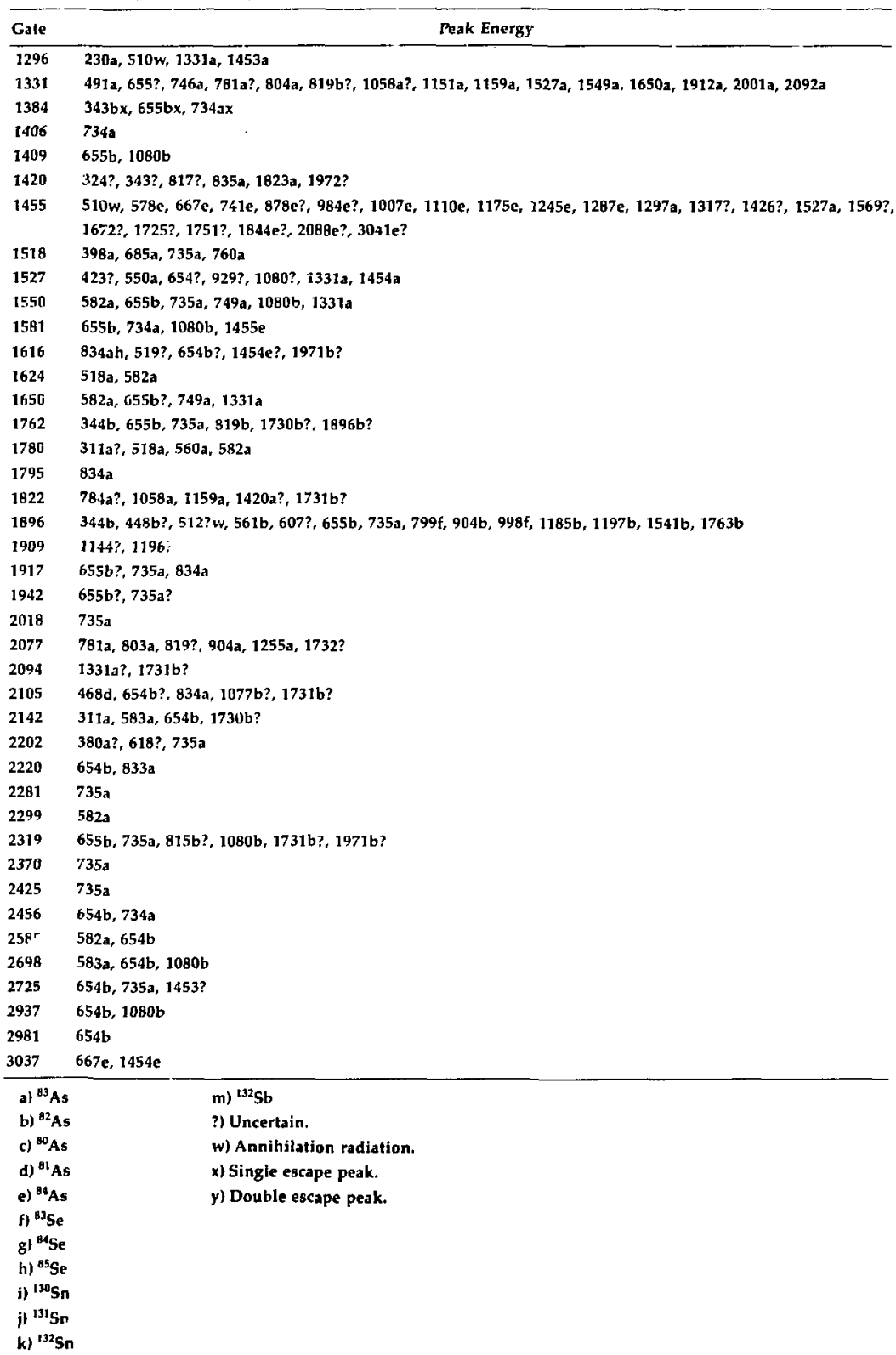


Table IV.2. In this table the basis for establishing each level is given. See the text for an explanation of the notation used in this table.

\begin{tabular}{|c|c|}
\hline Energy & Basis \\
\hline 0 & Ground state. (a) \\
\hline 228.9 & $\begin{array}{l}\text { I observe } \mathrm{C}(2077=803), \mathrm{C}(1331=745=803), \mathrm{C}(735=1113=803) \text {, and } \mathrm{C}(834=1013=803) \text {. Further the } 781 \text {, } \\
\text { keV gamma can be substituted for the } 803-\mathrm{keV} \text { gamma and the same observations hold. These transi- } \\
\text { tions are all intense, with the more intense transitions being placed lower in the decay scheme. I } \\
\text { determined that the } 803-\text { and } 781-\mathrm{keV} \text { transitions feed a level at } 2077 \mathrm{keV} \text { and the other transitions } \\
\text { cascade down from this level. These relationships established the levels at } 229,964,1063,1331,2077 \\
2858 \text {, and } 2881 \mathrm{keV} \text {. The } 229 \text { - and } 1331-k e V \text { levels have been observed in }{ }^{82} \mathrm{Se}\left(d_{,}, \mathrm{p}\right)^{83} \mathrm{Se} \text { (a) }\end{array}$ \\
\hline 540.3 & $\begin{array}{l}\text { I observe coincidences } \mathrm{C}(311=560=372), \mathrm{C}(311=932) \text { and } \mathrm{C}(582=518=372) \text {. These establish levels at } \\
540,582,1101 \text { and } 1473 \mathrm{keV} \text {. The } 582-\mathrm{keV} \text { transition is more intense than the } 518-k e V \text { transition } \\
\text { Similarly, the } 311-\mathrm{keV} \text { transition is stronger than the } 560-\mathrm{keV} \text { transition after the correction is made } \\
\text { for } 560-\mathrm{keV} \text { intensity in }{ }^{82} \text { As. This assignment was confirmed by the } 932-\mathrm{keV} \text { crossover to the } 560-\text { and } \\
372-\mathrm{keV} \text { pair. The } 540-, 582-\text { and } 1100-\mathrm{keV} \text { levels were seen in the (d,p) work. (a) }\end{array}$ \\
\hline 582.4 & See discussion for the 540-keV level. (a) \\
\hline 963.6 & See discussion for the 229 mkeV level. \\
\hline 1063.0 & See discussion for the 229-keV level. \\
\hline 1100.6 & See discussion for the 540-keV level. (a) \\
\hline 1265.3 & $\begin{array}{l}\text { This level is established from the } C(582=682=446) \text {. One half of } I(446) \text { is assigned to }{ }^{82} \text { As from the } 446- \\
\text { keV coincide:sce gate, making } I(682)>I(446) \text { in }{ }^{83} \text { As. Also I observe } C(1265=446) \text {, the ground state } \\
\text { transition. (a) }\end{array}$ \\
\hline 1296.2 & 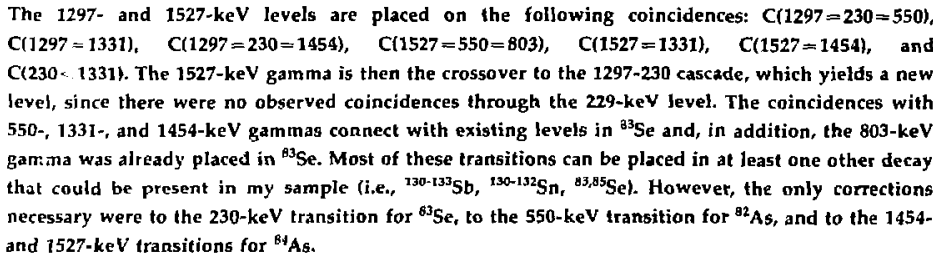 \\
\hline
\end{tabular}

1822.5 The $\mathrm{C}(1822=1057)$ and $\mathrm{C}(1331=491=1057)$ coincidences establish this level.

1907.9 This tevel is established from $\mathrm{C}(518<807), \mathrm{C}(560<807)$, and $\mathrm{C}(582<807)$.

1943.6 This level is established by $\mathrm{C}(734=980=914)$ and $C(1944=914)$ coincidences.

2077.0 See discussion for the 229-keV level.

2137.7 This level is placed from the $\mathrm{C}(834=1074)$ coincidence and the 1908-keV transition to the $229-\mathrm{keV}$ level. Also I nbserved $C(834<1144), C(834<1196), C(1077<1144), C(1077<1796), C(1908<1144)$ and C(1908 - 1196). The 1144- and 1196-keV transitions connect to separately established tevels.

2190.6 This level is placed on the basis of $\mathrm{C}(582=690), \mathrm{C}(582<1607), \mathrm{C}(690<1607)$, and $\mathrm{C}(690<2190)$.

2482.3 I based this level on $C(734=1518)$. (a)

$2545.9 \quad 1$ observed the $C\left(73_{4}=1581\right)$. Also, a level was observed at $2535-k e V$ in the $(\bar{d}, p)$ work. (a)

2679.2 The $\mathrm{C}(834=1615)$ places this level.

2724.6 This level is placed on the $(734=1760)$ and $C(582=518=1623)$.

2858.1 I observe the $C(734:=1895)$ coincidence, which established this level in ${ }^{83} \mathrm{Se}$. Al] other coincidences with the $3895-k e V$ gamma were assigned to ${ }^{82}$ As.

2880.7 This level is assigned on the strength of the $C(734=1917)$ coincidence. The only other coincidence with the 1917-keV gamma was the 834-keV transition, which is used in establishing the 2981-keV leval. 
Table IV.2. (Continued.)

\begin{tabular}{|c|c|}
\hline Energy & Basis \\
\hline 2971.I & This level is placed on $C(311-<2431)$ and $C(582<2389)$. \\
\hline 2981.1 & This level is placed on the basis of $C(1331=1649), C(734=2018)$ and $C(834=1918)$. \\
\hline 3167.5 & This level is placed on the $\mathrm{C}(734=2204)$ since the $2204-\mathrm{keV}$ gate has no other significant peaks. \\
\hline 3242.9 & This level is established on $C(734=2281)$ and $C(1822=1420)$ \\
\hline 3282.0 & This level is established from $C(734=2320)$ and $C(834=2220)$ coincidences. \\
\hline 3333.8 & This level is placed on $\mathrm{C}(734=2370)$. \\
\hline 3386.7 & This level is established from the $C(734=2424$ ! coincidence. \\
\hline 3424.0 & This level is established from the $C(734=2460)$ and $C(1331=2092)$ coincidences. \\
\hline 3463.8 & This level is placed on $C(582<2881)$. \\
\hline 3558.6 & This level is established from $\mathrm{C}(582<2975)$. \\
\hline 3690.2 & This level is estabtished from the $C(734=2729)$ coincidence. \\
\hline 3911.2 & This level is established from $\mathrm{C}(1331<2580)$. \\
\hline 4001.6 & This level is established from $\mathrm{C}(734=3038)$. \\
\hline
\end{tabular}

(a) Observed in the ${ }^{82} \mathrm{Se}(\overrightarrow{\mathrm{d}} . \mathrm{p})^{83} \mathrm{Se}$ experiment (MON78).

Table IV.3. Placement of gamma transitions in the decay of ${ }^{\mathrm{B}} \mathrm{As}$. The energy, uncertainty, relative intensity $(\mathrm{I}(734)=1000)$, uncertainty, initial level and final level are given. The notation $\mathrm{C}(. .$.$) is the same as before. Energies are in keV. Intensities are from the singles data$ unless otherwise specified. (For references to levels, see Table IV.2.)

\begin{tabular}{|c|c|c|c|c|c|}
\hline Energy & Error & Intensity & Error & Initial & Final \\
\hline \multirow[t]{2}{*}{42} & 2.0 & 0.005 & $100 \%$ & 582 & 540 \\
\hline & $C(31)$ & & & & \\
\hline \multirow[t]{2}{*}{135.8} & 3.0 & 0.2 & $100 \%$ & 1101 & 964 \\
\hline & $\mathrm{C} 1734$. & & & & \\
\hline \multirow[t]{2}{*}{157.2} & 1.3 & 0.9 & $100 \%$ & 1822 & 1665 \\
\hline & Crios2 & & & & \\
\hline \multirow[t]{2}{*}{165.3} & 1.2 & 0.1 & $120 \%$ & 1265 & 1101 \\
\hline & $C(518=$ & & & & \\
\hline \multirow[t]{2}{*}{192.0} & 2.0 & 0.7 & $120 \%$ & 1665 & 1473 \\
\hline & $\mathrm{C}(192=$ & (817) & & & \\
\hline \multirow[t]{2}{*}{195} & 2.0 & 1.0 & $80 \%$ & 1526 & 1331 \\
\hline & upper & & & & \\
\hline \multirow[t]{2}{*}{207} & 2.0 & 0.3 & $100 \%$ & 1473 & 1265 \\
\hline & upper & & & & \\
\hline \multirow[t]{2}{*}{230.0} & 0.2 & 10.0 & $10 \%$ & 1526 & 1296 \\
\hline & Cl1296 & see discuss & 1296 & & \\
\hline \multirow[t]{2}{*}{231} & 2.0 & 0.3 & $100 \%$ & 1331 & 1101 \\
\hline & upper 1 & & & & \\
\hline \multirow[t]{2}{*}{268.0} & 2.0 & 0.2 & $78 \%$ & $133 t$ & 1063 \\
\hline & іл 834 & les. & & & \\
\hline \multirow[t]{2}{*}{296} & 2.0 & 0.5 & $100 \%$ & 1822 & 1526 \\
\hline & upper & & & & \\
\hline \multirow[t]{2}{*}{311.5} & 0.1 & 30.7 & $3 \%$ & 540 & 229 \\
\hline & see dis & vel 540 & & & \\
\hline \multirow[t]{2}{*}{333.2} & 0.6 & 0.3 & $80 \%$ & 1665 & 1331 \\
\hline & singles & nit. & & & \\
\hline 350 & 2.0 & 0.3 & เบ้\% & 1822 & 1973 \\
\hline
\end{tabular}


Table IV.3. (Continued.)

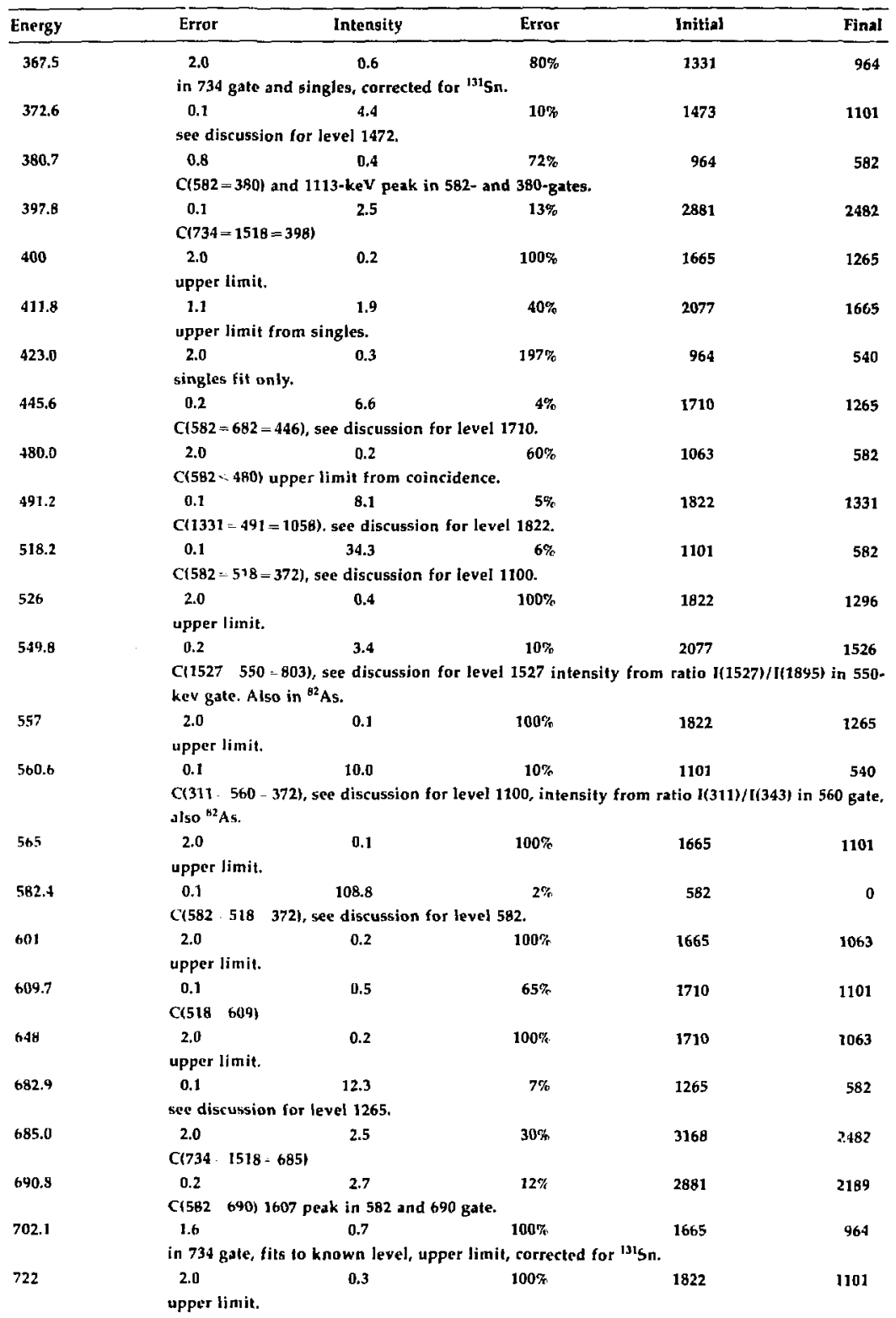


Table IV.3. (Continued.)

\begin{tabular}{|c|c|c|c|c|c|}
\hline Energy & Error & Intensity & Error & Initial & rinal \\
\hline \multirow[t]{2}{*}{734.9} & 0.1 & 1000.0 & $1 \%$ & 964 & 229 \\
\hline & sce dis & evel 963 & & & \\
\hline \multirow[t]{2}{*}{745.4} & 0.1 & 29.2 & $20 \%$ & 2077 & 1331 \\
\hline & $C(1331$ & & & & \\
\hline \multirow[t]{2}{*}{747} & 2.0 & 0.2 & $100 \%$ & 1710 & 964 \\
\hline & upper I & & & & \\
\hline \multirow[t]{2}{*}{748.8} & 0.2 & 12.3 & $20 \%$ & 1331 & 582 \\
\hline & $C(582$ & & & & \\
\hline \multirow[t]{2}{*}{759} & 2.0 & 0.3 & $100 \%$ & 1822 & 1063 \\
\hline & upper & & & & \\
\hline \multirow[t]{2}{*}{760.6} & 1.5 & 1.4 & $40 \%$ & 3243 & 2482 \\
\hline & $C(1518$ & & & & \\
\hline \multirow{2}{*}{781.1} & 0.1 & 32.3 & $7 \%$ & 2858 & 2077 \\
\hline & $\mathrm{C}(734=$ & $C(2077=78$ & & & \\
\hline \multirow[t]{2}{*}{791.0} & 1.0 & 0.4 & $80 \%$ & 1331 & 540 \\
\hline & in 311 & h 1331 leve & $t$ in 790 & & \\
\hline \multirow{2}{*}{803.8} & 0.1 & 92.8 & $2 \%$ & 2881 & 2077 \\
\hline & $\mathrm{C} 1734=$ & $(2077=80$ & & & \\
\hline \multirow[t]{2}{*}{806.0} & 1.0 & 3.1 & $50 \%$ & 2138 & 1331 \\
\hline & $C(1331$ & id 806 pea & vider in & an in 74 & \\
\hline \multirow[t]{2}{*}{807.5} & 0.3 & 2.5 & $30 \%$ & 1908 & 1101 \\
\hline & $C(518<$ & gles, fits, & & & \\
\hline \multirow[t]{2}{*}{812.0} & 2.0 & 0.3 & $125 \%$ & 2077 & 1265 \\
\hline & $\mathrm{C}(\mathrm{f} 8 \mathrm{B2}$ & $103<812)$ & & & \\
\hline \multirow[t]{2}{*}{817.2} & - & 2.7 & $52 \%$ & 2482 & 1665 \\
\hline & $\mathrm{C}(1083$ & le $818 \mathrm{from}$ & & & \\
\hline \multirow[t]{2}{*}{834.1} & 0.1 & 199.9 & $1 \%$ & 1063 & 229 \\
\hline & $\mathrm{C} 1834=$ & see discuss & 1063. & & \\
\hline \multirow[t]{2}{*}{845.0} & 1.5 & 1.0 & $50 \%$ & 1908 & 1063 \\
\hline & $\mathrm{Cl} 834<$ & gles. & & & \\
\hline \multirow[t]{2}{*}{871.0} & 1.5 & 1.5 & $80 \%$ & 1101 & 229 \\
\hline & $\mathrm{C}(1780$ & ted for 189 & pe. & & \\
\hline \multirow[t]{2}{*}{891} & 2.0 & 0.3 & & 1473 & 582 \\
\hline & upper I & & & & \\
\hline \multirow[t]{2}{*}{904.0} & 0.1 & 2.9 & $6 \%$ & 2981 & 2077 \\
\hline & $C(734=$ & & & & \\
\hline \multirow[t]{2}{*}{914.5} & 0.1 & 5.3 & $8 \%$ & 2858 & 1944 \\
\hline & $C(734=$ & & & & \\
\hline \multirow[t]{2}{*}{933.1} & 0.2 & 3.7 & $11 \%$ & 1473 & 540 \\
\hline & $C(31)=$ & & & & \\
\hline 944.0 & 0.3 & 1,8 & $100 \%$ & 1908 & 964 \\
\hline & $\mathrm{C}(734<$ & gles, but h & ong so $t$ & er limit. & \\
\hline 979.8 & 0.1 & 7.3 & $5 \%$ & 1944 & 964 \\
\hline & $\mathrm{C}(734=$ & e discussi & 944. & & \\
\hline 1010.0 & 2.0 & 1.0 & $100 \%$ & 2482 & 1473 \\
\hline & $\mathrm{C}(372<$ & & & & \\
\hline 1014.0 & 0.1 & 60.6 & $2 \%$ & 2077 & 1063 \\
\hline & $\mathrm{C}(834=$ & ee discuss & 2077. & & \\
\hline 1036.8 & 2.0 & 0.5 & $100 \%$ & 2138 & 1101 \\
\hline & $\mathrm{C}(51 \mathrm{~B}<$ & upper lin & & & \\
\hline 1036.8 & 2.0 & 1.0 & $60 \%$ & 2981 & 1944 \\
\hline & $C(979<$ & limit. & & & \\
\hline
\end{tabular}


Table IV.3. (Continued.)

\begin{tabular}{|c|c|c|c|c|c|}
\hline Energy & Error & Intensity & Error & Initial & Final \\
\hline \multirow[t]{2}{*}{1058.2} & 0.1 & 77.1 & $2 \%$ & 2881 & 1822 \\
\hline & $\mathrm{C}(1822$ & $31=491-1$ & ussion & & \\
\hline \multirow[t]{2}{*}{1074.0} & 0.7 & 15.0 & $15 \%$ & 2138 & 1063 \\
\hline & $C 1834=$ & iscussion fo & & & \\
\hline \multirow[t]{2}{*}{1082.9} & 0.5 & 7.1 & $20 \%$ & 1665 & 582 \\
\hline & $C(582=$ & iscussion fo & & & \\
\hline \multirow[t]{2}{*}{1113.4} & 0.1 & 360.9 & $3 \%$ & 2077 & 964 \\
\hline & $\mathrm{C}(734=$ & see discuss & 2077. & & \\
\hline \multirow[t]{2}{*}{1125.0} & 0.3 & 2.8 & $20 \%$ & 1665 & 540 \\
\hline & $\mathrm{C} 1311=$ & & & & \\
\hline \multirow[t]{2}{*}{1127.8} & 0.1 & 9.7 & $8 \%$ & 1710 & 582 \\
\hline & $C(582=$ & and $\mathrm{C}(582$ & 1169) & & \\
\hline \multirow[t]{2}{*}{1143.6} & $n .3$ & 2.3 & $25 \%$ & 3282 & 2138 \\
\hline & C(1908 & ected for ${ }^{13}$ & & & \\
\hline \multirow[t]{2}{*}{1151.1} & 0.4 & 6.0 & $8 \%$ & 2482 & 1331 \\
\hline & $C r 1331$ & & & & \\
\hline \multirow[t]{2}{*}{1158.7} & 0.1 & 13.1 & $15 \%$ & 2981 & 1822 \\
\hline & $\mathrm{Cr} 1822$ & $31=491=1$ & & & \\
\hline \multirow[t]{2}{*}{1169.3} & 0.1 & 14.3 & $6 \%$ & 2881 & 1710 \\
\hline & C(582 & & & & \\
\hline \multirow[t]{2}{*}{1196.0} & 0.6 & 1.4 & $22 \%$ & 3334 & 2138 \\
\hline & C(1907 & ected for & & & \\
\hline \multirow[t]{2}{*}{12183} & t.0 & 0.7 & $80 \%$ & $2482^{\circ}$ & 1265 \\
\hline & $\mathrm{C} 1682$ & ngles. & & & \\
\hline \multirow[t]{2}{*}{1240.0} & 0.5 & 0.8 & $50 \%$ & 1822 & 582 \\
\hline & $C(582=$ & with 1822 & mit. & & \\
\hline \multirow[t]{2}{*}{1243.0} & 1.0 & 1.0 & $80 \%$ & 1473 & 229 \\
\hline & $C(1 \geq 43$ & cidence lim & & & \\
\hline \multirow[t]{2}{*}{1257.0} & 0.2 & 2.3 & $18 \%$ & 3334 & 2077 \\
\hline & $\mathrm{C}(734$ & & & & \\
\hline \multirow[t]{2}{*}{1258.0} & 2.1 & 1.9 & $25 \%$ & 3168 & 1908 \\
\hline & $C(582$ & and 807 in & ntensity & dence. & \\
\hline \multirow[t]{2}{*}{1265.1} & 0.5 & 0.9 & $35 \%$ & 1265 & 0 \\
\hline & $C 1446$ & sity from si & & & \\
\hline \multirow[t]{2}{*}{1296.2} & 0.1 & 12.4 & $5 \%$ & 1296 & 0 \\
\hline & Ci1296 & see discus & Is 1527 a & & \\
\hline \multirow[t]{2}{*}{1326.8} & 1.0 & 1.5 & $50 \%$ & 1908 & 582 \\
\hline & $C(582=$ & limit from & & & \\
\hline \multirow[t]{2}{*}{1331.1} & 0.3 & 14.1 & $7 \%$ & 2858 & 1526 \\
\hline & $C(1527$ & $97=230=1$ & 1 goes $t$ & vel. & \\
\hline 1331.2 & 0.1 & 136.9 & $3 \%$ & 1331 & 0 \\
\hline & see dis & vel 1331. & & & \\
\hline 1367.0 & 1.1 & 0.1 & $100 \%$ & 1908 & 540 \\
\hline & upper & ble transiti & les. & & \\
\hline 1381.2 & 1.0 & 2.0 & $50 \%$ & 2482 & 1101 \\
\hline & C(562. & $518<1381$ ) & & & \\
\hline 1408.0 & 2.0 & 2.0 & $100 \%$ & 2881 & 1473 \\
\hline & $C 1372=$ & ngles. & & & \\
\hline 1439.5 & 0.2 & 2.3 & $15 \%$ & 2482 & 1063 \\
\hline & C1834 & & & & \\
\hline 1420.0 & 1.0 & 2.3 & $15 \%$ & 3243 & 1822 \\
\hline
\end{tabular}


Table IV.3. (Continued.)

\begin{tabular}{|c|c|c|c|c|c|}
\hline Energy & Error & Intensity & Error & Initial & Final \\
\hline 1454.7 & 0.3 & 52.5 & $6 \%$ & 2981 & 1526 \\
\hline \multicolumn{6}{|c|}{$C(1527,1455)$ and $C(1296=230=1454)$, sec discussion for level 1527 . } \\
\hline 1480.7 & 0.3 & 2.7 & $18 \%$ & 1710 & 229 \\
\hline \multicolumn{6}{|c|}{ from singles only; upper limit; also ${ }^{131} \mathrm{Sn}$. } \\
\hline \multirow[t]{2}{*}{1518.4} & 0.1 & 25.7 & $4 \%$ & 2482 & 964 \\
\hline & \multicolumn{5}{|c|}{$C(734=1518)$, see discussion for level 2482 . } \\
\hline \multirow[t]{2}{*}{1526.4} & 0.1 & 81.1 & $4 \%$ & 1526 & 0 \\
\hline & \multicolumn{5}{|c|}{$C(1527=1454)$, see discussion for level 1527} \\
\hline \multirow[t]{2}{*}{1537.2} & 1.6 & 2.2 & $37 \%$ & 2077 & 540 \\
\hline & \multicolumn{5}{|c|}{$\begin{array}{l}\mathrm{C}(311-1537) \text { is definite, but there is no } 803-\mathrm{keV} \text { coincidence. Placement is tentative, may } \\
\text { belong to }{ }^{42} \text { As. }\end{array}$} \\
\hline \multirow[t]{2}{*}{1548.8} & 0.1 & 30.7 & $4 \%$ & 2881 & 1331 \\
\hline & \multicolumn{5}{|c|}{$C(582=748 \ldots 1548)$ and $C(1331=1548)$} \\
\hline \multirow[t]{2}{*}{1582.3} & 0.2 & 3.0 & $17 \%$ & 2546 & 964 \\
\hline & \multicolumn{5}{|c|}{$C(734=1582)$} \\
\hline \multirow[t]{2}{*}{1596.6} & 0.8 & 0.9 & $42 \%$ & 2138 & 540 \\
\hline & \multicolumn{5}{|c|}{$C(311$ - 1597), singles, fits to this level. } \\
\hline \multirow[t]{2}{*}{1607.0} & 1.5 & 1.0 & $70 \%$ & 2191 & 582 \\
\hline & \multicolumn{5}{|c|}{$C(582=1607)$, see discussion for level 2189.} \\
\hline \multirow[t]{2}{*}{1615.5} & 0.1 & 22.7 & $3 \%$ & 2679 & 1063 \\
\hline & \multicolumn{5}{|c|}{$C(834-1615)$} \\
\hline \multirow[t]{2}{*}{1623.6} & 0.4 & 4.0 & $10 \%$ & 2725 & 1101 \\
\hline & \multicolumn{5}{|c|}{$C(582-518-1624)$} \\
\hline \multirow[t]{2}{*}{1641.0} & 0.4 & 0.2 & $100 \%$ & 2971 & 1331 \\
\hline & \multicolumn{5}{|c|}{ singles fit only. } \\
\hline 1649.2 & 0.1 & 22.0 & $3 \%$ & 2981 & 1331 \\
\hline & $C(1331$ & & & & \\
\hline 1664.6 & 0.3 & 1.8 & $20 \%$ & 1665 & $\mathbf{0}$ \\
\hline & singles & ence with & 6 of inte & & \\
\hline 1715.6 & 0.5 & 0.8 & $35 \%$ & 2981 & 1265 \\
\hline & C1582. & (1715) & & & \\
\hline 1761.4 & 0.1 & 7.3 & $5 \%$ & 2725 & 964 \\
\hline & $\mathrm{C} 1734=$ & ted for ${ }^{82} \mathrm{~A}$ & ssignme & gate. & \\
\hline 1780.2 & 0.1 & 16.2 & $4 \%$ & 2881 & 1101 \\
\hline & $\mathrm{C}(582=$ & & & & \\
\hline 1795.3 & 0.1 & 19.9 & $5 \%$ & 2858 & 1063 \\
\hline & $C(834$ & & & & \\
\hline 1818.0 & 0.2 & 6.1 & $10 \%$ & 2881 & 1063 \\
\hline & in 834 & les, intensi & cidence. & & \\
\hline 1822.5 & 0.1 & 81.7 & $2 \%$ & 1822 & 0 \\
\hline & $C(1822$ & iscussion fo & & & \\
\hline 1860.0 & 0.3 & 1.7 & $60 \%$ & 3334 & 1473 \\
\hline & $\mathrm{C}(372$ & & & & \\
\hline 1894.8 & 0.2 & 123.1 & $2 \%$ & 2858 & 964 \\
\hline & $\begin{array}{l}C(734=- \\
\text { Bote, en }\end{array}$ & $\begin{array}{l}{ }^{89} \text { As } C(6 \\
\text { d for diffe }\end{array}$ & $\begin{array}{l}3=560 \text { ), } \\
\text { n } 1895 \text { e }\end{array}$ & from It & n 1895 \\
\hline 1900.3 & 0.5 & 1.9 & $50 \%$ & 2482 & 582 \\
\hline & $\mathrm{C}(582$ & ngles. & & & \\
\hline 1908.9 & 0.1 & 19.9 & $6 \%$ & 2138 & 229 \\
\hline
\end{tabular}

$C(1908=1144), C(1908=1196)$, not placed from 1908 level because gammas feeding 1908 level are not in coincidence.

1912.0

0.5

$80 \%$

3243

1331

C(1331< 1912), intensity from coincidence. 
Table IV.3, (Continued.)

\begin{tabular}{|c|c|c|c|c|c|}
\hline Energy & Error & Intensity & Error & Initial & Final \\
\hline \multirow[t]{2}{*}{1917.3} & 0.1 & 129,1 & $3 \%$ & 2881 & 964 \\
\hline & \multicolumn{5}{|c|}{$C(734=1917)$ and $C(834=1919)$, split from 1917 gate. } \\
\hline \multirow[t]{2}{*}{1919.3} & 0.5 & 7.0 & $20 \%$ & 2981 & 1063 \\
\hline & \multicolumn{5}{|c|}{ see above 1917 gamma-ray. } \\
\hline \multirow[t]{2}{*}{1944,0} & 0.5 & 1.4 & $50 \%$ & 1944 & 0 \\
\hline & \multicolumn{5}{|c|}{ in 914 gate, see discussion for level 1944 . } \\
\hline \multirow[t]{2}{*}{2001.0} & 1.0 & 1.2 & $80 \%$ & 3334 & 1331 \\
\hline & \multicolumn{5}{|c|}{$C(734=2001)$} \\
\hline \multirow[t]{2}{*}{2017.9} & 0.1 & 12.7 & $8 \%$ & 2981 & 964 \\
\hline & \multicolumn{5}{|c|}{$C(734=2018)$} \\
\hline \multirow[t]{2}{*}{2077.0} & 0.1 & 249.5 & $2 \%$ & 2077 & 0 \\
\hline & \multicolumn{5}{|c|}{$C(2078=803)$, see discussion for level 2077} \\
\hline \multirow[t]{2}{*}{2092.0} & 1.0 & 1.4 & $20 \%$ & 3424 & $1,3 j 1$ \\
\hline & \multicolumn{5}{|c|}{$C(1331=2092)$} \\
\hline \multirow[t]{2}{*}{2098.3} & 1.2 & 0.9 & $80 \%$ & 2679 & 582 \\
\hline & \multicolumn{5}{|c|}{$C(582<2098)$} \\
\hline \multirow[t]{2}{*}{2104.2} & 0.2 & 3.7 & $10 \%$ & 3168 & 1063 \\
\hline & \multicolumn{5}{|c|}{$C(834=2105)$} \\
\hline \multirow[t]{2}{*}{2141.7} & 0.3 & 2.9 & $20 \%$ & 2679 & 540 \\
\hline & \multicolumn{5}{|c|}{$C(311=2141)$, no 518 , intensity assignments from coincidence. } \\
\hline 2142.5 & 0.7 & 0.7 & $50 \%$ & 2725 & 582 \\
\hline & $\mathrm{C}(582=$ & intensity & from co & & \\
\hline 2180.3 & 1.9 & 0.3 & $100 \%$ & 3243 & 1063 \\
\hline & $\mathrm{C}(834$. & gles. & & & \\
\hline 2190.6 & 0.7 & 1.1 & 73\% & 2191 & 0 \\
\hline & see disc & vel 2191. & & & \\
\hline 2204.6 & 0.1 & 213.4 & $1 \%$ & 3168 & 964 \\
\hline & $C(734=$ & cussion for & & & \\
\hline 2218.7 & 0.2 & 14.4 & $: 1 \%$ & 3282 & 1063 \\
\hline & $\mathrm{C} 1834=$ & & & & \\
\hline 2270.8 & D.5 & 1.5 & $29 \%$ & 2334 & 1063 \\
\hline & C1834 & ngles. & & & \\
\hline 2279.9 & 0.1 & 5.6 & $8 \%$ & 3243 & 964 \\
\hline & $C(734=$ & & & & \\
\hline 2299.2 & 0.1 & 8.9 & $6 \%$ & 2881 & 582 \\
\hline & C1582 = & & & & \\
\hline 2318.8 & 0.1 & 25.2 & $2 \%$ & 3282 & 964 \\
\hline & $C(734=$ & in ${ }^{82}$ As fro & ice. & & \\
\hline 2360.0 & 1.0 & 0.7 & $50 \%$ & 3424 & 1063 \\
\hline & $C(834=$ & gles. & & & \\
\hline 2370.4 & 0.1 & 11.4 & $5 \%$ & 3334 & 964 \\
\hline & $C(734:=$ & & & & \\
\hline 2388.3 & 0.9 & 2.3 & $20 \%$ & 2971 & 582 \\
\hline & $C(582$ & & & & \\
\hline 2423.1 & 0.3 & 5.8 & $12 \%$ & 3387 & 964 \\
\hline & $\mathrm{C}(734=$ & & & & \\
\hline 2429.5 & 0.5 & 2.9 & $18 \%$ & 2971 & 540 \\
\hline & $C(311=$ & gles. & & & \\
\hline 2449.9 & 0.2 & 9.7 & $8 \%$ & 2679 & 229 \\
\hline & fits fror & 30 coincider & s much & gles. & \\
\hline 2461.9 & 1.0 & 2.5 & $15 \%$ & 3424 & 964 \\
\hline
\end{tabular}


Table IV.3. (Continued.)

\begin{tabular}{|c|c|c|c|c|c|}
\hline Energy & Error & Intensity & Error & Initial & Final \\
\hline \multirow[t]{2}{*}{2580.0} & 2.0 & 2.0 & $100 \%$ & 3911 & 1331 \\
\hline & $C(1331$. & ingles. & & & \\
\hline \multirow[t]{2}{*}{2585.2} & 0.1 & 1.7 & $20 \%$ & 3168 & 582 \\
\hline & $C(532=$ & & & & \\
\hline \multirow[t]{2}{*}{2626.7} & 0.5 & 1.1 & $22 \%$ & 3690 & 1063 \\
\hline & $C(834=$ & ity split ba & idence. & & \\
\hline \multirow[t]{2}{*}{2629.0} & 1.5 & 0.3 & $70 \%$ & 3168 & 540 \\
\hline & $C(311)$ & & & & \\
\hline \multirow[t]{2}{*}{2699.6} & 0.1 & 5.2 & $5 \%$ & 3282 & 582 \\
\hline & $C(582=$ & & & & \\
\hline \multirow[t]{2}{*}{2724.6} & 0.1 & 1.8 & $35 \%$ & $272 ;$ & 0 \\
\hline & singles & ty corrected & $d^{82} A \leq f$ & nce. & \\
\hline \multirow[t]{2}{*}{2729.0} & 1.5 & 6.7 & $10 \%$ & 3690 & 964 \\
\hline & C(734 - & and intens & ncidence & & \\
\hline \multirow[t]{2}{*}{2742.5} & 0.2 & 4.4 & $10 \%$ & 3282 & 540 \\
\hline & $C(311)=$ & & & & \\
\hline \multirow[t]{2}{*}{ 2858,1 } & 0.1 & 170.0 & 27 & 2858 & 0 \\
\hline & bingles & corrected & s compc & & \\
\hline \multirow[t]{2}{*}{2865.0} & 1.2 & 0.7 & $100 \%$ & 3828 & 964 \\
\hline & $\mathrm{C}(734 \cdot$ & & & & \\
\hline \multirow[t]{2}{*}{2881.4} & 1.0 & 1.1 & $50 \%$ & 3464 & 582 \\
\hline & $C(582 \ldots$ & & & & \\
\hline \multirow[t]{2}{*}{2937.9} & 0.1 & 6.6 & $15 \%$ & 3168 & 229 \\
\hline & singles & & & & \\
\hline \multicolumn{6}{|l|}{2976.2} \\
\hline \multirow[t]{2}{*}{2981.2} & 0.5 & 15.4 & $4 \%$ & 2981 & 0 \\
\hline & singles & & & & \\
\hline \multirow[t]{2}{*}{3038.0} & 1.1 & 2.1 & $197 \%$ & 4001 & 964 \\
\hline & $C(735=-$ & & & & \\
\hline \multirow[t]{2}{*}{3242.8} & 0.1 & 35.1 & $2 \%$ & 3243 & 0 \\
\hline & singles & & & & \\
\hline \multirow[t]{2}{*}{3245.0} & 1.0 & 0.5 & $100 \%$ & 3828 & 582 \\
\hline & $C(5 B 2$ & & & & \\
\hline
\end{tabular}

Throughout this section, shorthand notation is used in an effor: to make the explanation as concise and clear as possible. The notation " $\mathrm{C}(\mathrm{a}<\mathrm{b})$ " means tha. there was a peak at energy $\mathrm{b}$ in coincidence spectrum $a$, but the reverse situation was not true. The notation " $C(a=b)$ " means that $C(a<b)$ and $C(b<a)$ were both true, the exclusion not holding. Expanding, $C(a=b=c)$ means that the above is true for all pairs. The notation " $C(a=b=c)$ and $C(a=b=d)^{\prime \prime}$ implies that $c$ and $d$ do not fit " $\mathrm{C}(\mathrm{c}=\mathrm{d})$ ". The notation I(a) means the intensity of the gamma-ray transition, a, which will be an energy in keV followed by any additional information necessary.

Levels have been established only on the basis of coincidence data and half-life information. Transitions which appeared only in the singles data were placed, based on energy and half-life, between previously established levels only. This procedure was followed to cope with the three major levels $\left({ }^{82} \mathrm{Se}\right.$, ground state, ${ }^{83} \mathrm{Se}$, ground state, and ${ }^{83} \mathrm{Se}^{\mathrm{m}}, 1 / 2^{-} 229-\mathrm{keV}$ state) which gave no coincidences. Examples are the 2858 - and the $1908-\mathrm{keV}$ transitions. The $2358-\mathrm{keV}$ transition was placed from the $2858-\mathrm{keV}$ level in ${ }^{83} \mathrm{Se}$ since this was the only place it would fit. On the basis of its energy, the 1908-keV transition depopulates either the 1908- or $2137-\mathrm{keV}$ level in ${ }^{\mathrm{B}} \mathrm{Se}$. Based on its weak, but definite, coincidences it was placed from the $2137-\mathrm{keV}$ level.

In most instances, energies and intensities were determined from the singles data. However, multiplets frequently required the use of the coincidence data to resolve these values. The 1895$\mathrm{keV}$ multiplet is a good example of the procedure used for multiplets. 
Using the coincidence data, the $1895-\mathrm{keV}$ transition was placed feeding the $654-\mathrm{keV}$ level in ${ }^{42} \mathrm{Se}$, in coincidence with the $654-\mathrm{keV}$ gamma ray, and feeding the $963-\mathrm{keV}$ level in ${ }^{83} \mathrm{Se}$, in coincidence with the 734-keV gamma ray. From data in the "Table of Isotopes" (TOI78), and my coincidence data, it was also placed in the decay of ${ }^{83} \mathrm{Se}^{8}$ feeding the $779-\mathrm{keV}$ level in ${ }^{43} \mathrm{Br}$, in coincidence with the $779-\mathrm{keV}$ gamma ray. The intensity of this portion of the $1895-\mathrm{keV}$ multiplet was obtained by normalizing the $718-\mathrm{keV}$ gamma ray from ${ }^{83} \mathrm{Se}^{\mathrm{g}}$ decay to the singles data. This gave $\mathrm{I}\left(1895,{ }^{\mathrm{K}} \mathrm{S} \mathrm{Se}^{\mathrm{b}}\right)=1.2 \%(4 \%)$ of $\mathrm{l}(1895$, total) when corrected for decay effects. (There is also a contrilution from ${ }^{131} \mathrm{Sn}$ decay of $0.001 \%$ of the multiplet intensity which was ignored.) The remaining intensity assigned to the two arsenic ciecays was $1(1895)=2.828 \mathrm{E} 6$. This intensity was split between the two arsenic decays based on $I(654)=7156(2 \%)$ and $I(734)=3442(3 \%)$ in the $1895-\mathrm{keV}$ coincidence gate, giving the intensity as

$\left(1895,{ }^{\mathrm{K}} \mathrm{As}\right)=\frac{1(734)}{1,734)+1 \mid 654 !}-1(1895)=9.19 \mathrm{E} 5(4 \% ;$

Thic energies were established from the energy difference of the $1895-\mathrm{keV}$ peak in the 654and $734-\mathrm{keV}$ gates, which was $0.9 \pm 0.2 \mathrm{keV}$. From the energies of the two components of the multiplet, the energy of the multiplet is

$I_{m 1}=\frac{l_{2} L_{2}+I_{3} L_{1}}{I_{2}+I_{1}}$

and

$I_{2}=I_{1}+0.9 \mathrm{keV}$

where subscripts $\mathrm{m}, 2$, and 3 refer to the multiplet, ${ }^{\mathrm{k}} \mathrm{As}$ and ${ }^{\mathrm{k} .3} \mathrm{As}$ respectively. The value for $E_{\mathrm{m}}$ is $1895.4+0.1 \mathrm{keV}$ and the intensities are known; solving for $E_{3}$ gives:

$l_{1}=l_{1+1} \quad \frac{I_{2}}{I_{2}+l_{1}}-0.9=1894.8 \pm 0.3$

Upper limits on law-intensity transitions were necessary for comparison to the model being invertigaters. These were evaluated primarily from the coincidence data, with support from the singlen dala when they were consistent. The usual mothod was to lnok for the specific photopeak in the appropriate gates and then establish an uncertainty. Freynently, however, the background nuad the uncertainty unreasonably large. This was true for the $137-\mathrm{keV}$ transition between the $1100-\mathrm{keV} 3 / 2+$ level and the $963-\mathrm{keV} 3 / 2$. The background at $137 \mathrm{keV}$ was large in the $734-\mathrm{keV}$ gate. An alternative procedure was to use the possible coincidence between the $734-\mathrm{kcV}$ gamma raly and the $1780-\mathrm{keV}$ gamma ray which feeds the $1100-\mathrm{keV}$ level. In the $1780-\mathrm{keV}$ gate the background at $734 \mathrm{koV}$ is approximately 2 counts/channel, which allowed the $137-\mathrm{keV}$ transition intensit: Io be sel at $0.6 \%$ of the $518-\mathrm{keV}$ transition intensity. The $157-\mathrm{keV}$ transition between the levels at 1822 and $1665 \mathrm{keV}$ was evaluated in much the same manner. Here, the 1082- and 582k(" "Iransitions appear in the $1057-\mathrm{keV}$ gate and the $582-\mathrm{keV}$ transition appears in the $1158-\mathrm{keV}$ gale, l'reviously, these transitions had been definitively placed, the 1082-keV transition depopulating the $1665 \cdot \mathrm{keV}$ level and the 1058 - and $1158 \mathrm{keV}$ transitions populating the $1822-\mathrm{keV}$ level. These coincidences showed the exislence of the $157-\mathrm{keV}$ transition connecting these (wo levels.

To llae the beta transition intensity in deducing the spin and parity of the levels in ${ }^{\text {A.3 }}$ Se, it was necessary to determine the intensity of the $M 4$ transition from the first $1 / 2$ level in ${ }^{\mathrm{B}} \mathrm{Se}$ and the bota intensity of this bevel.

Ihe transition inlensity from the $1 / 2 \quad 2,30-\mathrm{keV}$ level to the $9 / 2^{4}$ ground state can be estimaled from the systematic trend of the redeced transition probability of this $\mathrm{M} 4$ transition 
$(\mathrm{B}(\mathrm{M} 4))$ in ${ }^{89} \mathrm{Zr},{ }^{87} \mathrm{Sr}$, and ${ }^{85} \mathrm{Kr}$ where its intensity has been measured. The gamma-ray transition half-life is calculated from the the total half-life:

$\tau_{\gamma}=(1+\alpha) \times \tau_{\mathrm{T}} / 1_{1}$

where $\tau_{\gamma}$ and $\tau_{T}$ are the gamma-ray and total half-lives, $\alpha$ is the total $M 4$ internal conversion coefficient, and $\mathrm{I}_{\mathrm{l}}$ is the absolute transition intensity for gamma-ray plus internal conversion. The Weisskopf estimate of the half-life for an M4 transition (NDS80b) is

$\tau_{\mathrm{sp}}=\frac{4.84 E 4}{E^{9} A^{2}} \mathrm{~s}$.

The reduced transition probability in single particle units for this transition is

$\mathrm{B}(M 4)=\tau_{\mathrm{sp}} / \tau_{\gamma}$

In Table IV.4, J present the results of this procedure and use it to obtain an estimate of the M4 transition intensity in ${ }^{83} \mathrm{Se}$. J obtain an isomeric transition intensity in ${ }^{83} \mathrm{Se}$ of $0.008 \% \mathrm{~s}$ ) that I wil] be justified in ignoring this transition.

\section{Spin and Parity of ${ }^{83}$ As Ground State}

The intensity of the beta transition from ${ }^{83} \mathrm{As}$ to the tirst $1 / 2^{-}$level in ${ }^{83} \mathrm{Se}$ can provide evidence for the correct ground state spin and parity or ${ }^{83} \mathrm{As}$, but this intensity is unknown. However, it is known that ${ }^{73-81}$ As have ground state spin and parity $3 / 2^{-}$and those which decay to selenium have a strong branch to the first $1 / 2^{-}$level, as expected. The $\beta^{-}$intensities for decay to the first $1 / 2^{-}$state in selenium are given in Table JV.5.

To determine the heta transition intensity I performed a careful growth and decay experiment. Multichannel multiscaled gamina-ray data was accumulatec' from each of $55 \mathrm{samples}$ for 11 $\mathrm{min}$ in 20 time planes, as detailed in Table IV.6. This was enough time to follow the activity of the most intense gamma rays of both ${ }^{83} \mathrm{As}$ and ${ }^{83} \mathrm{Se}^{\mathrm{m}}$ to extinction.

In Fig. IV.1, I show schematically the decays from ${ }^{83} \mathrm{As}$ and ${ }^{83} \mathrm{Se}$ that must be followed in this measurement. Absolute gamma-ray and beta intensities (TOI78) were known for the decay of ${ }^{83} \mathrm{Se}^{\mathrm{m}}$. By taking the difference of the gamma-ray intensity populating and the beta intensity (as measured by the gamma-ray intensity) depopulating the ${ }^{83} \mathrm{Se}^{\mathrm{m}}$ level, I determined the beta intensity populating that level.

The photopeak amplitudes were extracted with the program FITEK, as described earlier. The amplitudes for the $391-\mathrm{keV}{ }^{113} \mathrm{Sn}$ (used for dead time corrections), 734-keV ${ }^{83} \mathrm{As}$, anu $674-$, 988-, $1030-$, and $2051-\mathrm{keV}{ }^{83} \mathrm{Se}^{\mathrm{m}}$ photopeaks are shown in Table IV.6 for all of the time planes. The long-lived components of the 988- and 1030-keV photopeaks were determined to be from ${ }^{132} \mathrm{Sb}$ 'the daughter of ${ }^{132} \mathrm{Sn}$ ) and ${ }^{89} \mathrm{Rb}$ decay, respectively. These activities were identified and corrections made based on photopeak intensities, energies, and half-lives in t.ie nineteenth and twentieth plines. Additionally, the antimony was anticipated from the presence of ${ }^{132} \mathrm{Sn}$ in all of my other arsenic spectra.

The intensity of the gamma rays used was compared to calculated decay curves to verify that the gamma rays had the correct half-life. This was done by dividing the observed number of counts by the calculated ction that should have decayed in the given plane. The results of this analysis for the 674- and 988-keV gamma rays are shown in Figs. IV.2a and IV.2b. The values are computed assuming half-lives of 65,70 , and $75 \mathrm{~s}$. (The correct half-life has been measured as $70.4 \mathrm{~s}$ (MEY81).) The effect of a long-lived contaminant is evident in the 988-keV gamma ray. In the present work, the $734-\mathrm{keV}$ gamma ray shows a $12.4-\mathrm{s}$ half-life and this value was used in these calculations. 
Table IV.4. Values for the half-life, gamma-ray energy $\left(E_{\gamma}\right)$, gamma-ray intensity $\left(I_{\gamma}\right)$, and internal conversion coefficient (ICC) used in evaluating the systematic behavior of the $B(M 4)$ in some $N=49$ nuclei. The trend in the $B(M 4)$ was established using the first three nuclei and the LiM4) for ${ }^{83}$ Se was estimated and the gamma half-life and gamma branching ratio calculated. The values I calculated are given in parentheses.

\begin{tabular}{|c|c|c|c|c|}
\hline & \multicolumn{4}{|c|}{ Nuclide } \\
\hline & ${ }^{89} \mathrm{Zr}$ & ${ }^{87} \mathrm{Sr}$ & ${ }^{85} \overline{K r}$ & ${ }^{83} \mathrm{Se}$ \\
\hline Half-life & $4.18 \mathrm{~min}$ & $2.80 \mathrm{~h}$ & $4.48 \mathrm{~h}$ & $70 \mathrm{~s}$ \\
\hline $\mathrm{E}_{\sim}(\mathrm{keV})^{2}$ & 588 & 389 & 305 & 229 \\
\hline$I_{j}(\%)^{a, b}$ & 93.8 & 99.7 & 21 & $(0.01)$ \\
\hline $\mathrm{ICC}^{\mathrm{c}}$ & 2.8B & 2.36 & 1.93 & 1.56 \\
\hline Gamma half-life (s) & $1.04 \mathrm{E} 3$ & $3.40 \mathrm{E} 4$ & 2.25E5 & (2.22E6) \\
\hline$T_{n p}(s)^{d}$ & $3.17 \mathrm{E} 3$ & $1.37 \mathrm{E5}$ & $1.28 E 6$ & 1.77E7 \\
\hline B(M4) (SPU) & 3.06 & 4.03 & 5.69 & $(8.04\}$ \\
\hline
\end{tabular}

TO178.

' Intensity of the isomeric transition, gamma plus internal conversion.

c ROS78.

'Weisskopf estimate.

Table IV.5. Values for selected beta branching intensities, $E_{\beta r} Q_{\beta}$ and $\log \left(f_{t} t\right)$ values for odd-mass arsenic. The beta transition of interest is from the arsenic ground state to the first $1 / 2 *$ state in the daughter selenium.

\begin{tabular}{|c|c|c|c|c|c|}
\hline Isotope & $\begin{array}{c}\mathrm{E}\left(1 / 2^{-}\right) \\
\mathrm{MeV}\end{array}$ & $\%-\beta$ & $\begin{array}{c}\text { Hall- } \\
\text { life }\end{array}$ & $\underset{\mathbf{M e V}}{\mathbf{Q}_{\boldsymbol{g}}}$ & $\log \left(f_{0} t\right)$ \\
\hline${ }^{77} \mathrm{As}$ & 0.0 & 97.5 & $38.8 \mathrm{~h}$ & 0.69 & 5.7 \\
\hline${ }^{79} \mathrm{As}$ & 0.096 & 95.0 & $9.0 \mathrm{~min}$ & 2.2 & 5.2 \\
\hline${ }^{81} \mathrm{As}$ & 0.0 & 67,0 & $33.0 \mathrm{~s}$ & 3.75 & 5.2 \\
\hline${ }^{83}$ As & 0.23 & 0.3 & $13.0 \mathrm{~s}$ & 5.46 & 7.9 \\
\hline
\end{tabular}

Tabie IV.6. This table contains the raw data from the multichannel multiscaled data for the arsenic ground-state beta decay. The values given are the photopeak amplitudes and percent uncertainties from the program FITEK. The time values are the length of each plane and cumulative time to the end of the plane. Under each isotope heading are the energies of each photopeak for that isotope and the associated detector relative efficiency.

\begin{tabular}{|c|c|c|c|c|c|c|c|}
\hline \multirow[t]{2}{*}{$\begin{array}{l}\text { Plane } \\
\text { number }\end{array}$} & \multirow[t]{2}{*}{$\begin{array}{c}\text { Time } \\
5\end{array}$} & \multirow{2}{*}{$\frac{{ }^{113} \mathrm{Sn}}{392}$} & \multirow{2}{*}{$\frac{{ }^{83} \mathrm{As}}{734}$} & \multicolumn{4}{|c|}{${ }^{83} S e^{m}$} \\
\hline & & & & 674 & 988 & 1030 & 2051 \\
\hline & & - & 2.2 & 2.4 & 1.7 & 1.7 & 0.9 \\
\hline \multirow[t]{2}{*}{1} & 0.2 & 416.4 & - & - & - & - & - \\
\hline & 0.2 & 19.0 & & & & & \\
\hline \multirow[t]{2}{*}{2} & 0.4 & 737.7 & 666.0 & - & - & $-\cdot$ & - \\
\hline & 0.6 & 11.0 & 15.6 & & & & \\
\hline \multirow[t]{2}{*}{3} & 0.5 & 1212.7 & 1020.0 & - & - & - & - \\
\hline & 1.1 & 8.0 & 9.0 & & & & \\
\hline \multirow[t]{2}{*}{4} & 0.5 & $1 \mathrm{v67.8}$ & 1018.0 & - & - & - & - \\
\hline & 1.6 & 8.0 & 8.0 & & & & \\
\hline
\end{tabular}


Table IV.6. (Continued.)

\begin{tabular}{|c|c|c|c|c|c|c|c|}
\hline \multirow{3}{*}{$\begin{array}{c}\begin{array}{c}\text { Plane } \\
\text { number }\end{array} \\
5\end{array}$} & \multirow{2}{*}{$\begin{array}{c}\begin{array}{c}\text { Time } \\
\mathrm{s}\end{array} \\
1.0\end{array}$} & \multirow{2}{*}{$\frac{{ }^{113} \mathrm{Sn}}{2110.5}$} & \multirow{2}{*}{$\frac{{ }^{63} \mathrm{As}}{2061.0}$} & \multicolumn{4}{|c|}{${ }^{83} \mathrm{Se}^{m}$} \\
\hline & & & & - & - & - & - \\
\hline & 2.6 & 6.0 & 7.0 & & & & \\
\hline \multirow[t]{2}{*}{6} & 1.0 & 2444.0 & 2091.0 & - & - & - & - \\
\hline & 3.6 & 6.0 & 7.0 & & & & \\
\hline \multirow[t]{2}{*}{7} & 2.0 & 4830.0 & 3858.0 & - & - & - & - \\
\hline & 5.6 & 5.0 & 4.0 & & & & \\
\hline \multirow[t]{2}{*}{8} & 2.0 & 4612.0 & 3630.0 & - & - & - & - \\
\hline & 7.6 & 6.0 & 4.0 & & & & \\
\hline \multirow[t]{2}{*}{9} & 5.0 & 12342.0 & 7430.0 & 110.0 & 273.0 & - & 45.0 \\
\hline & 12.6 & 7.0 & 3.0 & $8 \div .0$ & 23.0 & & 78.0 \\
\hline \multirow[t]{2}{*}{10} & 5.0 & 12939.0 & 5605.0 & 217.0 & 356.0 & 276.1 & 74.0 \\
\hline & 17.6 & 3.0 & 4.0 & 24.0 & 22.0 & 15.0 & 22.0 \\
\hline \multirow[t]{2}{*}{11} & 10.0 & 25452.0 & 7263.0 & 495.0 & 496.0 & 532.4 & 96.0 \\
\hline & 27.6 & 2.0 & 2.6 & 18.0 & 10.0 & 10.0 & 25.0 \\
\hline \multirow[t]{2}{*}{12} & 10.0 & 26064.0 & 4338.0 & 549.0 & 493.0 & 581.1 & 154.0 \\
\hline & 37.6 & 5.0 & 2.6 & 14.0 & 10.0 & 16.0 & 13.0 \\
\hline \multirow[t]{2}{*}{13} & 15.0 & 39449.0 & 3176.0 & 795.0 & 748.0 & 953.2 & 237.0 \\
\hline & 52.6 & 1.5 & 5.0 & 9.0 & 8.0 & 7.1 & 10.0 \\
\hline \multirow[t]{2}{*}{14} & 15.0 & 39119.0 & 1369.0 & 798.0 & 616.0 & 861.9 & 184.0 \\
\hline & 67.6 & 0.9 & 9.0 & 8.0 & 8.0 & 12.4 & 16.0 \\
\hline \multirow[t]{2}{*}{15} & 15.0 & 39552.0 & 639.0 & 692.0 & 468.0 & 718.0 & 185.0 \\
\hline & 82.6 & $1 . \mathrm{t}$ & 16.0 & 7.0 & 10.0 & 6.8 & 16.0 \\
\hline \multirow[t]{2}{*}{16} & 30.0 & 77429.0 & 208.0 & 1099.0 & 897.0 & 1340.2 & 300.0 \\
\hline & 112.6 & 0.7 & 45.0 & 10.0 & 7.0 & 15.1 & 11.0 \\
\hline \multirow[t]{2}{*}{17} & 60.0 & 154076.0 & 298.0 & 1549.0 & 1259.0 & 1781.5 & 406.0 \\
\hline & 172.6 & 1.1 & 20.0 & 7.0 & 6.0 & 13.2 & 10.0 \\
\hline \multirow[t]{2}{*}{18} & 120.0 & 316737.0 & 142.0 & 1192.0 & 1146.0 & 2053.8 & 315.0 \\
\hline & 292.6 & 1.7 & 29.0 & 7.0 & 6.0 & 4.6 & 13.0 \\
\hline \multirow[t]{2}{*}{19} & 180.0 & 4642911.0 & - & 417.0 & 482.0 & 1616.3 & 126.0 \\
\hline & 472.6 & 1.5 & - & 15.0 & 10.0 & 5.6 & 20.0 \\
\hline \multirow[t]{2}{*}{20} & 180.0 & 4650711.0 & - & 0.0 & 125.0 & 1202.7 & 0.0 \\
\hline & 652.6 & 0.6 & & 0.0 & j8.J & 5.3 & 0.0 \\
\hline
\end{tabular}




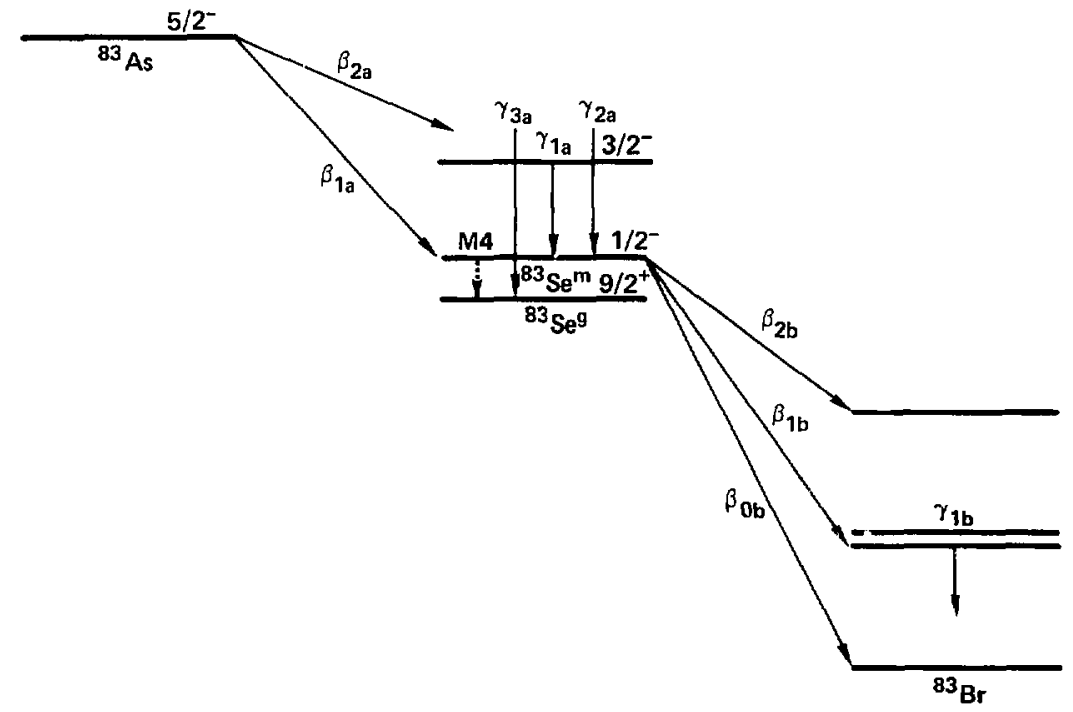

Fig. IV.1. A partial decay scheme showing the gamma transitions, $\gamma_{1 a}$ and $\gamma_{1 b}$, followed in the growth and decay experiment. The relative beta transition intensity for $\beta_{1 \mathrm{a}}$ was determined. Relative gamma transition intensities were knowrt for the decay of ${ }^{83} \mathrm{Se}^{m}$ (MEY81) and were measured for the decay of ${ }^{83} \mathrm{As}$ (this work). Relative beta transition intensities for the decay of ${ }^{83} \mathrm{Se}^{\mathrm{m}}$ were known (SCH68). The labels refer to: $\beta_{2 a}$, beta transitions from the decay of ${ }^{83}$ As going to the levels above the ${ }^{83} \mathrm{Se}^{\mathrm{m}}$ state; $\gamma_{1 \mathrm{a}}$, the $734-\mathrm{keV}$ transition in ${ }^{83} \mathrm{Se} ; \gamma_{1 b}$, gamma transitions fed by the decay of only ${ }^{83} \mathrm{Se}^{\mathrm{m}}$; and $\beta_{0 \mathrm{~b}}, \beta_{1 \mathrm{~b}}, \beta_{2 \mathrm{~b}}$, the beta transitions from ${ }^{83} \mathrm{Se}^{\mathrm{m}}$ which feed the ievels in ${ }^{83} \mathrm{Br}$ at 0,1 , and $2 \mathrm{MeV}$, respectively. The gamma rays are: $\gamma_{1 a^{\prime}}$ the 734-keV garsma ray $w$ hich was measured in this experiment; $\gamma_{2,}$, collectively, the other gamma rays feeding the $1 / 2_{1}^{-}$in ${ }^{83} \mathrm{Se} ; \gamma_{3 a}$ the gamma rays feeding the $9 / 2_{1}^{+}$in ${ }^{83} \mathrm{Sc}$; and $\gamma_{1 \mathrm{~b},}$ a typical gamma ray measured in the decay of ${ }^{83} \mathrm{Se}^{\mathrm{m}}$. The label $M 44$ refers to the possible $M 4$ transition :vhich was estimated to have zero intensity. 


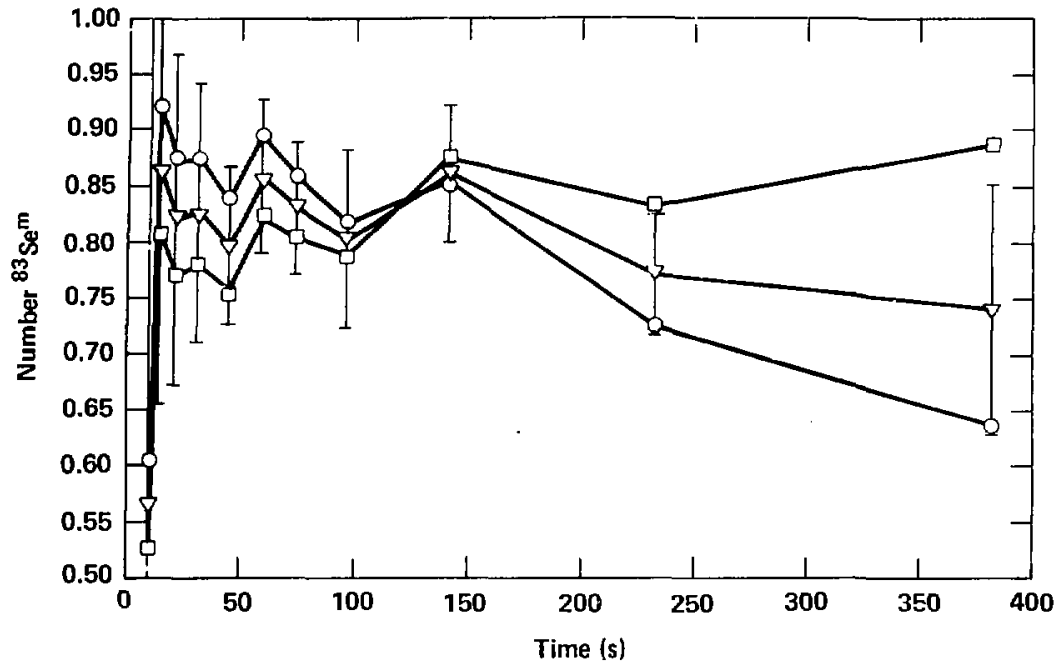

Fig. IV.2a. Number of ${ }^{83} \mathrm{Se}^{\mathrm{m}}$ nuclei at zero time $\left(\mathrm{N}_{\mathrm{o}}\right)$ calculated for each time plane in the growth and decay experiment plotted against time for the $674-\mathrm{keV}$ gamma transition. Calculations are based on 65- ( $\square), 70-(\nabla)$, and 75-5 $(O)$ half-lives. The 70-s half-life gives the most constant $N_{0}$. Error bars are indicated for the 70 -s half-life.

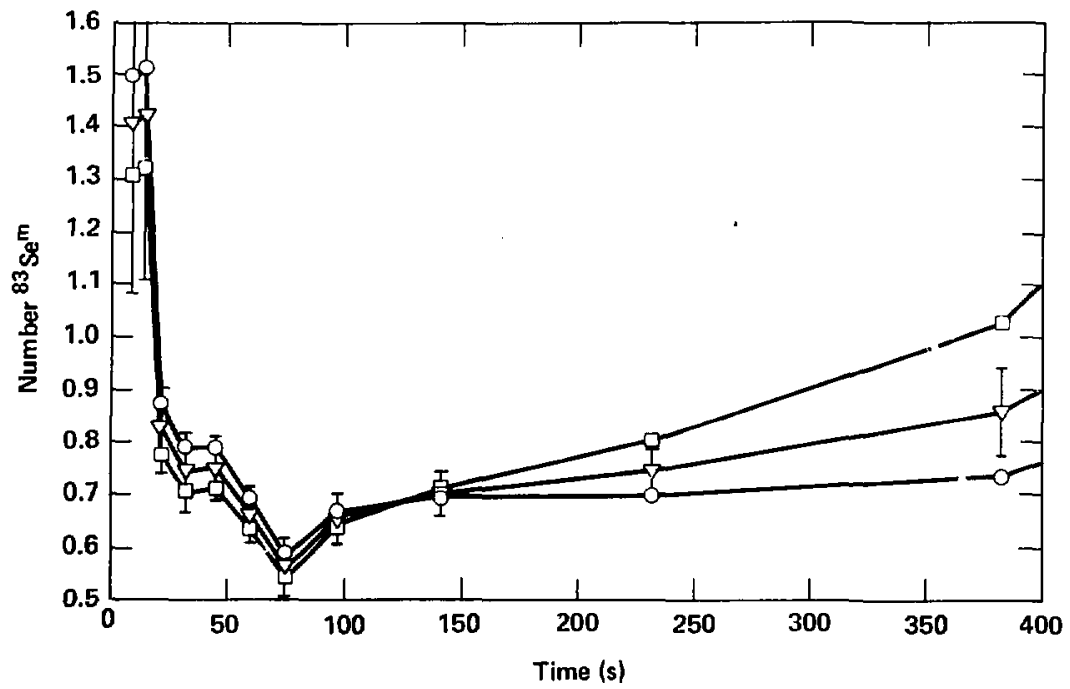

Fig. IV.2b. The plot is the same as Fig. IV.2a, but the values displayed are for the 988-keV gamma ray. Here the effect of the ${ }^{132} \mathrm{Sb}$ contaminant is readily apparent. 
The individual spectra then were summed over the ninth through the eighteenth planes (7.6 to $292.6 \mathrm{~s}$ ) and the last two planes (292.6 to $652.6 \mathrm{~s}$ ) to give two final spectra and photopeak intensities extracted as before. This procedure eliminated the uncertainty in the dead time in the first eight planes while retaining most of the peak intensity in the first sum $(60 \%$ of the arsenic and $90 \%$ of the selenium data were retained). The second sum was then used to correct for the long-lived contaminants using coefficients calculated from the decay curve.

The photopeak areas were next corrected for the fraction of decays observed over the time interval and relative detector efficiency. The decay fractions were calculated from the Bateman equations (FRI65) and the dead times calculated from the ${ }^{113} \mathrm{Sn}$ data in Table IV.6. The results of these calculations are shown in Table IV.7.

From the data for each gamma ray from the decay of ${ }^{83} \mathrm{Se}^{\mathrm{m}}$, I obtain the total number of decays of ${ }^{83} \mathrm{Se}^{\mathrm{m}}, \mathrm{N}_{0}$, which must be equal to the number of decays populating this level

$N_{0}=C\left(\gamma_{10}\right) / 1\left(\gamma_{16}\right)=C\left(\gamma_{10}\right) / I\left(\gamma_{10}\right)$

where $C$ is the corrected counts, $I$ is the absolute intensity considering decay to ${ }^{83} \mathrm{Se}^{\mathrm{m}}$ only, and $\gamma_{1 \mathrm{a}}$ and $\gamma_{1 \mathrm{~b}}$ refer to gamma rays following the decay of ${ }^{83} \mathrm{As}$ and ${ }^{83} \mathrm{Se}^{\mathrm{m}}$, respectively. The absolute gamma intensity is related to the relative intensities by

$l\left(\gamma_{10}\right)=\frac{I^{\prime}\left(\gamma_{1, a}\right)}{I^{\prime}\left(\gamma_{1,}\right)+I^{\prime}\left(\gamma_{2 a}\right)+I^{\prime}\left(\beta_{10}\right)}$

where the I' are relative inten.ities, $\gamma_{10}$ is the $734-\mathrm{keV}$ gamma-ray transition feeding the $1 / 2^{-}$ level, $\gamma_{2,}$ labels all other gamma-ray transitions feeding the $1 / 2^{-}$level as measured in this experiment and $\beta_{1,}$ is the $\beta$ transition to the $1 / 2^{-}$level. Solving Eqs. (IV.8) and (IV.9) for the $\beta^{-}$ intensity yields

Table iV.7. Data development for evaluating the intensity of the $\beta^{--}$branch to the ${ }^{83} \mathrm{Se}^{\mathrm{m}}$ state. Counts 1 and 2 are from the two summed planes $(7.6$ to $292.6 \mathrm{~s}$ and 292.6 to $652.6 \mathrm{~s}$, respectively)asindicatedinthetext. $N_{0}$ (raw)isfromdecayonly, notincludingthedetectorefficiencyor the absolute gamma intensity.

\begin{tabular}{|c|c|c|c|c|c|c|}
\hline $\begin{array}{l}\text { Energy } \\
\text { keV }\end{array}$ & $\begin{array}{c}\text { Counts } \\
1\end{array}$ & $\begin{array}{c}\text { Counts } \\
2\end{array}$ & $N_{0}\left(\operatorname{raw}^{\mu}\right)$ & $\begin{array}{l}\text { detector } \\
\text { efficiency }\end{array}$ & $\begin{array}{c}\text { Gamma } \\
\text { Intensity }\end{array}$ & $N_{0}$ (true) \\
\hline \multirow[t]{2}{*}{674} & 7371 & 432 & 8035 & 2.403 & $0.137^{c}$ & 24406 \\
\hline & (3.1) & $(14.3)$ & $(3.1)$ & & $(7.2)$ & $(7.8)$ \\
\hline \multirow[t]{2}{*}{734} & $29820^{d}$ & 0 & 46304 & 2.232 & $1.000^{\circ}$ & 20745 \\
\hline & $(3,8)$ & - & (3.8) & & $(2.6)$ & $(4.6)$ \\
\hline \multirow[t]{2}{*}{988} & 6297 & 746 & $6114^{t}$ & $1.7 q 1$ & $0.138^{\circ}$ & 25448 \\
\hline & $(3.1)$ & $(10.2)$ & (5.2) & & $(7.2)$ & (8.9) \\
\hline \multirow[t]{2}{*}{1030} & 9427 & $289 \mathrm{C}$ & $7: 166^{8}$ & 1.677 & $0.194^{c}$ & 23255 \\
\hline & (3.3) & $(5.2)$ & (5.2) & & $(10.2)$ & (9.2) \\
\hline \multirow[t]{2}{*}{2051} & 2014 & 130 & 2195 & 0.969 & $0.089^{5}$ & 25451 \\
\hline & $(5.2)$ & $(20.5)$ & (5.2) & & $(7.6)$ & (8.6) \\
\hline
\end{tabular}

a Counts corrected for decay and contamination effects only.

"LIN80.

'Absolute intensity: intensities relative to $356-\mathrm{keV}$ transition from MEY81. Absoluke iormalization of this transition is from SCH68. MEYB1, and this work.

$\mathrm{d}$ Sum for this transition is from $7.6 \mathrm{~s}$ to $82.6 \mathrm{~s}$.

- This is a relative intensity; total gamma intensily to this level is $\mathbf{1 . 2 7 2}$.

${ }^{1}$ Corrected for ${ }^{132} \mathrm{k} 5 \mathrm{~b}$ daughter of ${ }^{132} \mathrm{Sn}$.

k Corrected for direct "Rb. 


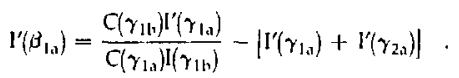

The key piece of information needed to obtain the absolute intensities in the decay of ${ }^{43} \mathrm{Se}^{\mathrm{m}}$ was the intensity of the beta branch to the ${ }^{{ }^{*} 3} \mathrm{Br}$ ground state, $L\left(\beta_{0 \mathrm{~b}}\right)$. Using the accepted value for $\left[\left(\beta_{01}\right)\right.$ of $31 \%$ (e.g., TOI78) resulted in ${ }^{83} \mathrm{Se}^{\mathrm{m}}$ having significantly more transitions entering than leaving, an impossible result. Schussler $(5 \mathrm{CH} 68)$ measured relative beta intensities with a magnetic spectrometrr and from these relative intensities deduced the absolute intensities. However, he failed to account for the significant contribution of ${ }^{\mathrm{H}}$ Se decay to his beta spectrum. In Appendix 2 this problem is analyzed in detail.

To normalize the beta and gamma-ray intensities 1 assumed instead that the $2880-\mathrm{keV}$ beta group intensity supplied exactly the beta intensity needed by the levels near $1 \mathrm{MeV}$ as determined from gamma-ray spectroscopy (see App. 2). With this normalization, 1 determined absolute intensities for the ground state beta branch from ${ }^{83} \mathrm{Se}^{\mathrm{m}}(38.4 \pm 2.7) \%$, and for the $356-\mathrm{keV}$ gamma-ray transition $(14.9 \pm 0.9) \%$.

Using these values and the data given in Tables IV.6 and IV.7, I found the absolute beta intensity to ${ }^{43} \mathrm{Se}^{\mathrm{m}}$ to be $(-4 \pm 3) \%$. From this I determined that the beta transition intensity from ${ }^{43}$ As to the first excited $1 / 2$ state in ${ }^{83} \mathrm{Se}$ is zero. From this zero beta intensity, I conclude that the ${ }^{8.3}$ As ground state has a spin and parity of $5 / 2^{*}$.

Fulther confirmation of the $5 / 2$ spin and parity assignment is ohtained from the transitions involving the $2077 \cdot \mathrm{k}\left(\mathrm{PV}\right.$ level in ${ }^{8.3} \mathrm{Se}$. This level has an allowed $\log (\mathrm{ft})=5.0$ and a strong gammaray transition to the $9 / 2$ ground state. The only possible spin and parity issignments are $7 / 2$ for the 2077 -keV level and $5 / 2$ for the ${ }^{83}$ As ground state.

However, the negative intensity for the $\beta$ transition to the ${ }^{83} \mathrm{Se}^{\mathrm{m}}$ iscmeric state is physically impossible and does not allow the calculation of a $\log (\mathrm{ft})$ value. Using Bayes' pinciple it is possible to obtain the most probable $\beta^{-}$intensity for the observed data. Assuming that the intensity must be greater than zero, a probability distribution function with that property is generated and an expected value calculated from the inputs. Equation (IV.10) was rearranged to give

$I^{\prime}\left(\beta_{1 \mathrm{~s}}\right)=-\frac{\alpha C_{i n}-\beta C_{a}}{C_{u}}$

where $C$ is the number of counts corrected for contamination but nothing else, $a$ and $b$ label counts from the decay of ${ }^{83} \mathrm{As}$ and ${ }^{43} \mathrm{Se}$, respectively, and $\alpha$ and $\beta$ are constants which include decay effects, intensities, and detector efficiencies. Using the algorithm developed by Vincent (VIN82) and Eq. (IV.11), I calculated that the $\beta^{-}$intensity to ${ }^{83} \mathrm{Se}^{\mathrm{m}}$ was $(0.3 \pm 0.2) \%$ absolute and $\log (\mathrm{ft})=7.9$. This $\log (\mathrm{ft})$ value agrees with similar decays from ${ }^{81} \mathrm{Se},{ }^{69} \mathrm{As}$, and ${ }^{65} \mathrm{Ni}$, which have $\log (\mathrm{ft}$ ) values ranging from 7.4 to greater than 9.8 (TOl78).

The last intensity needed is the $\beta^{-}$branch to ${ }^{83} \mathrm{Se}^{\mathrm{s}}$. This was estimated from the $\log \left(f_{1} t\right)$ for $\beta$ decay of ${ }^{7 /} \mathrm{Zn}$ and ${ }^{85} \mathrm{Kr}$ to $5 / 2$ states. All of these $\beta^{-}$decays transform a $1 \mathrm{~g} 9 / 2$ neutron to a $115 / 2$ proton so 1 expect the $\beta$ decay to have a $\log (f, t)$ similar to those in ${ }^{71} \mathrm{Zn}$ and ${ }^{85} \mathrm{Kr}$, which have values of 9.5 and 9.4 respectively. Using this value in the ${ }^{83}$ As decay gives a $\beta^{-}$intensity of $0.7 \%$, which does not alter my prior intensity calculations.

This result for ${ }^{43}$ As $\beta^{-}$decay is markedly different from other odd-mass arsenic $\beta^{-}$decays to the first $1 / 2$ state in selenium. In Table IV.5, I give the beta transition intensity for beta decay from the arsenic ground state to the first $1 / 2$ state in selenium. For ${ }^{77,79.81} \mathrm{As}$, the ground state is $3 / 2$ and the transition has high intensity, but for ${ }^{83}$ As I have found this intensity to be $0.3 \%$. In Fig. IV.3, I give the systematics for the odd-mass arsenics (HOF81). In odd-mass ${ }^{75-81}$ is, the first $5 / 2^{-}$is near $300 \mathrm{keV}$. It could readily become the ground state. The $5 / 2^{-}$spin and parity assignment is consist"nt with the lack of beta intensity to the first $3 / 2^{-}$state in ${ }^{83}$ Se. While this appears to be a Gamow-Teller allowed transition, it is not. In a simple shell model picture, the only beta transition to a $3 / 2$ state of seniority one transforms a P3/2 neutron into an F5/2 proton, which is 


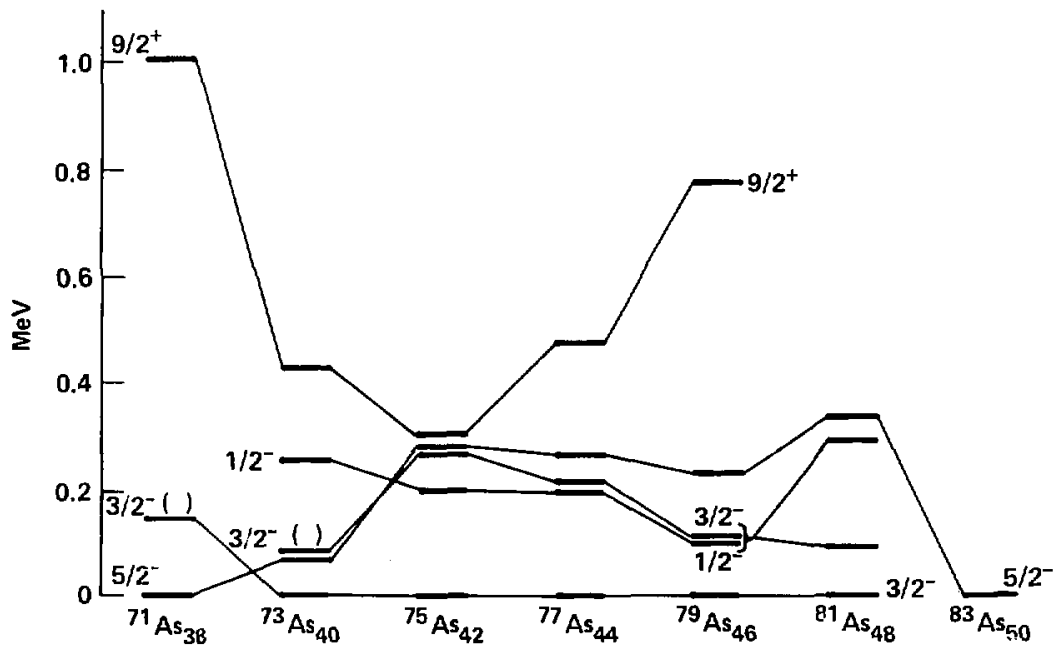

Fig. IV.3. Systematics of the nuclear levels of the odd-mass arsenics. The $5 / 2^{-}$level is the first excited state, but is low enough in energy that it could become the ground state in ${ }^{83} \mathrm{As}$ as it is in the light mass arsenics (HOF81).

an angular-momentum forbidden process. ] obtain a $\log (\mathrm{ft})=6.6$, which is consistent with other $\log (f t)$ value's for $15 / 2$ to $P 3 / 2 \beta^{-}$transitions (KON66).

I find no evidence for levels previously found at 0.36 and $0.43 \mathrm{MeV}$ (LIN65), in agreement with Montestruque et al. (MON78). Neither did I find any evidence tor the level at $0.822 \mathrm{MeV}$ (MON78) or $0.85 \mathrm{MeV}$ (LIN65). I give the arguments for assigning spin and parity to levels of ${ }^{83} \mathrm{Se}$ in Table IV.8. This completes the development of the ${ }^{83}$ As decay scheme which is presented in Fig. IV.A. 
Table IV.B. Spin and parity assignments. In this table the reasoning for the spin and parity assignments is presented. Where there is no discrepancy I took the spin and parity assignment from the ${ }^{82} \mathrm{Se}(\bar{d}, p)^{83}$ Se experiment of Montestruque et al. (MON78). The remainder of the assignments were made on the gamma-ray transitions and on the $\log \left(f_{0} t\right)$ values observed in this work. All observed gamma-ray transitions were assumed to be $E 1, M 1$, or $E 2$ transitions. The states with allowed beta transitions $\left(\log \left(f_{0} t\right) \leq 5.9\right)$ were restricted to possible spin and parity of $3 / 2,5 / 2$, and $7 / 2$. Spins are given as 2 ).

\begin{tabular}{|c|c|c|}
\hline $\begin{array}{l}\text { Energy } \\
\text { (keV) }\end{array}$ & $\begin{array}{l}\text { Spin, } \\
\text { Parity }\end{array}$ & Justification \\
\hline D & $9^{\prime}$ & MON78. \\
\hline 229 & 1 & MON78. \\
\hline 540 & $\mathbf{I}^{\prime}$ & MON38. \\
\hline 582 & $5^{*}$ & MON78. \\
\hline 963 & $\mathbf{3}$ & $\begin{array}{l}\text { Transitions to levels of spin and parity } 1 / 2^{\prime} \text { and } 1 / 2 \text { and transitions from levels of spin } \\
\text { and parity } 7 / 2 \text {. This assignment is consistent with levels in } \mathrm{Zr}_{44} \text { (NDS75) and } \mathrm{Sr}_{49} \text { (NDS79) }\end{array}$ \\
\hline 1063 & $15)$ & $\begin{array}{l}\text { where } 3 / 2 \text { levels have been identified near } 1 \text { MeV in transfer reaction studies. } \\
\text { Transitions to } 1 / 2 \text { and from } 7 / 2 \text { permit either } 3 / 2 \text { or } 5 / 2 \text {. The } 5 / 2 \text { assignment is } \\
\text { consistent with levels in } \mathrm{Zr}_{49} \text { and } 5 r_{49} \text { as in previous level. }\end{array}$ \\
\hline 1101 & 3 & MON78. \\
\hline 1265 & $17 \cdot 1$ & $\begin{array}{l}\text { Transitions to the } 3 / 2^{\circ}, 5 / 2^{\circ} \text {, and } 9 / 2^{\circ} \text { levels permit } 5 / 2^{\circ} \text { or } 7 / 2^{\circ} \text {. The lack of a transition } \\
\text { to the } 1 / 2^{\circ} \text { level suggests the assignment given. }\end{array}$ \\
\hline 1297 & $(11 \cdot)$ & $\begin{array}{l}\text { This assigninent is limited only by transition to the } 9 / 2 \text { ' level. However, the transitions } \\
\text { from the levels at } 1296,1331,1527 \text {, and } 1822 \mathrm{keV} \text { make it appear that they are four of the five } \\
\text { members of the one phonon } 9 / 2 \text { multiplet. Assuming this assignment, these are the only } \\
\text { reasonable spin and parities for these le "s!s. }\end{array}$ \\
\hline 1331 & $\mathbf{5}^{\prime}$ & MON78. \\
\hline 1473 & $(3)$ & $\begin{array}{l}3 / 2 \text { spin from gamsma-ray transitions. Posilive parity preferred from intensity of transitions } \\
\text { to positive parity states. }\end{array}$ \\
\hline 1526 & $19 \cdot 1$ & See the discussion for the level at $1297-\mathrm{keV}$. \\
\hline 1665 & $5^{\prime}$ & MON78. \\
\hline 1710 & $13 \cdot 1$ & $\begin{array}{l}\text { Transitions to } 1 / 2 \text { and } 5 / 2^{*} \text { Jevels permit } 1 / 2^{\prime}, 3 / 2 \text {, or } 5 / 2 \text { assignment. Transition inten- } \\
\text { sity to positive parity states favors the positive parity assignment. The } 3 / 2 \text { assignment is } \\
\text { consistent with a transition to the }(7 / 2) ; \text { level. }\end{array}$ \\
\hline 1822 & $(7)^{\circ}$ & $\begin{array}{l}\text { See the discussion for the level at } 1297-k e V \text {. Transitions limit the spin and parity to } 5 / 2{ }^{\circ} \text { or } \\
7 / 2 \text {. }\end{array}$ \\
\hline 1908 & 3,5 & Transitions to $1 / 2^{\prime}, 5 / 2^{\prime}$, and $5 / 2$ permit any of these assignments. \\
\hline 1943 & $5 \cdot .7$ & Transitions to $3 / 2$ and $9 / 2$ ' levels allow only these. \\
\hline 2077 & 7 & Transition to $9 / 2^{\prime}$ level and an allowed $\log (f t)=5.0$ give $7 / 2$ \\
\hline 2138 & $3^{1+1}$ & $\begin{array}{l}\text { Transitions to } 1 / 2,1 / 2,5 / 2 \text {, and } 5 / 2 \text { levels allowed only these assignments Presence in } \\
\text { Ref. MON78 suggests positive parity. }\end{array}$ \\
\hline 2190 & $5 \cdot .7$ & $\begin{array}{l}\text { Transitions to } 5 / 2 \text { and } 9 / 2{ }^{\circ} \text { levels and a transition from a } 3,5 / 2 \text { level allowed only these } \\
\text { assignments. Presence in Ref. MON78 reartion favors positive parity. }\end{array}$ \\
\hline 2482 & 5 & MON78. \\
\hline 2546 & $3^{*}$ & MON78, \\
\hline 2679 & 3 & $\begin{array}{l}\text { Transitions to levels of } 1 / 2^{\prime}, 1 / 2,5 / 2^{\prime} \text {, and } 5 / 2 \text { allowed a } 3 / 2 \text { assignment. Allowed } \\
\text { log(ft) }-5.9 \text { requires ncgative parity. }\end{array}$ \\
\hline 2725 & $5 \cdot$ & Transitions to $9 / 2,3 / 2{ }^{\circ}$ and $3 / 2$ allowed only the $5 / 2$ assignment. \\
\hline 2858 & 7 & Transition to $9 / 2^{\circ}$ level and the allowed $\left.\log (f)\right)-4.7$ permitted only the $7 / 2$ assignment. \\
\hline 2881 & (5) & $\begin{array}{l}\text { Transitions to } 3 / 2,3 / 2,5 / 2,5 / 2, \text { and } 7 / 2 \text { permitted } 3 / 2 \text { or } 5 / 2 \text { assignment. The } \\
\text { allowed log(fif })=4.7 \text { gives the negative parity assignment. The transition to the }(7 / 2 \%) \text { level } \\
\text { at } 1822 \mathrm{keV} \text { gives the }(5 / 2) \text { spin. }\end{array}$ \\
\hline 2471 & & Limited only by transitions to $1 / 2^{\prime}$ and $5 / 2$ levels, \\
\hline 2981 & 7 & Transition to $9 / 2$ level and an allowed $\log (f t)-5.1$ give the assignment. \\
\hline
\end{tabular}


Table IV.8. (Continued.)

\begin{tabular}{|c|c|c|}
\hline $\begin{array}{l}\text { Energy } \\
\text { (keV) }\end{array}$ & $\begin{array}{l}\text { Spin, } \\
\text { Parity }\end{array}$ & Justification \\
\hline 3167 & 3 & $\begin{array}{l}\text { Transitions to } 1 / 2,1 / 2^{+}, 5 / 2 \text {, and } 5 / 2^{+} \text {gave a spin of } 3 / 2 \text {. The allowed } \log (f t)=4.7 \text { gives } \\
\text { the negative parity. }\end{array}$ \\
\hline 3243 & 7 & Transition to $y / 2^{+}$level and the allo $N e d \log (f t)=4.7$ give this assignment. \\
\hline 3282 & 3 & $\begin{array}{l}\text { Transitions to } 1 / 2^{+}, 5 / 2^{+} \text {, and } 5 / 2^{-} \text {permitted } 3 / 2 \text { or } 5 / 2^{+} \text {assignment. The allowed } \\
\log (f t)=5.6 \text { gives the negative parity. }\end{array}$ \\
\hline 3334 & 3,5 & $\begin{array}{l}\text { Transitions } 103 / 2^{-}, 3 / 2^{*}, 5 / 2^{-}, 5 / 2^{+} \text {, and } 7 / 2^{-} \text {permit } 3 / 2^{-} \text {or } 5 / 2 \text {. The allowed } \log (f t)=5.6 \\
\text { gives the negalive parity. }\end{array}$ \\
\hline 3386 & & Restricted only by transition to $3 / 2 \%$ level. \\
\hline 3424 & & Restricted only by transitions to $3 / 2^{-}, 5 / 2^{+}$, and $5 / 2{ }^{-}$. \\
\hline 3464 & $5^{\prime}$ & MON78. \\
\hline 3558 & & Transition to $5 / 2^{+}$is the only restriction. \\
\hline 3690 & & Restricted only by transitions to $3 / 2^{-}$and $5 / 2^{-}$level 5 and allowed $\log (f t)=5.7$ \\
\hline 3828 & & Restricted only by a transitions to $3 / 2^{-}$and $5 / 2^{+}$leveis. \\
\hline 3911 & & Restricted only by a transition to $5 / 2^{+}$level. \\
\hline 4001 & & Restricted only by a transition to $3 / 2^{-}$level. \\
\hline
\end{tabular}




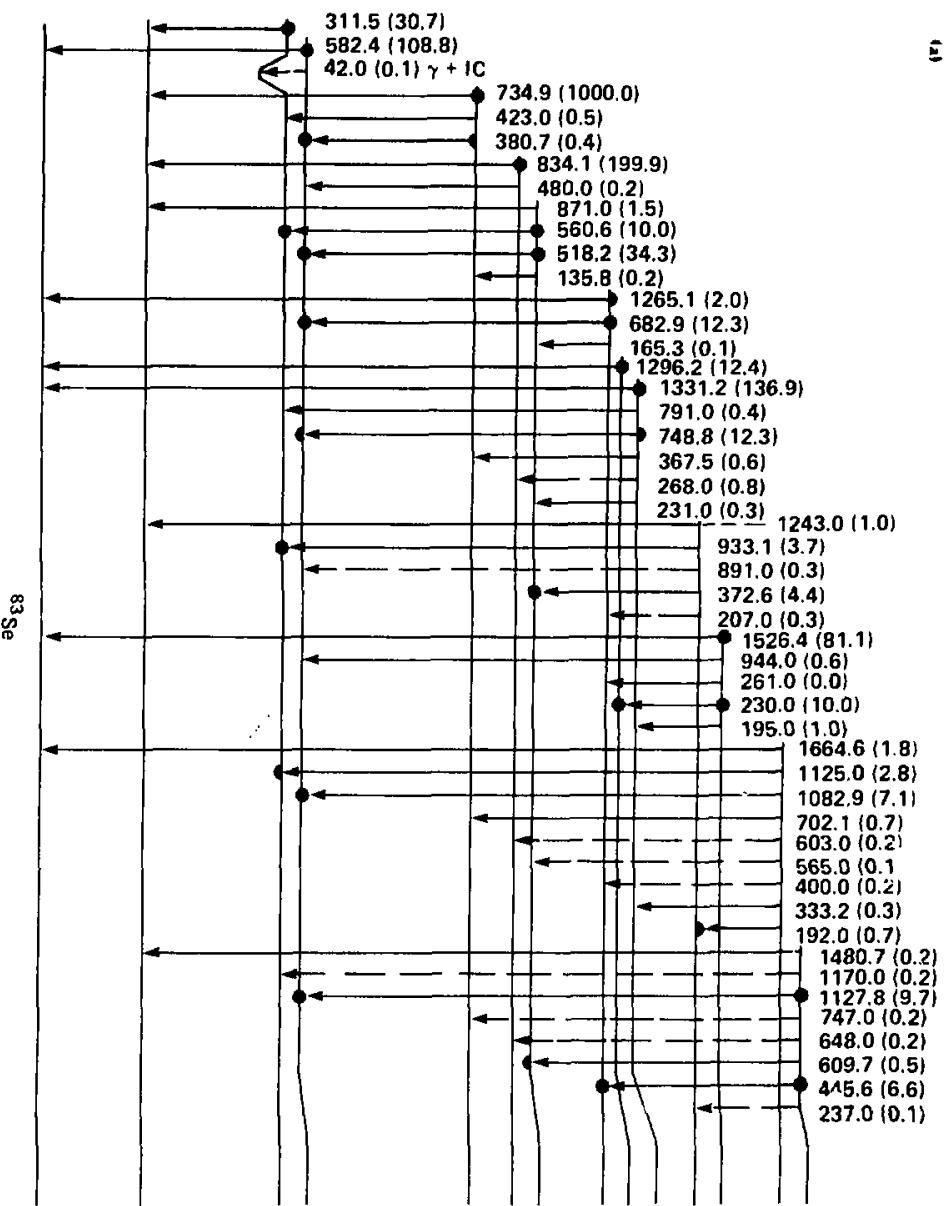

$\varliminf_{+} \quad \overrightarrow{N_{+}} \quad \vec{N}_{+}^{\frac{\pi}{N}}$

$\begin{array}{ll}2 & 0 \\ \vdots & \omega\end{array}$

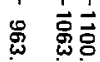

क

乔

芯芯

西

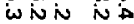

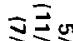

$\sum_{+} N_{+}^{N}$

$\bar{\omega} \underline{0}$

$\bar{N}_{+} \bar{N}_{+}$

NON

$\stackrel{i}{0}: 0$

or

\begin{tabular}{|c|c|c|c|c|c|}
\hline$\stackrel{0}{N}$ & $\stackrel{N}{\sigma}$ & $\overrightarrow{0}: 0$ & O & $\stackrel{0}{\mathrm{~N}}$ & 只 \\
\hline$+\infty$ & ه & c) 10 & 舟舟 & ن & in \\
\hline
\end{tabular}




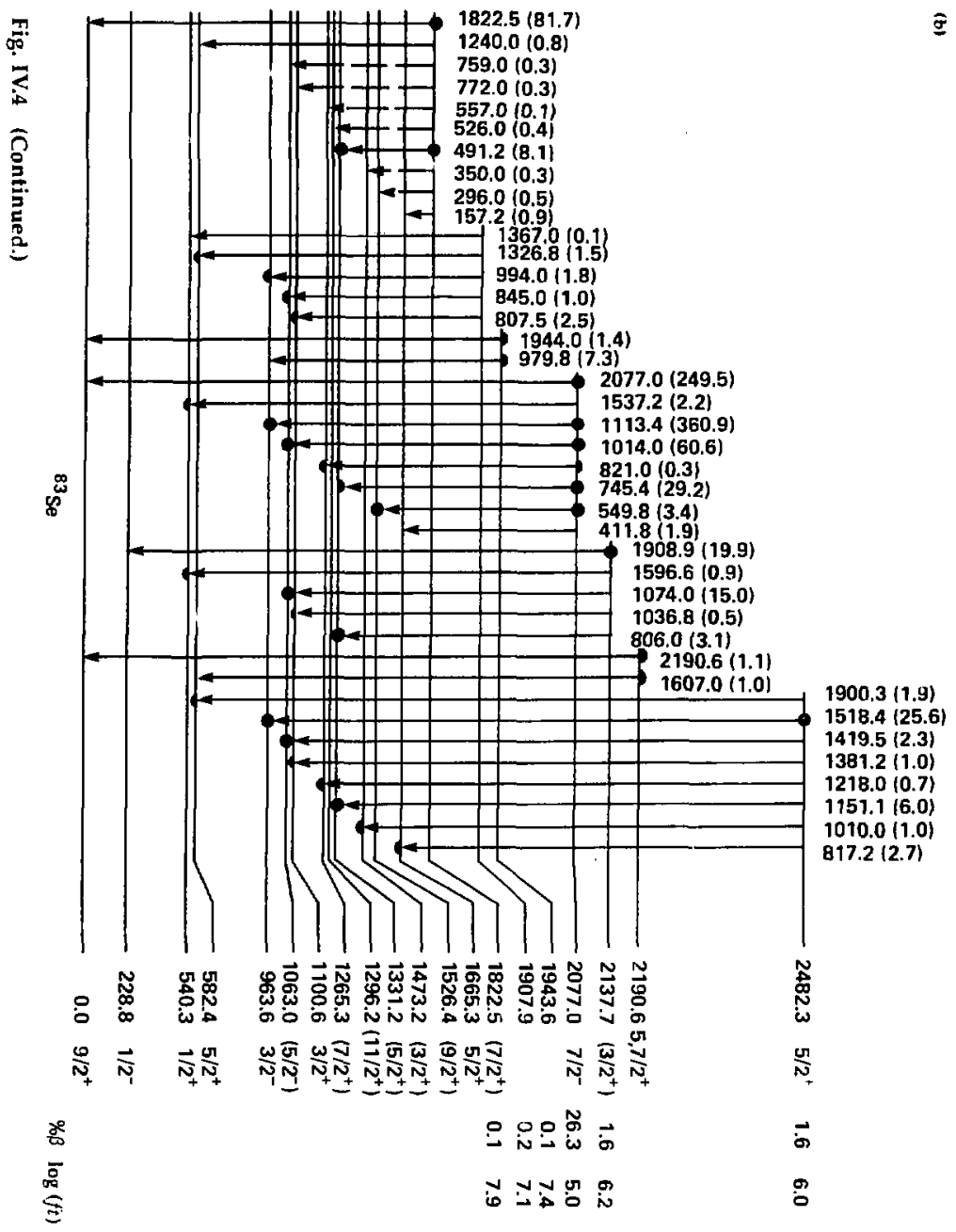


7
0
0
0
0
0
0
0
0
0
0

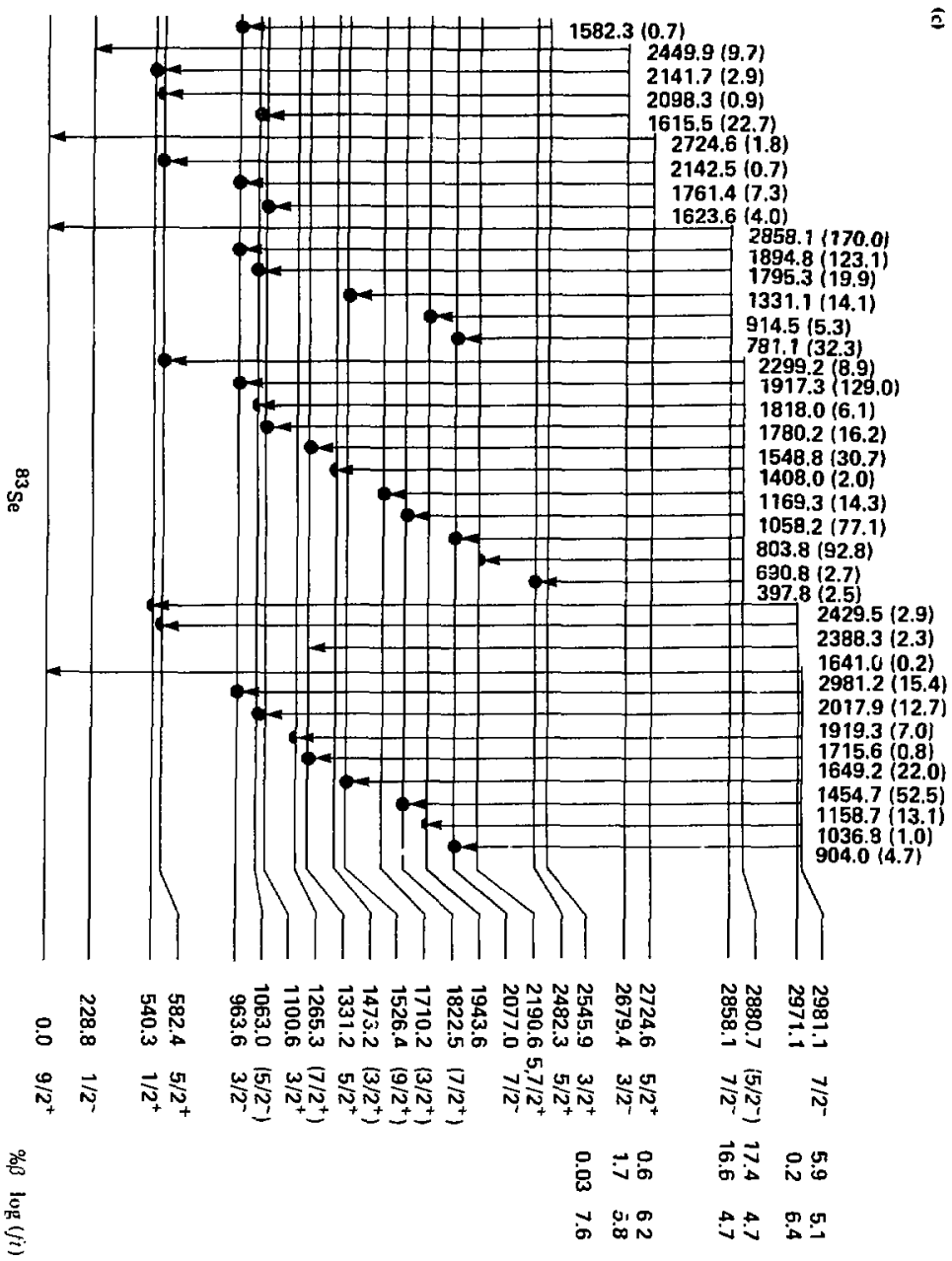




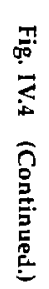

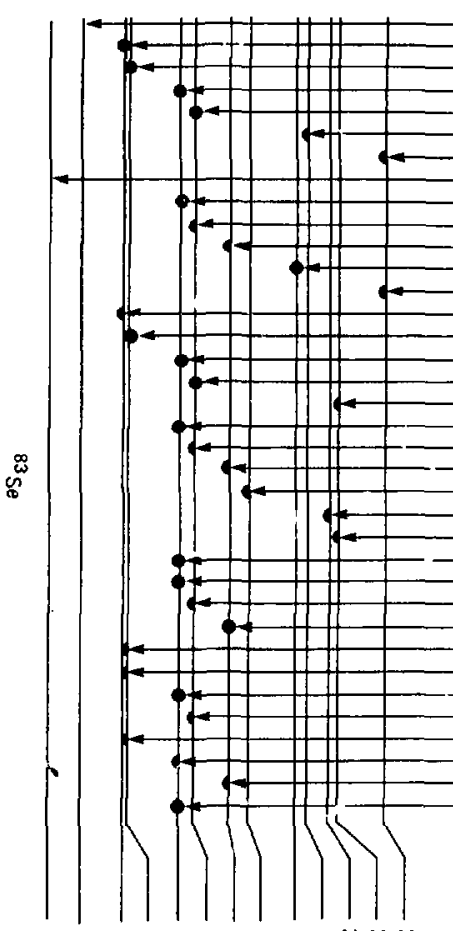

2937.9 (F.u)

$2629.0(0.3)$

$2585.2(1.7)$

2204.6 (213.4)

2104.2 (3.7)

1258.0 (1.9)

$685.0\{2.5\}$

3242.8 (35.1)

$2279.9(5.6)$

$2180.3(0.3)$

$1912.0(0.5)$

$1420.0(2.3)$

$760.6(1,4)$

2742.5 (4.4)

$2699.6(5.2)$

2318.8 (25.2)

2218.7 (14.4)

1143.6 (2.3)

$2370.4(11.4)$

$2270.8(1.5)$

$2001.0(1.2)$

$1860.0(1.7)$

1257.0 (2.3)

1.56 (1.4)

2423.1 (5.8)

2461.9 (2.5)

$2360.0(0.7)$

2091.0 (1.4)

$2881.4(1.1)$

$2976.2(1.1)$

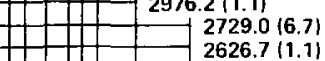

$1+3245.0(0.5)$

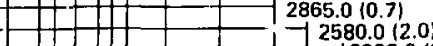

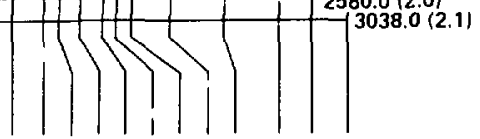

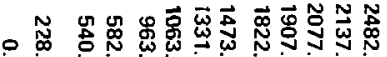

O

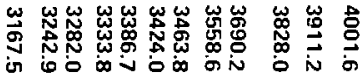

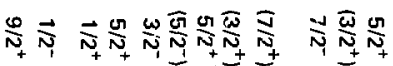

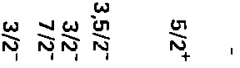

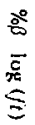

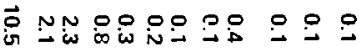

$\therefore$ W 


\section{Systematics of $N=49$ and $Z=49$ Nuclei}

In odd-mass nuclei lacking one proton (neutron) from shell closure and near mid neutron(proton-) shell there should be a set of levels based on a hole in the natural single proton (neutron) (th) states and another sot of levels, also at low energy, based on a particle in the intruder single proton (neutron) states and 2 holes in the natural single proton (neutron) states. These intruder states are found in the ${ }_{49} \mathrm{In}$ (i.e. $1 \mathrm{~g} 7 / 2,2 \mathrm{~d} 5 / 2,2 \mathrm{~d} 3 / 2$, and $3 \mathrm{~s} 1 / 2$ ) and ${ }_{81} \mathrm{Tl}$ (i.e. $1 \mathrm{~h} 9 / 2,1 \mathrm{i} 13 / 2$, and $267 / 2$ ) nudei (WOO80, ZGA80). Charge independence suggests that similar structure should occur in the $N=49$ and $N=81$ isotones, but this structure has not been observed. In particular, this structure has not been observed in ${ }^{\mathrm{K} / \mathrm{Z}} \mathrm{Zr}_{49}$ or ${ }^{87} \mathrm{Sr}_{49}$. However, I have observed this struclure in ${ }^{87}$ Se. Unified model calculations by Heyde have been used to completely describe the structure of the odd mass indium nuclei (HEY78, GLA79, HEY80 and earlier work described therein). I will use these unified model calculations performed by K. Heyde (HEY82a) to describe the structure of ${ }^{8.3} \mathrm{Se}_{49}$ and to describe the intruder level systematies for the $\mathrm{N}=49$ isotones.

In nuclei which lack a proton (neutron) from shell closure and are near mid neutron(proton-) sholl the following five significant properties are observed and serve to define the natural and intruder states:

- There is a group of states at low energy from across the shell closure, called intruder states.

- There exist vibrational bands built on both the natural and intruder states.

- The core for the intruder states is different from the core for the natural states.

- Camma-ray transitiuns between the natural and intruder states of the same parity are highly hindered.

- The Iransitions are all highly hindered.

I will discuss each of these propertips first for the indium $(Z=49)$ case which has been well developed and then for tie. ${ }^{37} \mathrm{Se}(\mathrm{N}=4 \mathrm{y})$ case to show the similarity.

The natural states for a single hole in either 50 nucleon shell are the $1 \mathrm{~g} 9 / 2,2 \mathrm{pl} / 2,2 \mathrm{p} 3 / 2$, and $165 / 2$ states. At low energy in the indium nuclei, levels have been identified through the ${ }^{\wedge} \mathrm{Cd}\left({ }^{3} \mathrm{He}, \mathrm{d}\right)^{\wedge} \cdot{ }^{1}$ In (A even) riactions which have large spectroscopic factors for the $\lg 7 / 2,2 \mathrm{~d} 5 / 2$, $2 d 3 / 2$ and $3 s 1 / 2$ single particle states (MAR74, THU70, HAR72); states which occur above the 50 particle shell closure. These levels would normally be expected to occur above $2 \mathrm{MeV}$ in excitation energy due to the single particle energy gap at the major shell closure. The systematics of these levels are shown in Fig. V.1. The intruder levels show a distinct minimum at ${ }^{117}$ In which is mid neutron-shell.

Other low-lying levels can be described by coupling the single particle or hole to vibrational modes of the core. The core for the intruder states is different from the core for the natural states. In a schematic sense the natural states are a "core + hole", while the intruder states are a "core + particle"; however the cores obviously do not have the same number of nucleons. The core for the natural states has 50 nucleons, while the core for the intruder states has 48 nucleons. The differences in the vibrational modes of these two cores can be seen by comparing the excitation encrgies of the first $2^{*}$ states in the even-even core nucleus. In Fig. V.2 these energies are shown for the even-even tin and cadmium isotopes $(Z=50$ and 48 , respectively). The excitation energy of the first $2^{\prime}$ state in the 48 particle core is approximately half that in the 50 particle core.

There exist vibrational bands built on both the natural and intruder states. These bands feature enhanced $M 1$ and $E 2$ intra-band transitions and highly hindered inter-band transitions. In the indium isotope's these enhancement factors have been measured (HEY78, GLA79, HEY80) anci show strongly enhanced $\mathrm{B}(l 2)$ values in the intruder band and enhanced $\mathrm{B}(M 1)$ from states in the virinity of the particle-one-phonon-vibrational multiplet built on the $9 / 2^{+}$hole. In ${ }^{8 .} \mathrm{Se}$, the measurements are not available for reduced transition probabilities, but the band structure is still apparent.

From the isospin independence of nuclear forces, states similar th those in $Z=49$ nuclei should occur in the $\mathrm{N}=49$ nuclei. Studies of ${ }^{\mathrm{N7}} \mathrm{Sr}$ and ${ }^{84} \mathrm{Zr}$ failed to find any of the particle intruder levels state below $2 \mathrm{MeV}$, except for the $2 \mathrm{~d} 5 / 2$. However, the ${ }^{82} \mathrm{Se}(\vec{d}, p)^{k .3}$ Se reaction study by 


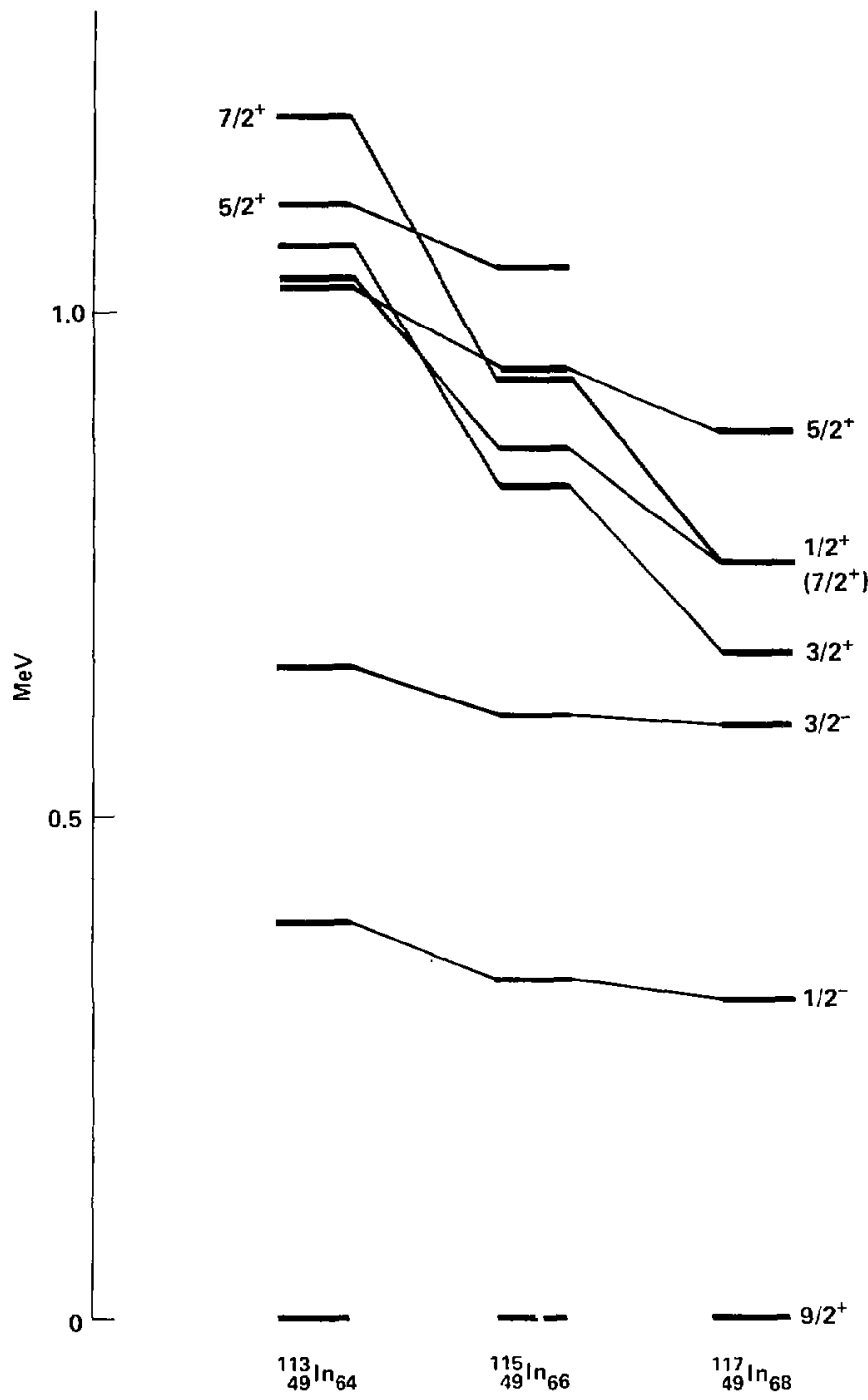

Fig. V.1. Systematics of the low lying levels in ${ }^{113-119}$ In with emphasis placed on the low lying $7 / 2^{+}, 5 / 2^{+}, 3 / 2^{+}$, and $1 / 2^{+}$intruder levels. 


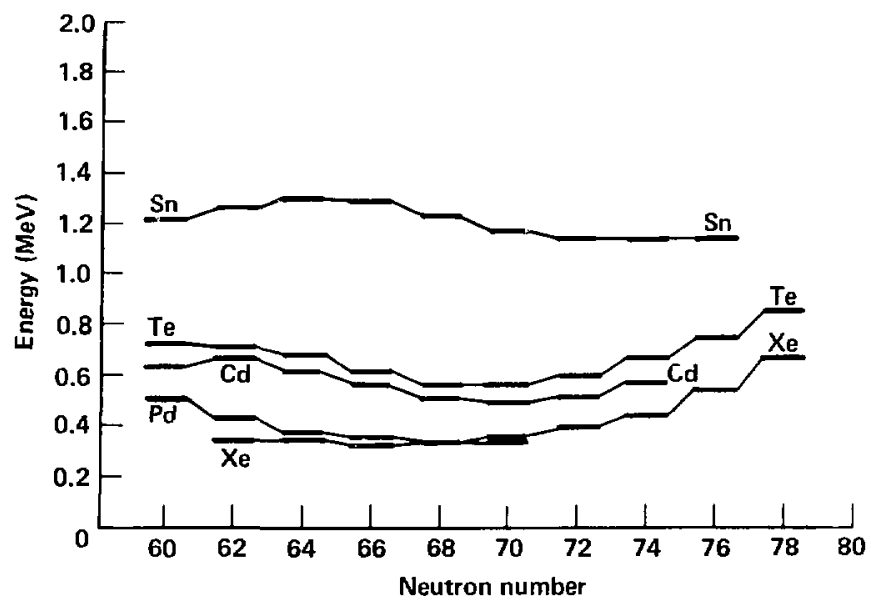

Fig. V.2. Systematics of the energy of the first $2^{+}$level in the indium region.

Montestruque, et al. (MON78) identified the intruder states $2 \mathrm{~d} 5 / 2,3 \mathrm{~s} 1 / 2$, and $2 \mathrm{~d} 3 / 2$ in ${ }^{4.3}$ Se at low energy. Figure V.3 shows a partial level diagram for the low energy intruder states relative to the natural states for selected $\mathrm{N}=49$ isotones.

The similarity of the intruder levels in the $N=49$ isotones is pointed up by the consistency of the spectroscopic factor, $(2]+1) S_{\mid l^{\prime}}$ for the $(d, p)$ reaction. These relatively constant values (Table V.1) for the $5 / 2^{4}, 1 / 2^{*}$, and $3 / 2^{+}$levels for ${ }^{83} \mathrm{Se},{ }^{85} \mathrm{Kr}$ and ${ }^{87} \mathrm{Sr}$ show that while the levels move up in excitation energy, the single particle component is relatively constant.

However, in the neutron case, the intruder band structure is well developed only in ${ }^{k .3}$ Se and ${ }^{81} \mathrm{Ge}$, where the energy of the first $2^{+}$state in the $\mathrm{N}=48$ is below $0.7 \mathrm{MeV}$ (Fig. V.4). This is the energy of the first $2^{+}$in the cadmium isotopes. I will discuss this topic further when comparing the theoretical and experimental results.

The unified model describes most of the $Z=49$ (indium) isotopes very well, particularly 113.115,117,119 In (HEY78, GLA79, HEY80 and earlier work described therein). Attempts to use a similar description in the $\mathrm{N}=49$ nucle ${ }^{89} \mathrm{Zr}$ and ${ }^{87} \mathrm{Sr}$ have not met with success. I will compare the properties of these two groups of nuclei to show that this form of the vibrational model does discribe the $\mathrm{N}=49$ nucleus, ${ }^{\text {Bi. }} \mathrm{Se}$.

Table V.1. Systematics of the spectroscopic factor for the $(\mathrm{d}, \mathrm{p})$ reaction to produce ${ }^{83} \mathrm{Se},{ }^{85} \mathrm{Kr}$ and ${ }^{87} \mathrm{Sr}$. The values given are for (2J+1)S with the level energy in $\mathrm{MeV}$ in parentheses.

\begin{tabular}{|c|c|c|c|}
\hline \multirow{2}{*}{$\begin{array}{c}\text { Spin } \\
\text { Parity }\end{array}$} & \multicolumn{3}{|c|}{ Naleus } \\
\hline & ${ }^{63} 5 e$ & $\mathrm{Kr}$ & ${ }^{81} \mathrm{St}$ \\
\hline $5 / 2$ & $2.76(0.58)$ & $3.43(1.14)$ & $2.94(1.78)$ \\
\hline $3 / 2$ & $0.60(1.10)$ & $0.36(2.06)$ & $0.35(2.7)$ \\
\hline $1 / 2^{\prime}$ & $0.56(0.54)$ & $0.76(1.43)$ & $0.56(2.18)$ \\
\hline
\end{tabular}




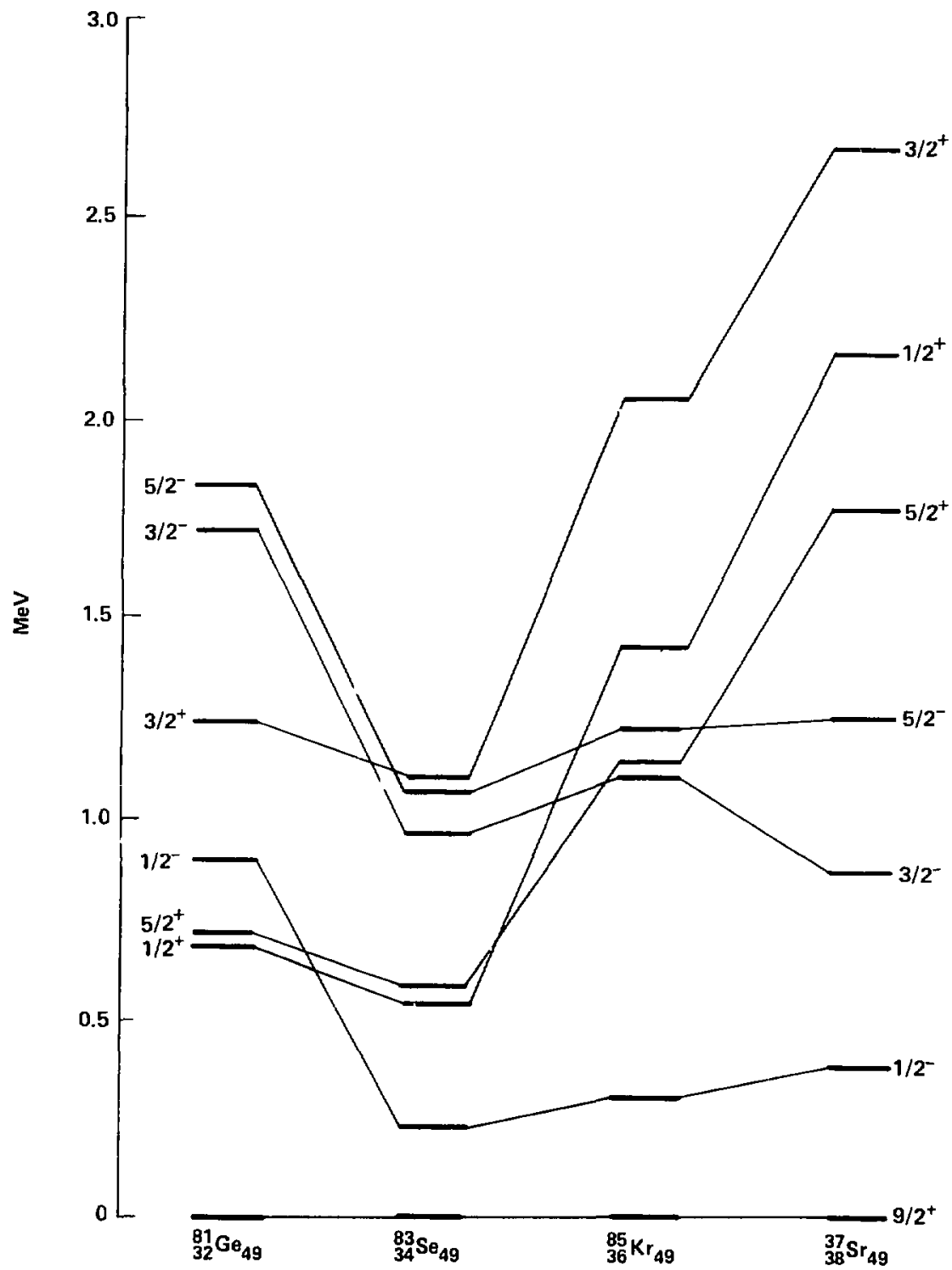

Fig. V.3. Systematics of the low lying levels in the $\mathrm{N}=49$ isotones, with emphasis placed on the low-lying $5 / 2^{+}, 1 / 2^{+}$, and $3 / 2^{+}$intruder levels. 


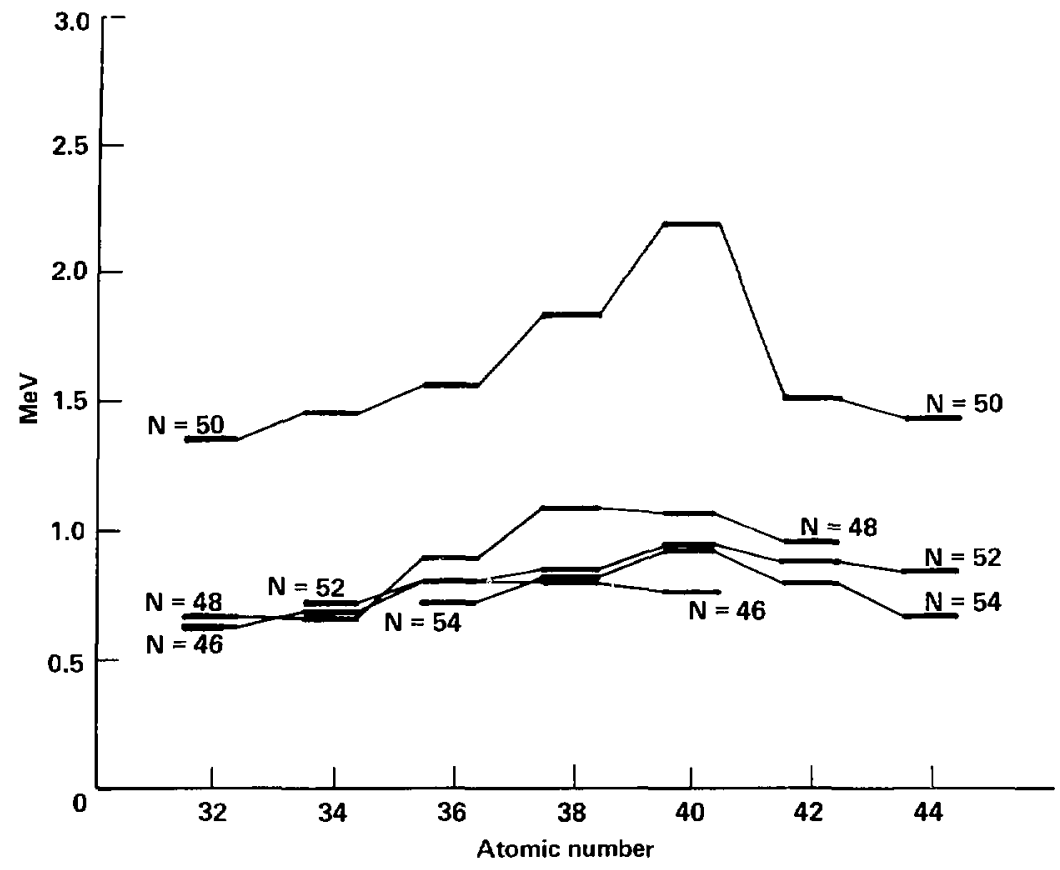

Fig. V.4. Systematics of the energy of the first $?^{+}$level in the $\mathrm{N}=50$ region.

\section{Theory}

The unified model is a phenomenological model for describing collective core excitations and the coupling of these core excitations to the valence particle(s). In the $N=49$ and $Z=49$ nuclei these core excitations are vibrations. I will give a qualitative description of the vibrations of the even-even core, followed by the odd-even case in the weak coupling linit. The unified model then introduces the more realistic intermediate coupling. I will discuss the unified model as developed by Heyde and others to explain the structure observed in indium nuclei, and use this model to explain the structures of ${ }^{33}$ Se and other $N=49$ isotones.

\section{Vibrational Model}

The vibrations will appear directly as part of the low energy $(<2.5 \mathrm{MeV})$ nuclear structure only if the energy required to excite them is less than approximately $2.5 \mathrm{MeV}$. Thus, monopole and dipole vibrations are not observed at low energy. The monopole vibration occurs at high excitation energy because the nuclear density is fixed. The dipole vibration can appear only as the giant dipole resonance which occurs at excitation energies above $15 \mathrm{MeV}$. I will restrict the discussion to quadrupole vibrations. Higher order vibrations (particularly the $3^{-}$, ortupole vibration) 
have been observed. They are treated similarly to the quadrupole vibrations and can couple to other vibrations and the single particles and holes. They will not be discussed further as the octupole vibration lies near $\mathrm{JMV}$ in $\mathrm{N}=48$ and $\mathrm{N}=50$ nuclei (MAT77), while the quadrupole vibration occurs at 0.7 and $1.5 \mathrm{MeV}$, respectively, in these nuclei.

Vibrational structure appears as collective motion in even-even nuclei. In the weak coupling limit there is no interaction between the different vibrational levels. The Hamiltonian is (BOH75)

$H_{\text {coll }}=\sum b^{\dagger} b$

where $b^{\prime}$ is the creation operator for vibrational quanta (phonons). The levels obtained for quadrupole vibration are shown in column 2 of Fig. V1.1. Each level can be identified by $(n, R)$ where $\|$ is the number of phonons which are coupled to angular momentum $R$. The levels are at an excitation energy given by $\mathrm{E}_{u}=n h \omega$, where $h \omega$ is the phonon energy. The levels are degenarate in $R$.

When an odd particle (hole) of spin and parity $j$ is added to the even-even vibrational core the Hamiltonian becomes (BOH75)

$H=H_{\text {rull }}+H_{\mathrm{s}}$

where $H_{4}$ describes the single particle motion. The levels are obtained at the same excitation energies as in the even-even case but the states are now described as $[(n, R) i] /$ where the single particle of yin and parity $i$ coupled to the core angular momentum, $R$, to give total angular momentum and parity /, with / restricted by

$R+1>1 \geq|R-j|$.

These levels are also at excitation energy $\mathrm{E}_{\mu}=n h \omega$ and are degenerate in both $R$ and $/$. The spins for a $1 / 2^{\prime}$ and a $9 / 2$ 'particle are shown in columns 4 and 5 of Fig. VI.1, respectively.

The degeneracy can be removed by adding to the Hamiltonian a residual interaction term which treats the particle-phonon interaction,

$H_{1 \mathrm{rot}}-\lambda \sum_{\mu}^{\prime} b_{2 \mu} Y_{2 \mu} \times$

\begin{tabular}{|c|c|c|c|c|}
\hline 3 & $0,2,3,4,6$ & $\longrightarrow$ & $\begin{array}{l}1: 3,5 ; 5,7 ; 7 \\
9 ; 11,13\end{array}$ & $\begin{array}{l}9 ; 5,7,9,11,13 ; 3,5,7,9,11,13 \\
15 ; 1,3,5,7,9,11,13,15,17 ; 1,3 \\
5,7,9,11,13,15,17,19,21\end{array}$ \\
\hline 2 & $0,2,4$ & - & $1,3,5,7,9$ & $\begin{array}{l}9 ; 5,7,9,11,13 ; 1,3,5,7,9,11,13 \\
15,17\end{array}$ \\
\hline 1 & 2 & & 3,5 & $5,7,9,11,13$ \\
\hline ) & 0 & $-\ldots$ & 1 & 9 \\
\hline & $\mathbf{R}$ & Jevel & $j=1 / 2$ & $j=9 / 2$ \\
\hline
\end{tabular}

Fig. VI.1. Energy levels for quadrupole vibration. Spins are given for quadrupole vibratiou, column 2, and for quadrupole vibı ation coupled to a spin $1 / 2$ particle, column 4 , and for quadrupole vibration coupled to a spi $9 / 2$ particle, column 5 . For the odd particle the spins are given as 2J. 
where $Y$ is the spherical harmonics and $x$ is the particle coordinates. The effect of varying the coupling constant, $k$, in the case of one phonon and a $5 / 2^{+}$particle has been discussed by Choudhury (CHO54).

The observables of interest are the evel energies, the reduced transition probabilities, and the spectroscopic factor for particle stripping reactions. The electromagnetic transitions can go only by $E 2$ (for quadrupole vibration) or $M 1$ radiation. For $E 2$ transitions the selection rule is

$\left|n_{i}-n_{f}\right|=1$

The reduced transition probabilities for transitions between multiple phonon states for quadrupole vibrations are given by (BOH75,p. 360)

$\left.B|E 2 ;|(n, R) j^{\prime} i \rightarrow \rightarrow\left|\left(n^{\prime}, R^{\prime}|i| I^{\prime} \mid=(2)^{\prime}+1\right)\right| 2 R+1\right) \times\left\{\begin{array}{lll}R & j & J \\ J^{\prime} & 2 & R^{\prime}\end{array}\right\}^{2} B\left(E 2 ;(\mathrm{n}, R) \rightarrow\left(n^{\prime}, R^{\prime} \|\right.\right.$

where the only transitions have multipolarity E2. For M1 transitions the matrix element is (BOH75,p. 360)

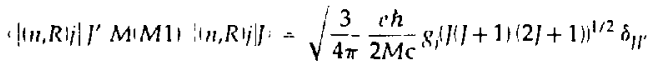

$$
\begin{aligned}
& +(-1)^{R+1+1+1}\left(\left(g_{\lambda}-g\right) R(R+1)(2 R+1)\right)^{1 / 2}\left\{\begin{array}{lll}
R & j & l \\
\gamma^{\prime} & 1 & R
\end{array}\right\} .
\end{aligned}
$$

The spectroscopic factor for transfer reactions can be obtained. If the state is expanded as

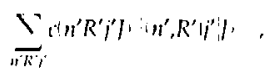

then the spectroscopic factor is given by

$S_{0,}=c^{2} !(0), p|j|$

for the transfer of a particle of spin and parity $j$ into the even-even core with spin and parity $0^{\prime}$.

\section{Unified Model}

The coexistence of the natural and intruder levels requires a model that treats both types of levels on an equal footing. The unified model has heen extended to do this by K. Heyde (HEY78, HEY 80). For completeness I describe this model in the following section.

The Hamiltonian must be separable as

$$
H=H_{\text {cull }}+H_{4 l^{\prime}}+H_{111}
$$

where the three paris of the Hamiltonian describe the collective, single particle and residual interaction. The actual Hamiltonian is given by

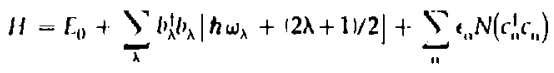




$$
\begin{aligned}
& +\sum_{\alpha, \beta \lambda \mu}\left\langle\alpha\left|\Upsilon_{\lambda \mu}\right| \beta\right) h \omega_{\lambda} E_{\lambda}\left[b_{\lambda \mu}^{\dagger}+\left(-1 \mu b_{\lambda \mu}\right] N\left(c_{\alpha}^{\dagger} c_{\beta}\right)\right.
\end{aligned}
$$

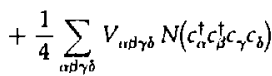

where $E_{n}$ is the energy of the $0^{+}$ground state in the $N=50$ nucleus.

$b_{\lambda \mu}^{\prime}$ is the phonon creation operator, with the restriction $\lambda=2$

$h \omega_{\lambda}$ is the phonon energy in the $N=50$ nucleus

$\epsilon_{0}$ is the single particle energy

$E_{\lambda}$ is the dimensionless coupling coefficient, defined by

$$
L_{2} h \omega_{2}=\left\langle\frac{\partial V}{\partial r}\right) \frac{4\left(5 \pi \pi^{1 / 2}\right.}{3 Z r^{2} R_{0}^{2}} B\left(E 2 ; 2_{1}^{+} \rightarrow O_{1}^{+}\right)^{1 / 2}
$$

$N(c, \ldots)$ is the number operator

$V_{\ldots, \gamma^{\prime}}$ is the residual interaction hetween the neutron particles or holes.

By restricting the single particle degrees of freedom to the $2 \mathrm{~d} 5 / 2,3 \mathrm{~s} 1 / 2,2 \mathrm{~d} 3 / 2,1 \mathrm{~g} 7 / 2$, and $1 \mathrm{~h} 11 / 2$, and the single hole degrees of freedom to the $1 \mathrm{~g} 9 / 2,2 \mathrm{p} 1 / 2,2 \mathrm{p} 3 / 2$, and $1 \mathrm{f5} / 2$ orbitals the Hamiltonian can be restructured as

$H=H_{\mathrm{s}}+H_{\mathrm{pl}}+H_{\mathrm{hh}}+H_{\mathrm{ph}}+H_{\mathrm{hc}}+H_{\mathrm{phc}}+V_{\mathrm{hh}}+V_{\mathrm{ph}}$

where 11 : describes the low-lying excitations of the $\mathrm{N}=50$ core,

$H_{4}, H_{\mathrm{H}} \quad$; describe the single-particle and thole motion,

$H_{1}, H_{\mathrm{h}}, H_{1 \mathrm{lu}}:$ describe the particle-, hole- and particle-hole-core interaction,

$l_{\mathrm{hb}}, \mathrm{l}_{\mathrm{ph}} ;$ describe the residual hole-hole and particle-hole interaction,

II. $-I_{11}+\sum_{\lambda} b_{\lambda} b_{\lambda} \mid h \omega_{\lambda}+[2 \lambda+1 / 2 \mid$ :

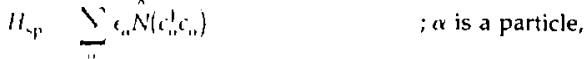

$H_{h}-\sum_{n} c_{n} N\left(i_{n} i_{n}\right) \quad ; \alpha$ is a hole,

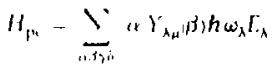

$\times\left|b_{\lambda_{\mu}}^{1}+1 \cdot 1^{\mu} b_{\lambda_{,}}\right| N\left(c_{n}^{-1} c_{n}\right) \quad ; \alpha, \beta$ are particles,

$H_{1,}$. $\quad$ " $\alpha, \beta$ are holes

$H_{1, h}$ - $\quad \quad \quad ; \alpha$ is a particle and $\beta$ is a hole or the reverse,

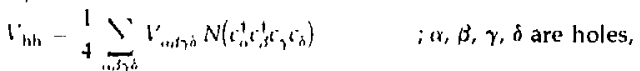

$l^{\prime h+}=$

$; \alpha, \beta, \gamma, \delta$ are particles or holes; $\alpha, \beta$ different and $\gamma, \delta$ different.

The allowed configurations are one hole and one particle plus two holes.

The first step in solving the Hamiltonian, Eq. (VI.13), is to describe the low'-lying excitations in the $N-48$ isolones

$\left.\left(H_{t+1} N-4\right)-\omega N-4\right)$ 
which is explicitly

$\left.\left.\left(H_{\mathrm{s}}+H_{\mathrm{sh}}+H_{\mathrm{hc}}+V_{\mathrm{lh}}\right) \mid \mathrm{N}=48 ; I^{\prime \prime \prime} M\right)=\omega(l, i) \mid \mathrm{N}=48 ; I^{\prime \prime \prime} M\right)$

where the $N=48$ eigenstates are expanded to

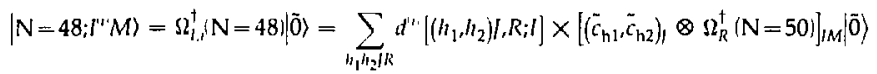

where:

(1) is the ith state with spin and parity 1 ,

$\Omega^{1} \quad$ is the phonon creation operator in the appropriate nucleus,

$\left.d^{\prime \prime \prime} l ..\right)$ is the expansion coefficient for the state having holes in single particle states $h_{1}$ and $h_{2}$ coupled to / total angular momentum; the single particle state is then coupled to the $\mathrm{N}=50$ phonon state. $\mathrm{n}$ phonons coupled to to angular momentum $R$, which are finally coupled to total angular momentum state I with $\mathrm{z}$ component $M$,

$i_{h} \quad$ is the annihilation operator, which creates holes,

$R$ abbreviates $\left(n_{\lambda}, R_{\lambda}\right) R$.

Solution of Eq. (VI.16) yields the phonon states for $N=48$.

Having obtained the phonon levels for $N=48$ and $N=50$ nuclei, the states of the $N=49$ nucleus are created. For the hole states in the $\mathrm{N}=50$ core

$\left|i_{h}{ }^{\prime}, N=50(R ! ; M)=\right| \tilde{c}_{1} \otimes \Omega R_{k}\left(N=\left.50 i\right|_{\mid M}|\tilde{0}\rangle\right.$

and for the particle states in the $N=48$ core

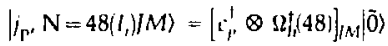

The resulting wave functions are then (in the most gencral form)

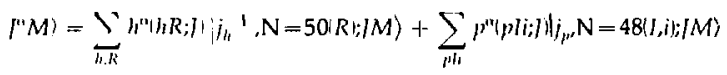

The secular equations for the $N=49$ nuclei were then expressed in matrix form as

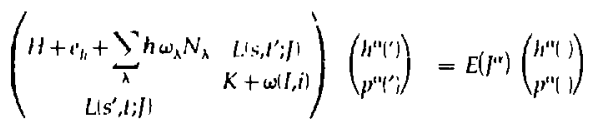

where

$$
\begin{aligned}
& H=H^{\prime}\left(h R, h^{\prime} R^{\prime} ;\right) \\
& =\left\langle i_{h}{ }^{1}, \mathrm{~N}=50(R)_{i} J\left|V_{\mathrm{hc}}\right| j_{h^{\prime}}{ }^{-1}, \mathrm{~N}=50\left(R^{\prime}\right)_{i}\right\rangle \\
& L\left(s, t^{\prime} ; I\right)=\lambda\left(h R, f^{\prime} l^{\prime} i^{\prime} ;\right) \\
& =\left\langle j_{h}{ }^{\prime}, \mathrm{N}=50(R)_{i} ;\left|V_{\mathrm{ph}}+H_{\mathrm{phc}}\right| j_{p}, \mathrm{~N}=48\left(V^{\prime} i^{\prime}\right) ;\right\rangle \\
& L\left(s^{\prime}, t ;\right)=\lambda\left(h^{\prime} R^{\prime}, p / i ;\right) \\
& \left.K=K\left(p l i, p^{\prime} l^{\prime} i^{\prime} ;\right]\right) \\
& =\left\langle j_{r}, \mathrm{~N}=48(l i)_{;} /\left|V_{\mathrm{ph}}+H_{\mathrm{rc}}\right| j_{p^{\prime},}, \mathrm{N}=48\left(l^{\prime} i^{\prime}\right) ; J\right\rangle \\
& h^{\alpha}\left({ }^{\prime}\right) \quad=h^{\prime \prime \prime}\left(h^{\prime} R^{\prime} ;\right) \\
& h^{\prime \prime}()=h^{\prime \prime}(h R ; I)
\end{aligned}
$$




$$
\begin{aligned}
& p^{\prime \prime \prime}\left({ }^{\prime}\right)=p^{\prime \prime}\left(p^{\prime} l^{\prime} i_{i} ;\right) \\
& \left.p^{\prime \prime}()=p^{\prime \prime}\left(p / i_{i}\right)\right)
\end{aligned}
$$

Detailed expansions for these matrix elements are given in Appendix A of reference HEY78.

\section{Parameters}

I now give the parameters used to describe ${ }^{83} \mathrm{Se}$ (HEY82). The collective parameters are

$h \omega_{2}(\mathrm{~N}=50)=1.4 \mathrm{MeV}$

$h \omega_{2}(\mathrm{~N}=48)=0.655 \mathrm{MeV}$

from experimental measurement for the quadrupole phonon energies. No octupole phonons were used as these have been identified at $2.5 \mathrm{MeV}$ (MAT77) for nuclei with $\mathrm{N}<51$ and $\mathrm{Z}>30$ and will have little effect on the low energy structure. Values for the dimensionless coupling coefficients are

$l_{2}(\mathrm{~N}=50)=1.0$

$\Gamma_{2}(\mathrm{~N}=48)=6.0$,

which are taken from Eq. (VI.12) using experimental values for $\hbar \omega_{2}$ and B(E2) with slight empirical adjustment. The single particle and hole energies in $\mathrm{MeV}$ are

$\begin{array}{llll}\epsilon_{1 \mathrm{~h} 11 / 2} & 2.6 & & \\ \epsilon_{\mathrm{lg} 7 / 2} & 2.8 & \epsilon_{1 \mathrm{r} / 2} & 1.35 \\ \epsilon_{2 \mathrm{~d} 3 / 2} & 2.6 & \xi_{2 \mathrm{p} 3 / 2} & 1.15 \\ \epsilon_{3 \mathrm{~s} 1 / 2} & 1.5 & \epsilon_{2 \mathrm{pl} / 2} & 0.40 \\ \epsilon_{2 \mathrm{~d} 5 / 2} & 0 & \epsilon_{1 \mathrm{~g} 9 / 2} & 0\end{array}$

which were modified from Ref. REE70. The energy required to create a particle hole pair, $\mathrm{E}_{\mathrm{ph}}$, is $2.15 \mathrm{MeV}$. The residual interaction, $V_{\text {udd }}$, was a delta function force without spin exchange of the form $V=V_{0} \delta\left(r_{1}-r_{2}\right)$ witin $V_{0}$ adjusted to fit the levels of ${ }^{B 2} \mathrm{Se}$ (HEYBO).

I compare the results of the model calculations to three sets of experimental data: the energy levels, the gamma-ray transition data from the decay of ${ }^{83} \mathrm{AS}$ and the ${ }^{82} \mathrm{Se}(\overrightarrow{\mathrm{d}}, \mathrm{p}){ }^{83} \mathrm{Se}$ stripping reaction. The operator for the electric moments is

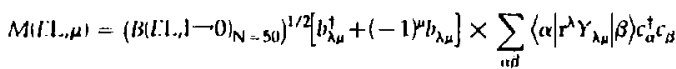

and for the magnetic dipole moment is

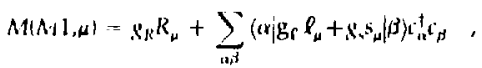

where sf and $s$, are the orbital and spin gyromagnetic factors, respectively. The detailed matrix elements that result from these operators are given in Appendix B of Ref. HEY78. The transitions can be reduced to three pertinent transition types:

1. Single particle transitions within either the $N=4 B$ or $N=50$ subsystem.

2. Collective transitions in either the $N=48$ or $N=50$ subsystem.

3. Single particle transitions between the $N=48$ and $N=50$ subsystems. 
With these operators level lifetimes, M1-E2 mixing ratios and branching ratios for the levels can be calculated.

The spectroscopic factors for neutron stripping into levels above the $\mathrm{N}=50$ shell closure is

$S_{\hat{h}}^{\prime \prime}(j)=p^{\prime \prime}\left(\ell ;, 0_{1}^{\prime} ; j\right)^{2} \delta_{j}$

These operators allow calculation of the experimentally observed values in ${ }^{83} \mathrm{Se}$. The other operators applicable to this model are described in Ref. HEY78. They include operators for the neutron pickup reaction, and the static magnetic dipole and electric quadrupole moments.

\section{Discussion}

I will compare first the theoretical and experimental results for ${ }_{34}^{83} \mathrm{Se}_{49}$; the level properties, spin and parity, excitation energy, and spectroscopic factor. The theory is the unified model discussed in the previous section and the, experimental measurements are my gamma-ray measurements and the ${ }^{82} \mathrm{Se}(\overrightarrow{\mathrm{d}}, \mathrm{p})^{83}$ Se experiment by Montestruque et al. (MON78). Next, I will compare the observed and predicted gamma-ray transitions in ${ }^{83}$ Se. Last, I will discuss the effect of $Z=40$ shell closure, which blocks the collectivity needed to develop the intruder structure levels at low energy in nuclei near $Z=40$.

I expect three types of levels in ${ }^{83}$ Se: the natural, negative parity levels arising from the $2 \mathrm{p} 1 / 2$, $2 \mathrm{p} 3 / 2$, and $185 / 2$ single neutron hole states; the natural, positive parity levels arising from the $1 \mathrm{~g} 9 / 2$ single neutron hole states; and the intruder, positive parity levels arising from the $2 \mathrm{~d} 5 / 2$, $2 \mathrm{~d} 3 / 2,3 \mathrm{~s} 1 / 2$, and $\mathrm{lg} 7 / 2$ single particle states. The model $1 \mathrm{~h} 11 / 2$ component does not mix with any of the natural components, nor does it occur in any levels below $3.6 \mathrm{MeV}$. The parameters used in modeling ${ }^{83}$ Se were given in the previous section. In Appendix 3 , I give the theoretical composition of the levels to $3.5 \mathrm{MeV}$ and up to spin $13 / 2^{+}$and $7 / 2^{-}$. In Fig. VIl.1, I show a ladder diagram comparing theoretical and observed energies. A fourth type of level has been added; those positive parity levels where neithur natural or intruder dominates substantially.

\section{Natural Levels}

The first $1 / 2^{-}, 3 / 2^{-}$, and $5 / 2^{-}$levels show a good comparison in energy between theory and experiment. The theoretical group of levels between 1.7 and $2.0 \mathrm{MeV}$ is not clearly identified experimentally and may be the levels of uncertain parity indicated in Fig. VIl.1. The gamma-ray transition branching ratios will be discussed later.

The $\left(11 / 2^{+}\right), 5 / 2^{+},\left(9 / 2^{+}\right)$and $\left(7 / 2^{+}\right)$levels at $1296,1331,1527$, and $1822 \mathrm{keV}$ could be members of the $\left[(1,2) 9 / 2^{+}\right]$multiplet. Theoretically the multiplet is rather unmixed with the multiplet component being at least 0.8 in all levels. Level energies show good agreement between theory and experiment as shown in Fig. VII.1. For an unmixed multiplet, the energy centroid, given by

$\sum_{1} E_{1} / 5$

should be the energy of the first $2^{+}$state in the core nucleus, ${ }^{84} \mathrm{Se}$, which is at $1454 \mathrm{keV}$. For the four levels observed, the centroid is at $1494 \mathrm{keV}$, experimentally, and $1476 \mathrm{keV}$, theoretically. For the complete multiplet the centroid is at $1454 \mathrm{keV}$, theoretically. 


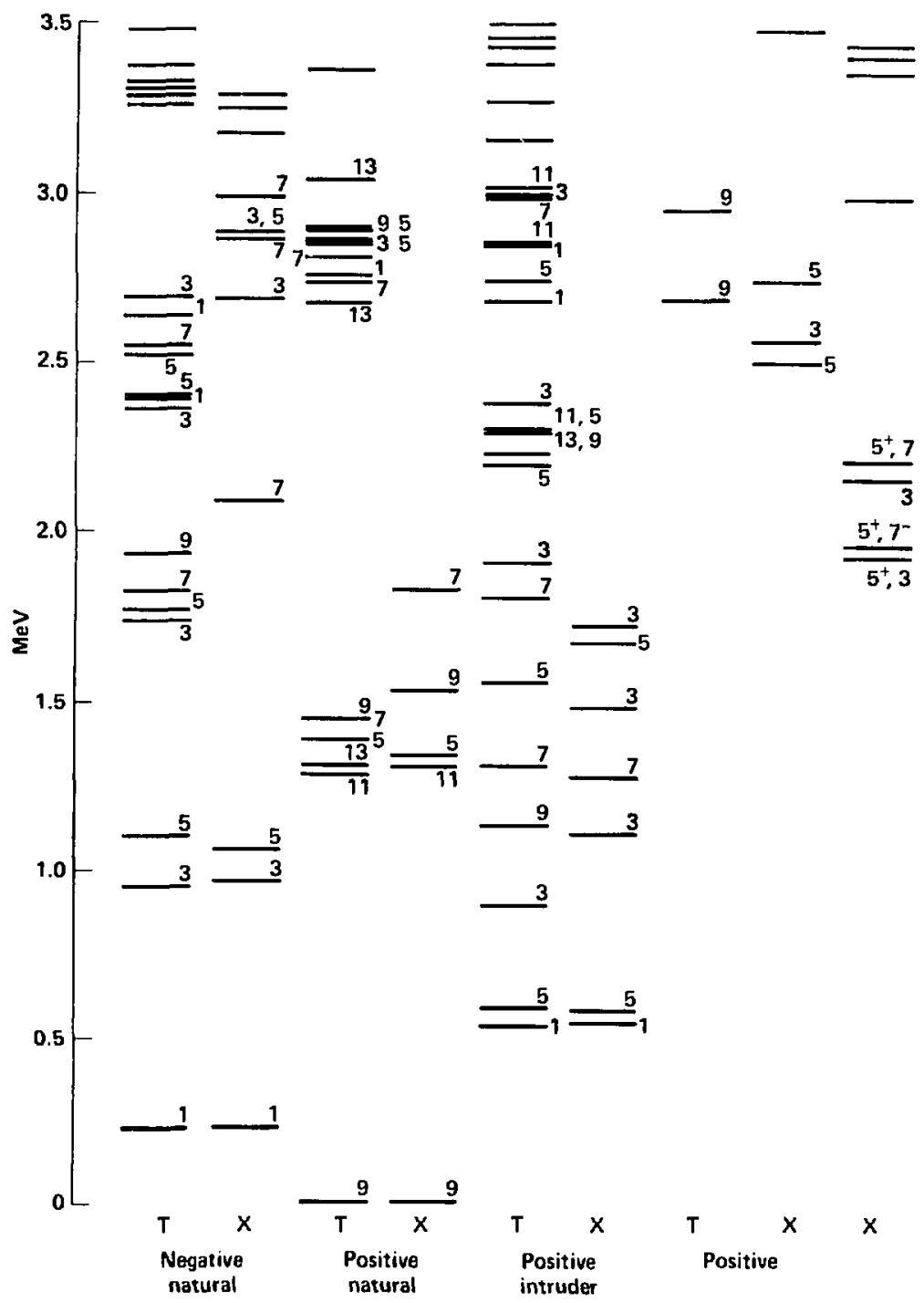

Fig. VII.I. Comparison of experimental and theoretical level energies for ${ }^{83}$ Se. Each column is labeled theory $(T)$ or experiment $(X)$. The first four pairs of columns are the natural negative parity levels, the natural positive parity levels, the positive parity intruder levels (I), and the positive parity mixed levels. The last column has the experlmental levels of unknown parity. The spins of the levels are given as 2J. All the negative parity levels are in the first two columns, the remainder are positive or unknown parity. 


\section{Intruder Levels}

Experimentally, the intruder levels do not have any multiplet grouping in energy. Theoretically, all of the intruder levels are of mixed composition, usually including some small natural component. By mixed composition I mean that the level has no component with a squared expansion coefficient which was greater than 0.4 , with most being less than 0.3 . This mixing suggests that the multiplet structure would be substantially shifted as observed in experiment. Many of the calculated levels, especially above $2 \mathrm{MeV}$, were not found in the experiment, although this may be due to the levels not being populated in $\beta$-decay.

The unified model gives much better prediction on number and energy of $1 / 2+$ states than does the weak coupling model. The weak coupling limit predicts the following eleven $1 / 2^{+}$states below an excitation energy of $3 \mathrm{MeV}:\left[(0,0) 1 / 2^{+}\right],\left[(2, U) 1 / 2^{+}\right],\left[(3,0) 1 / 2^{+}\right],\left[(1,2) 3 / 2^{+}\right],\left[(2,2) 3 / 2^{+}\right]$, $\left[(3,2) 3 / 2^{+}\right],\left[(1,2) 5 / 2^{+}\right],\left[(2,2) 5 / 2^{+}\right],\left[(3,2) 5 / 2^{+}\right],\left[(3,3) 5 / 2^{+}\right],\left[(2,4) 9 / 2^{+}\right]$where the level energy is determinet in the weat coupling way as

$E_{\mathrm{x}}=n \hbar \omega+E($ single particle $)$

and $n$ is the number of phonons of energy $h \omega$ and $E$ is the excitation energy of $t \quad$ vest state of spin $\mathrm{j}$. The states based on the $7 / 2^{+}$single particle were ignorrd since ro spectroscopic factor was found for the $1 \mathrm{~g} 7 / 2$ in the $(\overrightarrow{\mathrm{d}}, \mathrm{p})$ experiment. The unified model predicts only $4 \mathrm{i} / 2^{+}$levels below $3 \mathrm{MeV}$, three of which lie between 2.5 and $3 \mathrm{MeV}$. Experimentally, there ate three $1 / 2^{+}$levels identified below $4 \mathrm{MeV}$, with four more levels which could be $1 / 2^{+}$, but with $3 / 2^{+}$preferred and one possible assignment of $1 / 2^{+}$. Figure VII. 2 shows the $1 / 2^{+}$levels found by the weak coupling model, experiment and the unified model. The unified model predicts the gap from 0.6 to 2.3 $\mathrm{MeV}$, with no $1 / 2^{+}$levels, while the weak coupling limit does not.

\section{Spectroscopic Factor for $(\vec{d}, p)$ Reaction}

The single particle transfer reaction serves as a good test of the model states. For ${ }^{83} \mathrm{Se}$ only the ${ }^{{ }^{2} 2} \mathrm{Se}\left(\overline{\mathrm{d}}_{\mathrm{p}} \mathrm{p}{ }^{\mathrm{B} 3}\right.$ Se reaction has been studied. This reaction observes the spectroscopic $f=$ or of the intruder single particle only. In Fig. VII.3 the theoretical and experimental spectros pic factors are compared. The theoretical spectroscopic factors are generally a factor of 2 lower tnan experiment. This difference is consistent with that found in the indium nuclei (HEY78, HEY80, GLA7?). A further test of the model is a comparison of the theoretical and experimental values for the summed spectroscopic factor,

$s_{r_{1}}=\sum_{1} s_{r_{\prime \prime}}$

the mean energy $E_{\text {, }}$

$E_{1}=\frac{\sum E_{1} S_{P_{l}}}{S_{P_{1}}} ;$

and the width of the spectroscopic factor distribution,

$w_{i}^{2}=\frac{\sum\left(E_{l}-E_{l}\right)^{2} S_{f_{H}}}{S_{f_{l}}}$. 


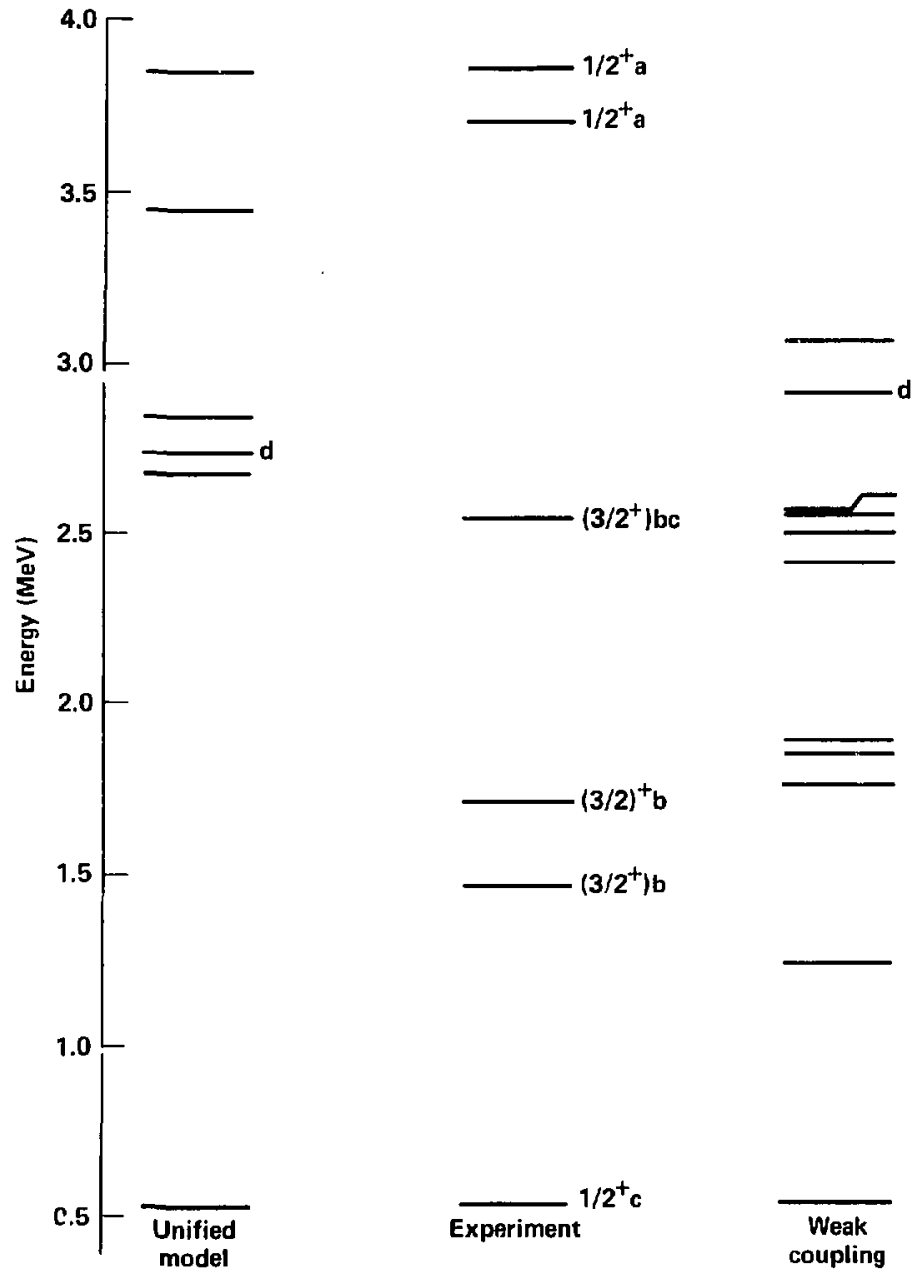

Fig. VII.2. Comparison of the $1 / 2^{+}$states found by the unified model, experiment, and the weak coupling limit. Notes: a) LIN65, b) this work, c) MON78, d) natural level.

In Table VII.1, I present these values for $j=3 / 2^{+}$and $j=5 / 2^{+}$. Much better agreement is obtained for the averaged values than for the individual levels.

There are two interesting discrepancies between theory and experiment. The $5 / 2^{+} 2.18 \mathrm{MeV}$ theoretical level has a spectroscopic factor of 2.77 which is much higher than any of the experimental levels. This would seem to indicate that the $5 / 2^{+}$spectroscopic factor does not spread 


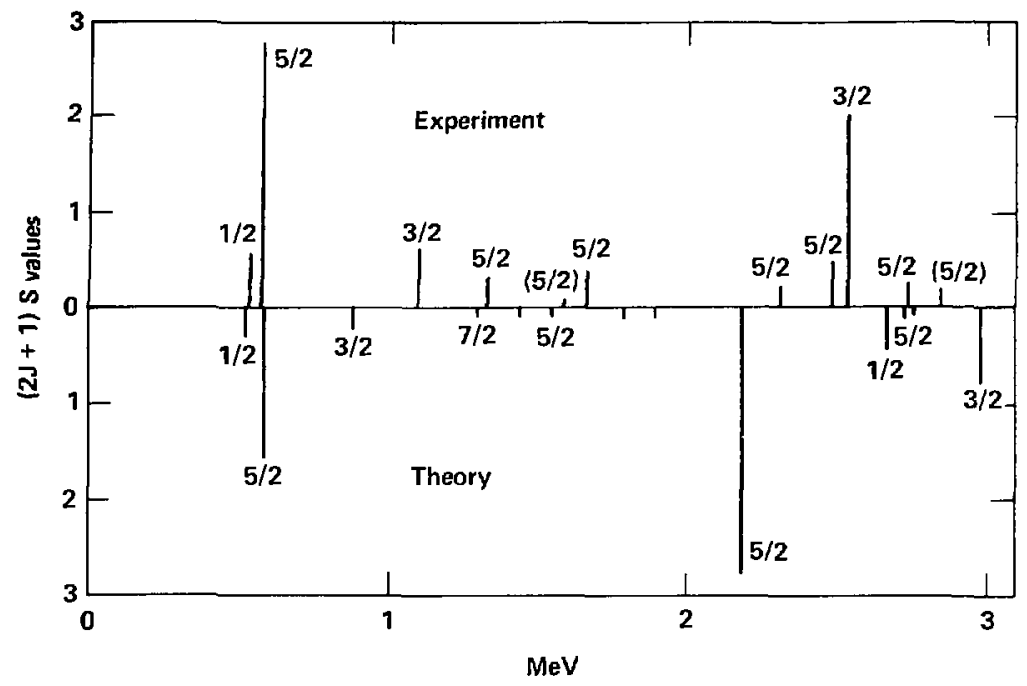

Fig. VII.3. Comparison of spectroscopic factor for theory and experiment in the ${ }^{82} \mathrm{Se}(\overrightarrow{\mathrm{d}}, \mathrm{p}){ }^{83}$ Se reaction. All stites shown are positive parity.

Table VIl.1. Comparison of spectroscopic factor distribution for theory and experiment for the ${ }^{82} \mathrm{Se}(\overrightarrow{\mathrm{d}}, \mathrm{p})^{83}$ Se reartion. Experimental values are from determined from Ref. MON78.

\begin{tabular}{|c|c|c|c|c|}
\hline & \multicolumn{4}{|c|}{ Levels of Spin and Parity } \\
\hline & \multicolumn{2}{|c|}{$3 / 2$} & \multicolumn{2}{|c|}{$5 / 2$} \\
\hline & Theory & Enperiment & Theory & Experiment \\
\hline$s_{i r}$ & 1.4 .5 & 2.58 & 4.28 & 5.32 \\
\hline$\Gamma_{1}$ & 2.84 & 2.20 & 1.66 & 1.50 \\
\hline$w_{i}$ & 0.92 & 0.60 & 0.77 & 1.08 \\
\hline cutoff & 4.2 & 3.7 & - & - \\
\hline
\end{tabular}

enough in the calculation. On the other hand, there is no theoretical level with the spectroscopic factor held by the experimental $3 / 2+2454-\mathrm{keV}$ level, which suggests the opposite difficulty for the $3 / 2^{4}$ levels.

\section{Gamma-Ray Branching Ratios}

\section{Natural Levels}

I discuss the gamma-ray branching ratios for the low lying levels and the $7 / 2$ levels as determined by my experiment. First, 1 will discuss the $9 / 2^{+}$level and its associated multiplet; next the natural $7 / 2$ levels; and last the intruder levels. 
Figure VII.1 shows the good agreement obtained between theory and experiment for the energy of the four observed members of the $\left[(1,2) 9 / 2^{+}\right]$multiplet found experimentally. I compare the theoreticai and experimental gamma-ray branching ratios from these levels in Fig. VIl.4. The gamma-ray branch to the ground state dominates for each of these levels. The $\Delta l=1$ intramultiplet transitions all show good agreement between theory and experiment. The $13 / 2^{+}$ member of this multiplet is not observed, as a two gamma-ray cascade is required to populate such a high spin state from a $7 / 22^{-}$state populated via $\beta^{-}$decay.

Most of the large discrepancies occur where the experimental branching ratios are very small and uncertain, hence the discrepancies are not significant. The theoretical branching ratio for the $557-\mathrm{keV}$ transition from the $7 / 2^{+} 1822 \mathrm{keV}$ level to the $7 / 2+1265-\mathrm{keV}$ level is 60 times too large. This discrepancy will be discussed when I discuss the $7 / 2^{+}$with the intruder levels.

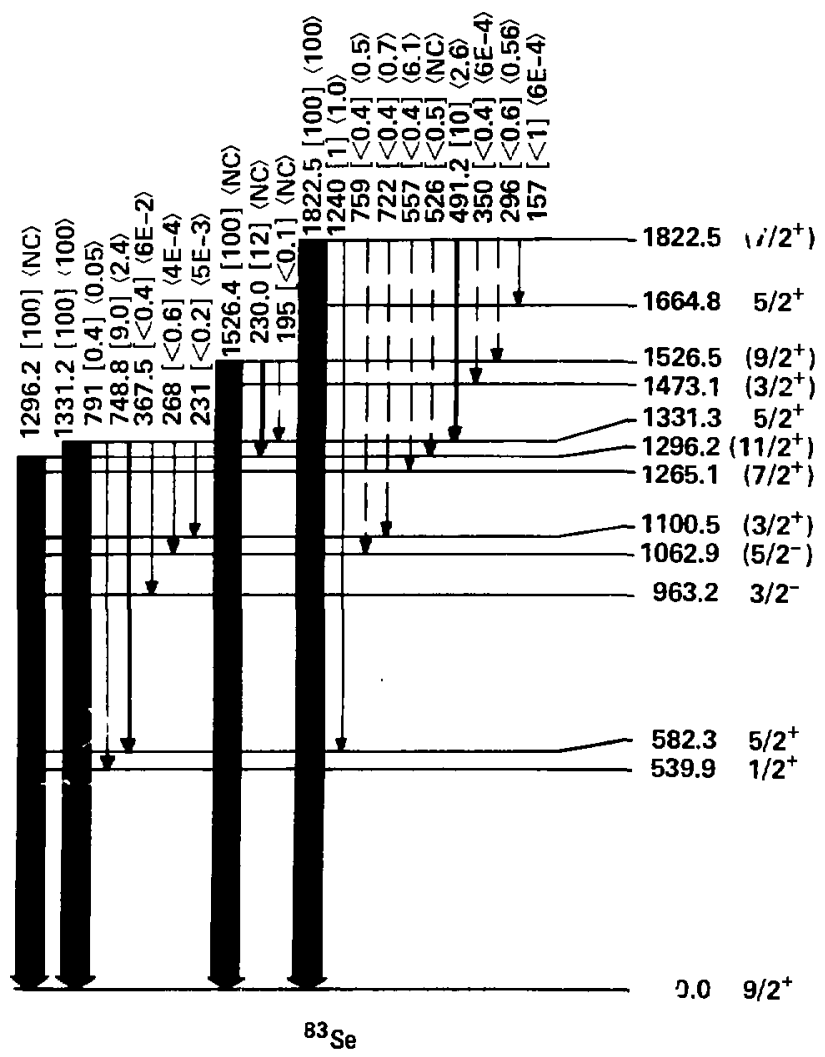

Fig. VIl.4. Gamma transition branching ratios for theory and experiment for the $\left[(1,2) 9 / 2^{+}\right]$ multiplet in ${ }^{83} \mathrm{Se}$. Branching ratios are normalized to 100 for the most intense experimental transition. The branching ratius are given in square brackets fexperimentall and in angle brackets (theory), (NC) means that the theoretical transition was not calculated. 
From the gamma-ray branching ratios, the $5 / 2^{+} 1331-\mathrm{keV}$ level is a member of the $\left[(1,2) 9 / 2^{+}\right]$ multiplet. Theoretically the branch to the $5 / 2^{+} 582 \cdot \mathrm{keV}$ intruder level is a factor of 4 weaker than experiment. The theoretical $(2 J+1) S$ value is 0.00 , while the experimental value is 0.33 . The major

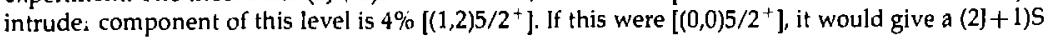
value of 0.24 , but it is the wrong component.

\section{The $7 / 2 \cdots$ States}

These levels are interesting because they can have no seniority one component, except for a vanishingly small $1 \mathrm{f} 7 / 2$ hole component, and have not been previously identified in $N=49$ nuclei. In Fig. VII.5, I show these levels and their branching ratios for $M 1 / E 2$ transitions. (All these levels have intense $E 1$ transitions to the ground state, but the theory makes poor predictions for $E 1$ transitions.)

The configuration of these $7 / 2^{-}$states can be a) $\left[(2,4) 1 / 2^{-}\right]$b) $\left[(1,2) 3 / 2^{-}\right]$or $\left.c\right)[(1,2) 5 / 2]$ for levels at 2077, 2858, and $2981 \mathrm{kcV}$. The $3 / 2 \quad 963-$ and $5 / 2 \quad 1063-\mathrm{keV}$ levels can have configurations $f(0,0) 3 / 2] 3 / 2$ and $\left[(1,2)_{1 / 2}\right] 3 / 2$; and $\left[(0,0) 5 / 2^{-}\right] 5 / 2$ and $\left.[11,2) 1 / 2 "\right] 5 / 2$ respectively. The relative $B(E 2)$ for transitions between these configurations are:

1) for $7 / 2 \bowtie 3 / 2$

$[(1,2) 3 / 2] 7 / 2 *[(0,0) 3 / 2] 3 / 2-1.0$

$[(2,4) 1 / 2] 7 / 2 \cdot\left[(1,2) 1 / 2^{-}\right] 3 / 2^{-} \quad 1.7$

2) for $7 / 2 \bullet 5 / 2$

$[(1,2) 5 / 2] 7 / 2 \otimes[(0,0) 5 / 2-15 / 2-1.0$

$\left[(2,4) 1 / 2 \quad 7 / 2 \otimes\left[(1,2) 1 / 2^{-}\right] 5 / 2^{-} \quad 0.2\right.$

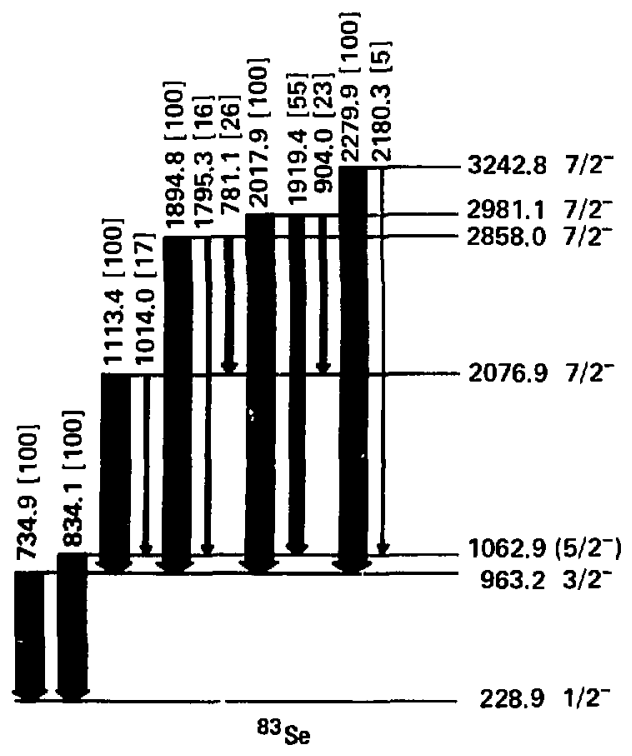

Fig. VII.5. Gamma transition branching ratios for theory and experiment for the $7 / 2^{-}$ levels. Other information is the same as for Fìg. v1l.4. 
l assume that the $7 / 2$ to $5 / 2$ transition is predominately an $E 2$ transition. The $M 1$ is a single particle transition, while the $E 2$ is a collective transition. This suggests that the $7 / 2^{-}$levels are predominately of configurations $a$ and $b$, for if configuration $c$ is a major component of the $7 / 2^{-}$ levels, the transition to the $5 / 2$ state should be enhanced. Based on the transition branching ratios, only the $2981-\mathrm{keV}$ level could be of configuration c. Any $M 1$ contribution to the transition to the $5 / 2$ state only emphasizes the $1 / 2^{-}$multiplet component, as this component allows no $M 1$ transition.

The calculated coefficients for the components of the $7 / 2^{-}$levels are given in Table VIl.2. They show reasonable agreement with the above evaluation, with the $1 / 2^{-}$and $3 / 2^{-}$based components dominating.

\section{The Intruder Levels}

The intruder levels are all positive parity and can be expected to mix with each other. The major components are $2 \mathrm{~d} 5 / 2,3 \mathrm{~s} 1 / 2$, and $2 \mathrm{~d} 3 / 2$, with the $1 \mathrm{~g} 7 / 2$ being small. Further the natural $\lg 9 / 2$ configurations will mix with the iritruder configurations to varying degrees.

Our measured value for the half-life of the $5 / 2^{+} 582-\mathrm{keV}$ level, $3.1 \mathrm{~ns}$, shows that the transilion to the $9 / 2$ ' ground state is hindered by a factor of 8.1 relative to the single particle estimate (NDS81). The unified model calculations show a hindrance factor of 1.6. Both of these hindrance values are significantly different from the core $E 2$ transitions which have enhancement values of 7 for the ${ }^{\mathrm{Hit}} \mathrm{Se}$ core, and 17 for the ${ }^{82} \mathrm{Se}$ core. This is consistent with the cores for the intruder and natural utates being substantially different which precludes the transition being a simple single particle transition.

The transition from the $5 / 2_{i}^{+}$level to the $1 / 2_{1}^{+}$level, which might be expected from similar highly enhaneed transitions in the indium nuclei, has as a branching ratio less than 0.3 . Including the salue for the internal conversion coefficient, 19 (ROS78), this limit corresponds to a maximum 10-fold enhancement of the transition. This enhancement is comparable to those observed in the indium nuclei. The model calculations obtained a branching ratio of $4 \mathrm{E}-3$, an enhancement of 12 , which is consistent with the experimental results.

The $1 / 2 i, 5 / 2 ;, 3 / 2 i, 7 / 2+$, and $5 / 2+$ are the low-lying intruder levels. The energy of these levels dee' nut display the particle plus vibrational core multiplet structure, such as was observed with the $1 \mathrm{~g} 9 / 2$ hole plus $\mathrm{N}=50$ vibrational core, nor do the gamma-ray transitions fit that description. Figure VII.6 show's the experimental energy levels and compares experimental and theoretical gamma-ray branching ratios. These levels have been identifieu as having i uder components in the $(\vec{f}, p)$ reaction (MON78), except for the $7 / 2^{\circ}$, and the gamma-ray branching ratios confirm this identity. The multiplet structure is destroyed by the strength of the particle-vibration coupling and the presence of several single particle orbitals at low energy. The analogous situation occurs in the indium nuclei.

Experimentally the largest branch is always to the $5 / 2^{+} 582-\mathrm{keV}$ level, with a snialler branch to the $1 / 2{ }^{\prime} 54^{r} . V$ level, where allowed. Branches to the natural states are alwavs smaller as expected. Branches between the $3 / 2+, 7 / 2$, and $5 / 2^{+} 1665-\mathrm{keV}$ levels are also very small.

Table VII.2. Selected expansion coefficients for the first three $7 / 2$ states and the $3 / 2_{1}^{-}$and $5 / 2$, states. These can be ised for estimating the gamma-ray branching ratios from the $7 / 2$ states to the $5 / 2$, and the $3 / 2$, states.

\begin{tabular}{lcccccc}
\hline$J^{2}$ & \multicolumn{5}{c}{ Expansion coefficient for } \\
\cline { 2 - 6 } & {$[(2,4) 1 / 2]$} & {$[(1,2) 1 / 2]$} & {$[(1,2) 3 / 2]$} & $[1], 2) 5 / 2]$ & {$[(0,0) 3 / 2]$} & {$[(0,015 / 2]$} \\
\hline $7 / 2$, & 0.579 & - & 0.672 & 0.158 & - & - \\
$7 / 2$, & 0.074 & - & 0.233 & 0.768 & - & - \\
$7 / 2$, & 0.597 & - & 0.305 & 0.119 & - & - \\
$3 / 2$, & - & 0.558 & 0.278 & 0.162 & 0.688 & 0.619 \\
$5 / 2$, & - & 0.618 & 0.152 & 0.264 & - & 0.619 \\
\hline
\end{tabular}




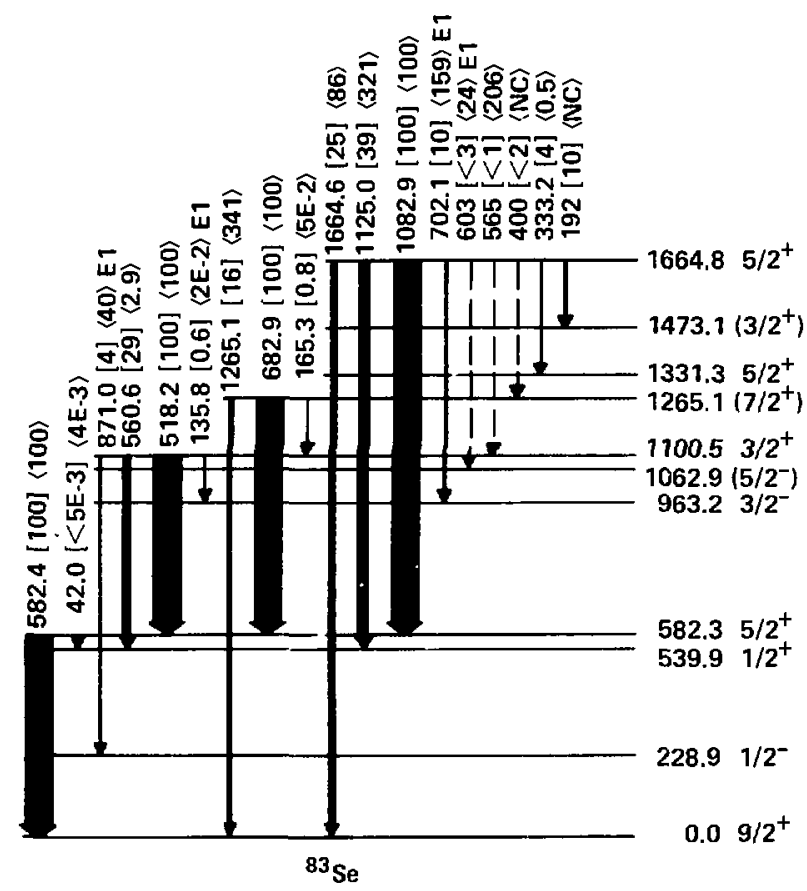

Fig. VII.6. Gamma transition branching ratios for theory and experiment for the intruderone-phonon levels. Other information is the same as for Fig. VII.4.

Theoretically, the major branches from the $3 / 2^{+}$and $5 / 2^{\prime} 1665-\mathrm{keV}$ level are to the intruder levels. The specific branching to the intruder levels is not well reproduced. Without experimental $M 1 / L 2$ mixing ratios and sxperimental reduced transition probabilities, it is not possible to explain these differences.

The two (3/2) ' levels at 1472 and $1710 \mathrm{keV}$ (Fig. VII.7) feed the lower intruder levels strongly. Their theoretical energies were off by $300 \mathrm{keV}$. The spectroscopic factors were very low both in theory and experiment. The experimental branching ratios from the $1710-\mathrm{keV}$ level are well reproduced by the theory. The experimental branching ratios from the $1472-\mathrm{keV}$ level appear poorly reproduced.

The unified model correctly predicts the main features of the low-lying level structure in ${ }^{83} \mathrm{Se}$. The intruder band structure is confirmed. The deficiencies in the intruder band branching ratios are probably cancellation effects (HEY82b). The misplaced gamma-ray transition intensity from the intruder levels and the missing spectroscopic factor from the $5 / 2^{+}$natural level may be caused by incorrect mixing of the intruder and natural levels. Also possible are the missing contributions from 2-particle 3-hole configurations. Lin (LIN65) has measured a substantial component of $n(d 5 / 2)_{10}^{2 *}$ in the ${ }^{42}$ Se ground state, which suggests the 2-particle 3-hole configuration may be present in the low-lying levels of ${ }^{83} \mathrm{Se}$. These configurations are not treated in the unified model. 


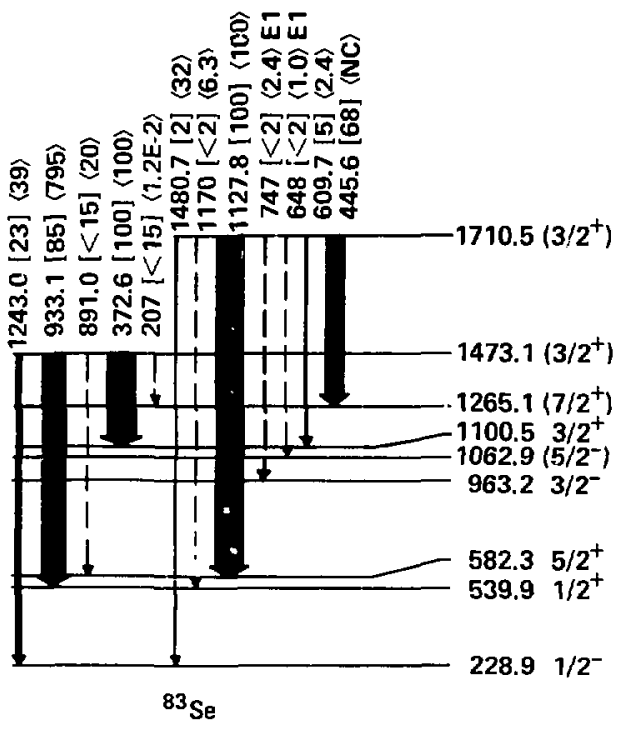

Fig. V1l.7. Gamma transition branching ratios for theory and experiment for the $3 / 2^{+}$ intruder-two-phonon multiplet. Other information is the same as for Fig. VII.4.

\section{Theoretical Systematics}

In the earlier section on systematics of the $N=49$ isotones and $Z=49$ (indium) isotopes, 1 pointed out that while the natural levels remain at nearly constant energy in both systems, the energy of the intruder levels varies substantially. In the indium nuclei the intruder level energies are lowly changing (Fig. V.1) but in the $\mathrm{N}=49$ isotones the level energies are rapidly increasing in energy from $Z=32$ germanium to $Z=40$ zirconium (Fig. V.3). I noted that this behavior is similar to the hehavior of the energy of the $2{ }^{+}$level in the respective core nuclei. I will now look at the unified model parameters used to reproduce the systematic behavior in these two groups of nuclei.

In Tables VII.3 and VII.4, 1 present the unified model parameters used to reproduce the 49 nucleon systematics, $Z=49$ and $N=49$ respectively. The parameter $h u_{2}$ is taken from the enere.' of the $2^{\prime}$ excited state of the appropriate nucleus as previously discussed. The dimensionless coupling coefficient is constant for iidium nuclei, and for the $N=50$ core in the $N=49$ case. However, for the $N=48$ core it varies dramatically, from 6 for selenium to 3.5 for strontium. The energy required to create the particle hole pair also changes much more rapidly in the 49 isotones than in the indium nuclei. The single particle energies do not change at all in indium, and only slightly in the $\mathrm{N}=49$ isotones.

The changes in the collective parameters of the unified model reproduce the systematic behavior of the intruder levels in the $\mathrm{N}=49$ nuclei (Fig. VII.8). Further, the differences in behavior among the lowest $1 / 2^{\prime}, 3 / 2^{\prime}$, and $5 / 2{ }^{\circ}$ intruder states are also correctly reproduced, without recourse to the single particle energies. I note that the $3 / 2$ + level energy changes most, but the corresponding single particle energy not at all. 
The collective nature of the $Z$ or $N=48$ nucleus appears to be the dominant factor in the appearance of the intruaer states. In the indium case, far from shell closure, the intruder levels are at low energy and vary slowly with neutron number. The minimum is at mid neutron shell 117 In. In the $\mathrm{N}=49$ case, the intruder levels are at low energy in ${ }^{81} \mathrm{Ge}$ and ${ }^{83} \mathrm{Se}$, at mid proton shell. These are much closer to shell closure than in the indium case ( 3 nucleon pairs vs. 7 nucleon pairs) and the intruder levels rise rapidly to the proton shell closure at $Z=40$.

Table VII.3. The parameters used for the $N=49$ nuclei to reproduce the systematic behavior of the intruder and natural levels. The parameters are the quadrupole phonon energy, $h \omega_{2}$, the dimensionless coupling coefficient, $E_{2}$, the energy required to create a particle hole pair, $\Delta E_{p h}$, and the single particle energies, $\epsilon$. All parameters are in $\mathrm{MeV}$, except for $E_{2}$.

\begin{tabular}{|c|c|c|c|c|}
\hline & ${ }_{37}^{81} \mathrm{Ge}_{44}$ & ${ }_{34}^{43} \mathrm{Se}_{49}$ & ${ }_{16}^{85} \mathbf{K}_{\mathbf{r}_{49}}$ & ${ }_{38}^{87} \mathrm{Sr}_{49}$ \\
\hline$h \omega_{2}(\mathrm{~N}=50)^{n}$ & 1.400 & 1.400 & 1.565 & 1.836 \\
\hline$h_{\omega_{2}}(N=48)^{4}$ & 0.655 & 0.655 & 0.662 & 1.077 \\
\hline$E_{2}(N-50)$ & 2.0 & $2.0^{b}$ & 2.0 & 2.0 \\
\hline$C_{2}(N \cdot 48)$ & 5.75 & 6.00 & 4.50 & 3.50 \\
\hline${ }^{\prime} x^{4 / 2}$ & $\sigma^{r}$ & 0 & 0 & 0 \\
\hline 'pizz & 1.30 & 0.70 & 0.80 & 1.00 \\
\hline tp.3:2 & 2.30 & 0.70 & 1.70 & 1.50 \\
\hline $1 / 4 / 2$ & 2.55 & 1.65 & 1.90 & 2.00 \\
\hline$\Delta \mathbf{E}_{\mathbf{F h}}$ & 2.0 & 2.0 & 2.50 & 2.80 \\
\hline$\sqrt[4]{4 i 2}$ & 0 & 0 & $\mathbf{0}$ & 0 \\
\hline 1362 & 1.40 & 1.50 & 1.60 & 1.80 \\
\hline $1203 / 2$ & 2.60 & 2.60 & 2.60 & 2.60 \\
\hline$t_{B} \div 2$ & 2.80 & 2.80 & 2.80 & 2.80 \\
\hline 'tht1/2 & 2.60 & 2.60 & 2.60 & 2.60 \\
\hline
\end{tabular}

"Experimental value.

$\mathrm{A}$ value of 1.0 was used in the calculations for ${ }^{\mathrm{B}} \mathrm{Se}$.

' Zcro values without decimal point are zero by definition.

Table VII.4. The parameters used fo: the $Z=49$ nuclei to reproduce the systematic behavior of the intruder and natural levels. The parameters are the quadrupole phonon energy, $h \omega_{2}$, the dimensionless coupling coefficient, $E_{2}$, the energy required to create a particle hole pair, $\Delta E_{b,}$, and the single particle energies, $t$. All parameters are in $\mathrm{MeV}$, except for $E_{2}$.

\begin{tabular}{|c|c|c|c|c|}
\hline & $\mathrm{JW}_{40}^{115} \mathrm{n}_{\mathrm{mb}}$ & ${ }_{49}^{113} \mathrm{In}_{68}$ & ${ }_{40}^{119} \ln _{70}$ & ${ }_{49}^{121} \mathrm{In}_{72}$ \\
\hline$h \omega_{2} \mid Z=50 y^{*}$ & 1.293 & 1.3 & 1.17 & 1.14 \\
\hline$f\left(w_{2}(Z=48)^{4}\right.$ & 0.538 & 0.54 & 0.487 & 0.506 \\
\hline$l_{3}(Z-50)$ & 2.4 & 2.4 & 2.4 & 2.4 \\
\hline$f_{2}(2-48)$ & 6.1 & 7.6 & 7.6 & 7.6 \\
\hline [ky/ & $0^{b}$ & 0 & 0 & 0 \\
\hline 'plis & 0.60 & 0.60 & 0.60 & 0.60 \\
\hline$t p 3 / 2$ & 1.30 & 1.30 & 1.20 & 1.20 \\
\hline$t_{15 / 2}$ & 2.00 & 2.00 & 1.90 & 1.90 \\
\hline$\Delta \mathbf{E}_{\rho h}$ & 2.4 & 2.34 & 2.5 & 2.5 \\
\hline${ }^{4} \mathrm{~K}^{7 / 2}$ & 0 & 0 & 0 & $\mathbf{0}$ \\
\hline $\mathrm{cds}_{\mathrm{d} / \mathrm{s}}$ & 0.50 & 0.50 & 1.0 & 1.0 \\
\hline 'titilit? & 2.10 & 2.10 & 2.10 & 2.10 \\
\hline 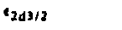 & 2.60 & 2.60 & 2.60 & 2.60 \\
\hline$t 3.1 / 2$ & 2.95 & 2.95 & 2.95 & 2.95 \\
\hline
\end{tabular}

- Experimental.

'Zero values without derimal point are zero by definition. 


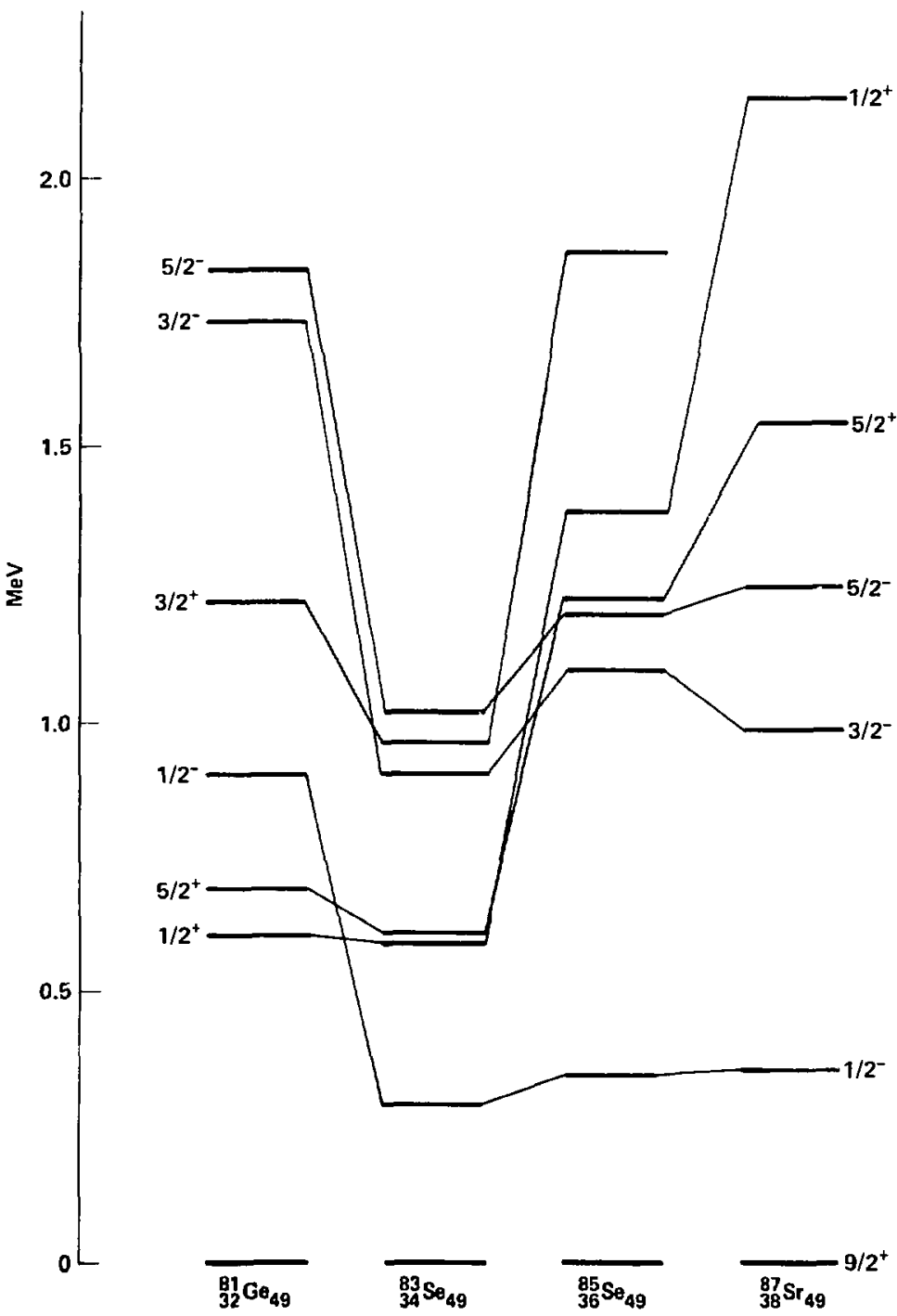

Fig. VII.B. Theoretical $N=49$ level systematics showing the lowest natural levels of spin and parity $9 / 2,1 / 2,3 / 2$, and $5 / 2^{-}$, and the lowest intruder states of spin and parity $1 / 2^{\prime}, 3 / 2^{\prime}$ and $5 / 2^{\prime}$. 


\section{Future Experiments}

Several more experiments are needed to further illuminate shape coexistence in the $N=50$ region. In these experiments particular attention should be given to determining level lifetimes and $M 1 / E 2$ ratios as required for a more detailed comparison with the theoretical predictions. Also, a specific search needs to be made for the missing $1 / 2^{+}, 7 / 2^{+}$, and $11 / 2^{-}$spectroscopic factor. Where gamma rays are observed angular correlation/distribution of the gamma rays must be observed. Only with this information will it be possible to check the theoretical gamma-ray transition intensities.

The following studies would be useful in studying the $N=49$ isotones:

- ${ }^{82} \mathrm{Se}(\mathrm{n}, \gamma)^{\mathrm{H3}} \mathrm{Se}$ - This experiment would locate the low spin states. particularly to confirm the few $1 / 2^{+}$states which have been found and are predicted by the unified model. The gamma rays should also cascade through the $1 / 2^{+}$states which have no observable $[(u, 0) 0,1 / 2\}$ component.

- ${ }^{82} \mathrm{Se}(\mathrm{d}, \mathrm{p} \gamma)^{\mathrm{H} 3 \mathrm{Se}}$ - to study the $3 / 2^{+}$and $5 / 2^{+}$states which are predominately populated in this experiment. This experiment would give a clearer picture of the intruder structure as the intruder component is directly populated, rather than the natural component as in the $\beta$ decay experiment I performed. Also possible are the $(\mathrm{t}, \mathrm{d})$ and $(\mathrm{t}, \mathrm{d} \gamma)$ experiments. These later experiments should locate the $7 / 2^{+}$spectroscopir. factor, if it is present.

- ${ }^{\mathrm{H}} \mathrm{Kr}(\mathrm{n}, \boldsymbol{a \gamma})^{\mathrm{B} 7} \mathrm{Se}$ - to study the more complex states which may not be excited by the neutron transfer reactions mentioned above. This reaction will be difficult as the reaction has $\mathrm{Q}=-2.7 \mathrm{MeV}$ and krypton is a difficult target to use.

- ${ }^{{ }^{4} 4} \mathrm{Kr}(\overrightarrow{\mathrm{d}}, \mathrm{p}),{ }^{\mathrm{k}} \mathrm{Kr}(\overrightarrow{\mathrm{p}}, \mathrm{d}),{ }^{\mathrm{k}} \mathrm{Kr}(\mathrm{d}, \mathrm{p} \gamma)$, and ${ }^{\mathrm{k} 6} \mathrm{Kr}(\mathrm{p}, \mathrm{d} \gamma){ }^{\mathrm{H}} \mathrm{Kr}$ - reactions to study the development of the collective intruder structure in transition. Some of these experiments have been done but incompletely; neither polarized projectiles nor in-beam gamma rays have been used.

- Identification of other $7 / 2$ levels in the $N=49$ nuclei would shed light on the extent to which vibrational structure of the natural levels is affected by changing collectivity in these nuclei.

For the $\mathrm{N}=51$ nuclei, which have a single particle outside the closed shell, the conjugate structure to that found in the $N=49$ nuclei is expected, with the particle and hole states being the natural and intruder states respectively. To the extent that the shell closure at $Z=40$ affects the intruder structure as it does for the $\mathrm{N}=49$ isotones, the most likely candidates for study are ${ }^{95} \mathrm{Ru}$ and ${ }^{47} \mathrm{Pd}$.

The following studies would be useful in studying the $N=51$ isotones:

Studies of levels " ${ }^{\prime 5} \mathrm{Ru}$ have been studied using $\beta^{+}$decay (WEI75), (p,d) (BAL71), and ( $\left.\alpha, n \gamma\right)$ (L.ED71). Only the $\beta$ ' decay study is relatively complete. The $(\alpha, n \gamma)$ reaction revealed only four levels.

- ${ }^{40} \mathrm{Ru}(\overrightarrow{\mathrm{p}}, \mathrm{d})^{45} \mathrm{Ru}$ and ${ }^{46} \mathrm{Ru}(\mathrm{F}, \mathrm{d} \gamma)^{95} \mathrm{Ru}$ to find the intruder states and their bands, with the polarized protons to determine the correct spins.

- ${ }^{4-1} \mathrm{Mo}(\alpha, 3 \mathrm{n} \gamma)^{45} \mathrm{Ru}$ and a more careful study with the ${ }^{92} \mathrm{Mo}(\alpha, \mathrm{n} \gamma)^{4.5} \mathrm{Ru}$ reaction might reveal more of the band structure.

- ${ }^{4 h} R u(n, 2 n)^{47} R u,{ }^{4} R u(n, 2 n \gamma){ }^{45} R u$ are also possible studies.

- ${ }^{46} \mathrm{Ru}\left({ }^{3} \mathrm{He}, 2 \mathrm{n} \gamma\right)^{47} \mathrm{rd}$ and ${ }^{1 / 6} \mathrm{Ru}(\alpha, 3 \mathrm{n} \gamma)^{47} \mathrm{Pd}$ reactions would give spins and parities and transition intensitjes. The ${ }^{9 h} \mathrm{Ru}\left({ }^{3} \mathrm{He}, 2 \mathrm{n} \gamma\right)$ was done by Fettweis et al. (FET82). 


\section{Conclusions}

In this thesis work 1 have developed AUTOBATCH, which utilized computer controlled automation to chemically prepare large numbers of replicate samples of short lived fission products for gamma-ray spectroscopy. With AUTOBATCH the gamma rays following the $\beta^{-}$decay of ${ }_{33}^{83} \mathrm{As}_{50}$ were studied to determine:

- the ground state spin and parity of ${ }^{83} \mathrm{As}$ to be $5 / 2^{-}$;

- the absolute intensity of the $\beta^{-}$branch from ${ }^{83} \mathrm{As}$ to ${ }^{83} \mathrm{Se}^{\mathrm{m}}$ to be $0.3 \%$;

- the absolute intensity of the ground state $\beta^{-}$branch from ${ }^{83} \mathrm{Se}^{\mathrm{m}}$ to ${ }^{83} \mathrm{Br}$ to be $38 \%$ through a better normalization;

- the half-life of the $5 / 2 i_{1}^{+}$level to be $3.2 \mathrm{~ns}$;

- the structure of ${ }_{34}^{83} \mathrm{Se}_{49}$.

The results are used to show that the intruder vibrational structure which had been previously observed in the odd mass ${ }_{4 y}$ In isotopes could be observed in the $N=49$ isotones. The intruder vibrational structure is most strongly developed, not at core mid-shell, ${ }_{40}^{89} \mathrm{Zr}_{49}$, but rather at core mid-sub-shell, ${ }_{34}{ }^{3 / 3} e_{19}$. This difference is qualitatively understood to be due to the blocking of collectivity by the subshell closure at $Z=40$ which prevents the intruder vibrational structure from occurring in ${ }^{87} \mathrm{Sr}$ and ${ }^{\mathrm{k9}} \mathrm{Zr}$. 


\section{Appendix 1. AUTOBATCH Details}

\section{Transport Capsule}

The transport capsule, called a rabbit, was a hollow polyethylene cylinder $6.2 \mathrm{~cm}$ long by $2.8 \mathrm{~cm}$ diameter, with a mass of $21 \mathrm{~g}$ when loaded. Both ends had thin sections which were easily penetrated by the extraction needle. The rabbit caused two problems which were solved ty modifying the handling equipment and procedures.

The rabbit traveled at $50 \mathrm{~m} / \mathrm{s}$, abrading polyethylene fuzz from the rabbit. This fuzz interfered with the photo sensors. Daily cleaning eliminated this problem.

The rabbit was sufficiently elastic that it bounced when it hit the receiver. This bouncing made it difficult to determine when the rabbit was stopped on the bottom of the receiver. The new receiver reduced both of these problems to acceptab!e levels.

\section{Loader/Launcher}

The original loader was a manually loaded single shot loader implemented with a "quick disconnect" fitting. Loading was so physically difficult that rabbits could only be loaded one a minute. Loading rabbits into the launcher required one person full time.

The new launcher was a modified NURE launcher. The rabbit was loaded through a breech operated by a pneumatic cylinder. A supply of rabbits was held in a gravity feed magazine above the breech. The magazine could be loaded in two minutes, without interrupting sample processing. A photosensur activated an audible alarm when there were three rabbits left in the magazine. The only difficulty with this launcher occurred when the magazine was allowed to run to empty. The last rabbit would not drop into the breech, causing the loader to jam. The major advantages were elimination of one worker and reduction of the loading time from sixty to five seconds. Recycle imes down to $15 \mathrm{~s}$ were not limited by the launcher.

\section{Receiver}

\section{Original Receiver}

The old receiver used a three position moving block assembly to manipulate the rabbit. The rabbit was received in the first position. When the receiver photosensor detected the rabbit in the receiver the program allowed a fixed delay time for the rabbit to settle down in the receiver. After the delay the block was moved to the second position where the extraction needle was inserted into the rabbit. After the chemistry was completed the needle was removed and the block moved to the third position where the rabbit dropped into a waste container. The block then returned to the first position.

This receiver was unsafe at any speed. After the receive step the rabbits frequently jammed either because the transport capsules were not at the bottom of the receiver or because the capsule cover had popped. At the third step the rabbit frequently failed to drop out of the receiver, again causing the receiver to jam. For long-lived nuclides these problems could be reduced to an acceptable leve:. For short-lived nuclides these problems were compounded by impact damage from moving the block too fast. This block weighed $2 \mathrm{~kg}$, which the pneumatic cylinder slammed very hard at all but the slowest speeds. This slamming jarred position sensors out of alignment resulting in progressive self-destruction. A less massive block was tried with no significant improvement. A further unavoidable problem was the excessive time required for the needle to penetrate the capsule. 


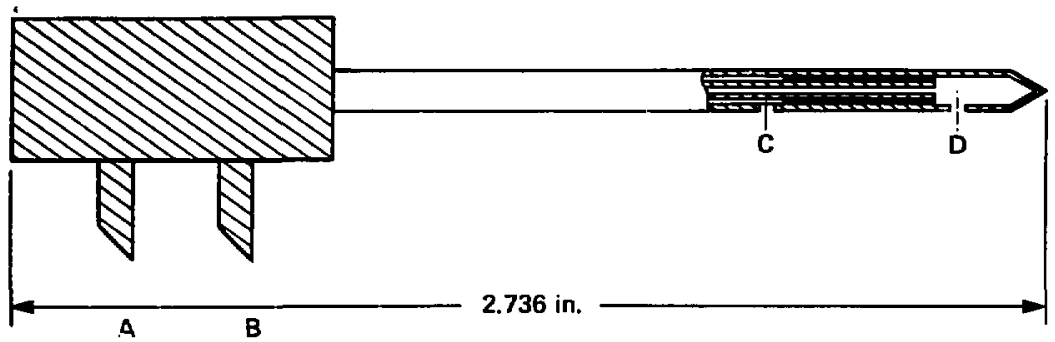

Fig. A1.1. The extraction needle. The outer part of the needle is a single piece, formed from hypodermic needle tubing. The tip is formed by cold flow in a dye. Tubes $A$ and $B$ are side arms for connecting to the rest of the system. Ports $C$ and $D$ are for extraction and venting respectively.

\section{New Receiver}

The new receiver shown in Fig. II.6 avoided the problems of the original receiver by having but one moving part, the flapper, and it moved only during the dump step. The new receiver was a box forming the end for the transport tube. The extraction needle was fixed upright in the bottom of the box. The rabbit's own momentum impaled it on the needle. The gas pressure behind the rabbit prevented its rebounding from the needle. When the sample was processed the happer was moved to the dump position and a blast of air ejected the rabbit to a waste container. This new receiver eliminated most of the faults of the old receiver, and when it did fail, it did not jam; it merely wasted a sample. The flapper was light and required motions could all be made when the moves were not time critical, greatly reducing the strain on the receiver.

\section{Needle}

The needle (shown in Fig. A1.1) was used to extract the liquid sample from the polyethylene sample capsule. The needle consists of a double concentric needle proper and a base with two side arms for connecting the needle to the chemistry apparatus. These pieces were silver-soldered together. The double concentric design was necessary to vent the sample capsule. Attempts were made using nitrogen pressure to help force the sample out. However, when the pressure was great enough to measurably speed the extraction, an unacceptable amount of the sample leaked around the needle and out of the capsule.

The only improvement to the original needle was to alter the construction of the exterior tube. Initially, for ease of construction, it was made in two pieces, a tip and a shank. The tip was formed by crimping the tube, giving a blade shape to the tip. Failures due to both these features were frequent. I converted to a single exterior piece with the tip closed in a conical dye. With these changes a netdle would last for 200 to $\mathbf{5 0 0}$ samples.

\section{Original Still}

The original still used in the hydride chemistry is shown in Fig. A1.2. It had two major drawbacks. Sparging was slow because of the size of the still $(-150 \mathrm{ml})$, because the liquid remained in a lump at the bottom of the still and because considerable fraction of the liquid remanned in the channels between the valves and the interior of the still. Slow sparging limited the speed of the chemist ${ }^{-y}$ and increased the noble gas contamination. 


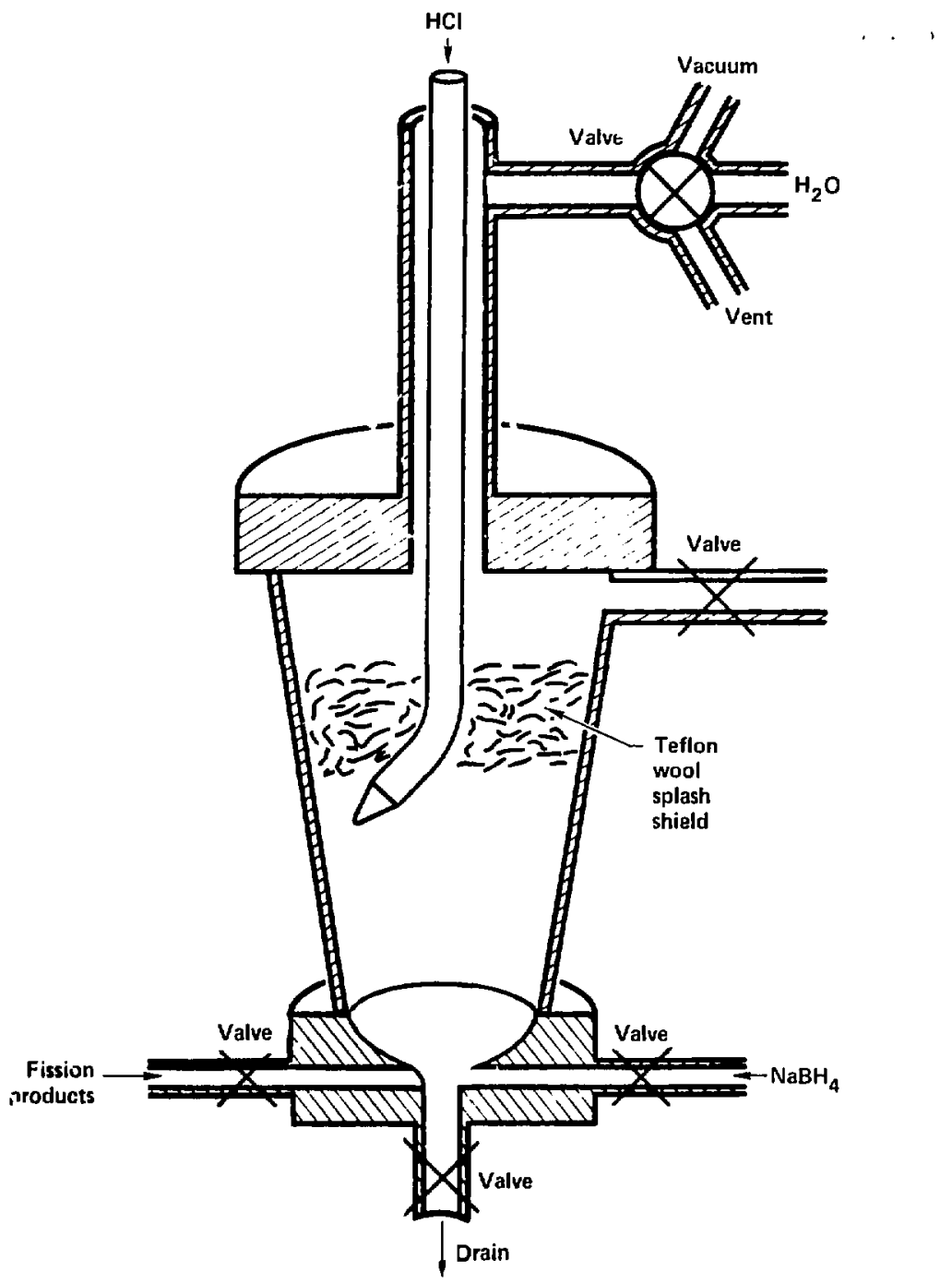

Fig. A1.2. One of the early versions of the hydride still. Total size and gravity separation of the gas-liquid mixture were problems to be overcome. 
Further, the gas-liquid separation was achieved by gravity alone. If the hyoride formation was not carefully controlled the gas-liquid mixture was blown out the hydride exit before separation. This ruined the current sample and soaked the stripping tube, requiring a replacement. These problems were both overcome with the new still and cyclone, described in Chapter II.

\section{Sample Cell}

The sample cell was made from a section of $6 \mathrm{~mm}$ polyethylene tubing. The sample cell was formed by heating the tubing and blowing a bubble of approximately $1 \mathrm{~cm}$ diameter. The bubble was then filled with a glass wool plug which was held in place by a piece of plastic "Chore-Boy". The sample cell was easy to make and when it falled it could easily be replaced. A more elaborate sample cell would have been more likely to leak, and being larger, would have presented greater difficulties to installation. 


\section{Appendix 2. Beta Decay of ${ }^{83} \mathrm{Se}^{\mathrm{m}}$}

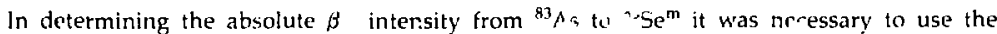
absolute gamma-ray intensities following the beta decay of ${ }^{\mathrm{k3}} \mathrm{Se}^{\mathrm{m}}$. Use of the tabulated values (NDS75a, TO178) resulted in an answer that was approximately 5 sigma negative for the $\beta$ intensity of interest. After careful cherking of my data and analysis revealed no errors, the source of the absolute gamma intensities were checked. The direct $(\mathrm{SCH} 68)$ and gamma deduced (MEY78, TOI78, NDS75a) beta intensities did not agree. Since the gamma-ray measurements give more accurate results the results were combined. Further investigation revealed the source of the error and resulted in an improved value for the absolute ground state intensity for the $\beta$ decay of "8? $\mathrm{Se}^{\mathrm{n}}$.

The direct $\beta$ intensity measurement (SCH68) yielded relative intensities of $31 \%, 34 \%$, and $35 \%$ to level groups at 0,1 , and $2 \mathrm{MeV}$. These relative intensities were compared to a decay scheme, showing good agreement. The relative intensities were then taken as absolute inte.nsities. Although the decays ' eme for ${ }^{\mathrm{k} \cdot} \mathrm{Se}^{\mathrm{m}}$ has changed radically since the $\beta$ measurement was made, the ground state is intensity is still cited as $31 \%$ absolute. Using this value, the 13 intensities deduced from a current decay scheme are $31 \%, 48 \%$, and $20 \%$ to the levels at 0,1 , and $2 \mathrm{MeV}$, where levels at $0.356,1.66$, and 2.88 have been ignored. The errors for these intensities are much less than the differences between the two sets of values.

The data sets can be brought into agreement by treating all of the intensities as relative and requiring that the observed $\beta$ intensity supply exactly the $\beta$ intensity deduced from the decay scheme (i.e. $34 \%=48 \%$ ) and disregard the direct $\beta$ intensity measurement to the levels at 2 MeY. This procedure yields an absolute ground state $\beta$ intensity of $(39 \pm 2) \%$ using the data from Ref. MEY78. The alsolute intensity to the level groups at 1 and $2 \mathrm{MeV}$ is then $43 \%$ and $18 \%$ respectively:

The error is due to failure to correct for the spectral contamination from ${ }^{k /}$ Se decay due to the ${ }^{R 0} \mathrm{Se}$ component in the enriched ${ }^{82}$ Se target. Schussler states that he irradiated an $89.1 \%$ enriched ${ }^{82}$ Se target in thermal neutrons for $2 \mathrm{~min}$., waited for $1.5 \mathrm{~min}$ fnr the $17 \mathrm{~s}$ activity due to ${ }^{77} \mathrm{Se}$ to decay away, then counted for two periods of 2 min. separated by 1 min., the second counting period to correct for long lived activity. He does not give an isotopic analysis of the target nor an explanation of how he corrected for the ${ }^{81}$ Se activity. Apparently, no correction was made for long lived contamination. From isotopic analyses of similar targets made at Oak Ridge National Laboratory, a reasonable analy'sis of Schusslers target would be $89.1 \%{ }^{82} \mathrm{Se}, 5.5 \%{ }^{\mathrm{R}} \mathrm{Se}, 3.4 \%{ }^{7 \mathrm{~S}} \mathrm{Se}, 0.9 \%$ ${ }^{77} \mathrm{Se}, 1.1 \%{ }^{76} \mathrm{Se}$, and $<.1 \%{ }^{74} \mathrm{Se}$ hased on the isotopic analysis of a sample $87.8 \%$ enriched ${ }^{12} \mathrm{Se}$. (Ref. ADA83). Only the ${ }^{\text {gl }} \mathrm{Se}$ contamination has a significant effect on the $\beta^{-}$spectrum. The expected relative intensitjes of the $1.585 \mathrm{MeV} B$ from ${ }^{81} \mathrm{Se}$ and the $1.87 \mathrm{MeV} B$ from ${ }^{83} \mathrm{Se}$ can be calculated from

$N^{\prime}=\frac{N_{0}(s \sigma \sigma}{\lambda}\left(1-e^{\lambda t_{1}}\right)\left(e^{\lambda t_{1}}-\mathrm{e}^{\lambda I_{1}}\right) I_{i d}$

Where $\Lambda_{0}^{\prime}$ is the number of target atoms, $\phi$ is the flux, $\sigma$ is the capture cross section, $\lambda$ is the decay constant, $t, i$, the irradiation time, $t$, and $t$, are the times of the beginning and ending of the counting period, and $I_{b}$ is the absolute $\beta$ intensity. Obtaining values for $\beta$ intensity for ${ }^{p /}$ Se, cross-section and half-life from Ref. TOI78, the intensity ratio of $1.58 \mathrm{MeV} \beta$ from ${ }^{\text {Bl }} \mathrm{Se}$ to the 1.87 MeV of from ${ }^{x / S e} e^{m}$ is 2.1. Comparing Schussler's work and the intensity deduced from the decay scheme, this ration is 1.5 .

This good agreement (considering the large uncertainties involved, i.e. $25 \%$ in the

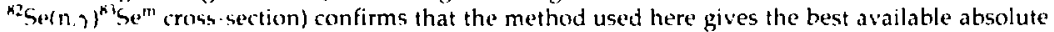
ground atate of intemate for the decay of ${ }^{33} \mathrm{Se}^{\mathrm{m}}$. 


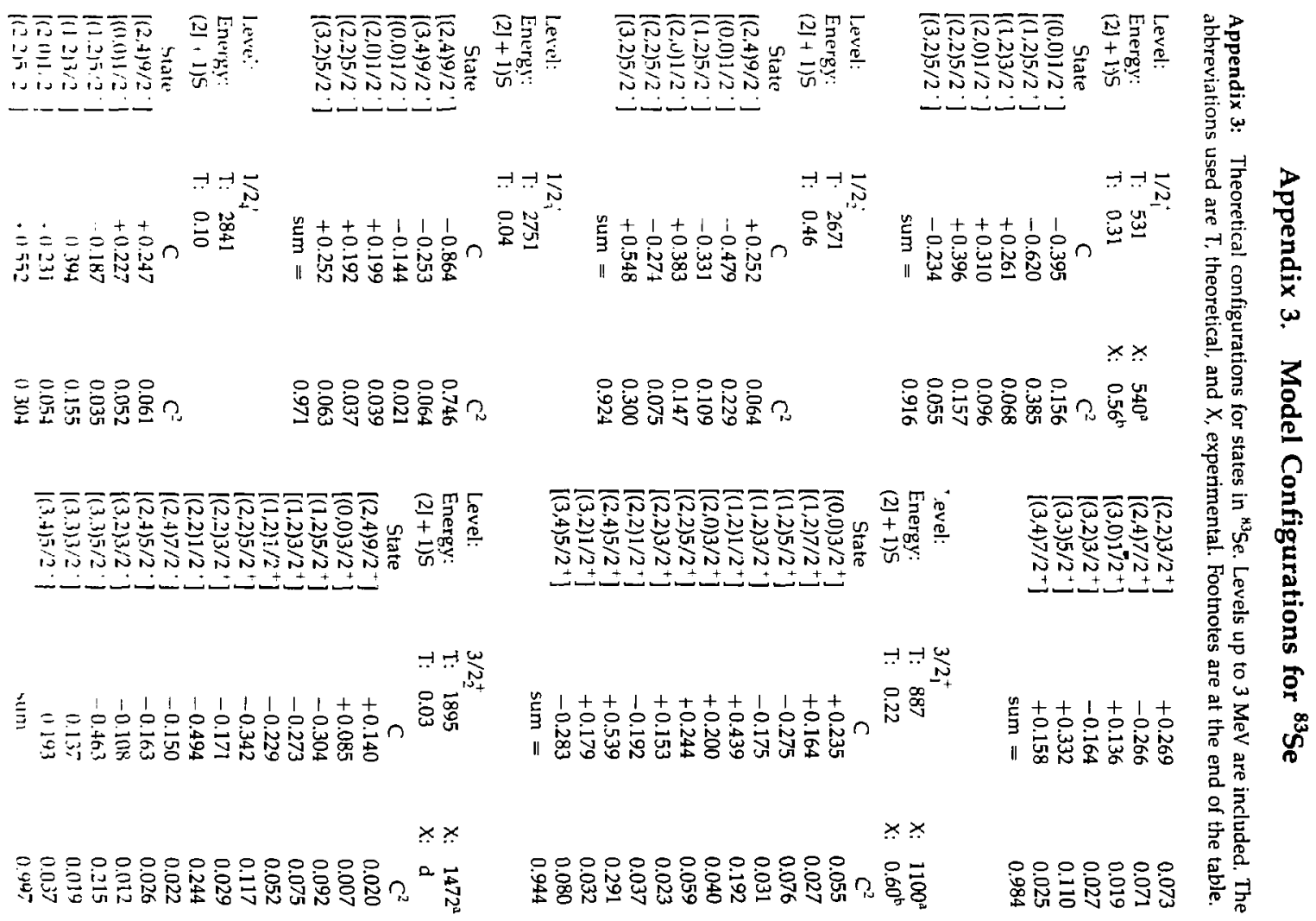




\begin{tabular}{|c|c|c|c|c|c|c|c|}
\hline Level: & $3 / 2{ }^{t}$ & & & {$\left[(1,2) 1 / 2^{+}\right]$} & -0.343 & & 0.117 \\
\hline Energy: & T: $\quad 2373$ & $\mathrm{X}:$ & $1710^{a}$ & {$\left[(2,0) 5 / 2^{+}\right]$} & -0.300 & & 0.090 \\
\hline$(2)+1) S$ & $\mathrm{~T}: \quad 0.03$ & & & {$\left[(2,2) 5 / 2{ }^{+}\right]$} & -0.121 & & 0.015 \\
\hline State & $C$ & & $C^{2}$ & {$[(2,2) 1 / 2+]$} & +0.210 & & 0.044 \\
\hline$\left[(2,4) 9 / 2^{+}\right]$ & +0.326 & & 0.106 & {$\left[(2,4) 5 / 2^{\prime}\right]$} & -0.277 & & 0.077 \\
\hline$\left[(0,0) 3 / 2^{+}\right]$ & -0.063 & & 0.004 & {$\left[(2,4) 3 / 2^{\circ}\right]$} & +0.177 & & 0.032 \\
\hline$[(1,2) 5 / 2]$ & -0.649 & & 0.422 & {$\left[(3,2) 5 / 2^{*}\right]$} & +0.158 & & 0.025 \\
\hline$[(1,2) 3 / 2+]$ & +0.190 & & $0 . \complement 36$ & {$[(3,2) 1 / 2+]$} & -0.133 & & 0.018 \\
\hline$[(1,2) 1 / 2+]$ & +0.128 & & 0.016 & {$\left[(3,4) 5 / 2^{+}\right]$} & +0.101 & & 0.010 \\
\hline$[(2,2) 5 / 2 \cdot]$ & +0.421 & & 0.177 & & $\operatorname{sum}=$ & & 0.949 \\
\hline$[(2,2) 3 / 2 \star j]$ & -0.189 & & 0.056 & & & & \\
\hline$[(2,4) 7 / 2\}$ & +0.181 & & 0.033 & Level: & $5 / 2 i$ & & \\
\hline$\left[(2,4) 5 / 2^{+}\right]$ & -0.248 & & 0.061 & Energy: & T: 1378 & $X:$ & $1331^{\circ}$ \\
\hline$\{(3,3) 5 / 2+\}$ & +0.175 & & 0.031 & $(2 J+1) S$ & T: $\quad 0.00$ & $x:$ & $0.33^{\mathrm{b}}$ \\
\hline$[(3,4) 7 / 2$ & -0.125 & & 0.016 & State & C & & $C^{2}$ \\
\hline$[(3,4) 5 / 2 \cdot]$ & +0.172 & & 0.030 & {$\left[(1,2) 9 / 2^{\prime}\right]$} & +0.909 & & 0.827 \\
\hline & sum $=$ & & 0.967 & {$\left[(2,2) 9 / 2{ }^{\prime}\right]$} & +0.234 & & 0.055 \\
\hline & & & & {$[(2,4) 9 / 2 ']$} & +0.114 & & C.013 \\
\hline Level: & $3 / 2 i^{\circ}$ & & & {$[(1,2) 5 / 2]$} & -0.208 & & 0.043 \\
\hline Energy: & T: 2861 & & & {$[(2,2) 5 / 2]$} & -0.149 & & 0.022 \\
\hline$(2 \mathrm{~J}+1) \mathrm{S}$ & $\mathrm{T}: \quad 0.02$ & & & {$\left[(2,2) 1 / 2^{\prime}\right]$} & -0.125 & & 0.016 \\
\hline State & C & & $c^{2}$ & & sum $=$ & & 0.975 \\
\hline$\left[(2,4) 9 / 2^{\circ}\right]$ & +0.887 & & 0.787 & & & & \\
\hline$[(3,4) 9 / 2\}$ & +0.225 & & 0.050 & Level: & $5 / 2{ }^{i}$ & & \\
\hline$[(0,0) 3 / 2 !$ & -0.071 & & 0.005 & Energy: & $\mathrm{T}: \quad 1551$ & $X:$ & $1665^{\prime \prime}$ \\
\hline$[(1,2) 5 / 2]$ & +0.200 & & 0.040 & $(2 \mathrm{~J}+1) \mathrm{S}$ & $\mathrm{T}: \quad 0.09$ & $x:$ & $0.38^{\mathrm{b}}$ \\
\hline$\{(2,2) 5 / 2\}]$ & -0.201 & & 0.041 & State & C & & $c^{2}$ \\
\hline$[(2,2) 1 / 2]$ & +0.115 & & 0.013 & {$\left[(1,2) 9 / 2^{\circ}\right]$} & -0.242 & & 0.059 \\
\hline$[(2,4) 5 / 2]$ & +0.159 & & 0.025 & {$\left[(0,0) 5 / 2^{+}\right]$} & +0.124 & & 0.015 \\
\hline & sum $=$ & & 0.961 & {$\left[(1,2) 5 / 2^{+}\right]$} & -0.346 & & 0.120 \\
\hline & & & & {$\left[(1,2) 1 / 2^{+}\right]$} & -0.360 & & 0.130 \\
\hline Level: & $3 / 2 \xi^{\circ}$ & & & {$\left[(2,2) 5 / 2^{+}\right]$} & -0.589 & & 0.347 \\
\hline Energy: & T: 2985 & $x:$ & $2535^{a}$ & {$\left[(2,2) 1 / 2^{+}\right]$} & -0.218 & & 0.048 \\
\hline$(2 J+1) S$ & $\mathrm{~T}: \quad 0.78$ & $x:$ & $1.98^{\mathrm{h}}$ & {$\left[(2,4) 5 / 2^{+}\right]$} & 0.187 & & 0.035 \\
\hline State & $C$ & & $c^{2}$ & {$\left[(2,4) 3 / 2^{+}\right]$} & +0.196 & & 0.039 \\
\hline$\left[(0,0) 3 / 2^{+}\right]$ & -0.442 & & 0.196 & {$\left[(3,0) 5 / 2^{+}\right]$} & +0.118 & & 0.014 \\
\hline$\left[(1,2) 7 / 2^{+}\right]$ & -0.405 & & 0.164 & {$[(3,2) 1 / 2+]$} & -0.147 & & 0.022 \\
\hline$[(1,2) 3 / 2 \cdot j$ & +0.238 & & 0.057 & {$\left[(3,3) 5 / 2^{+}\right]$} & -0.329 & & 0.108 \\
\hline$[(2,0) 3 / 2]$, & -0.273 & & 0.074 & {$\left[(3,3) 3 / 2^{+}\right]$} & +0.122 & & 0.015 \\
\hline$\{(2,2) 7 / 2+\}$ & +0.207 & & 0.043 & {$\left[(3,4) 5 / 2^{+}\right]$} & +0.109 & & 0.012 \\
\hline$[(2,2) 5 / 2+]$ & +0.189 & & 0.036 & & sum $=$ & & 0.962 \\
\hline$\left[(2,2) 1 / 2^{+}\right]$ & -0.258 & & 0.067 & & & & \\
\hline$[(2,4) 7 / 2+]$ & +0.155 & & 0.024 & Level: & $5 / 2 t^{\dagger}$ & & \\
\hline$\left[(2,4) 5 / 2^{+}\right]$ & +0.233 & & 0.054 & Energy: & T: 2184 & $X:$ & $2482^{\prime \prime}$ \\
\hline$\left[(3,2) 7 / 2^{+}\right]$ & -0.144 & & 0.021 & $(2 J+1) S$ & $\mathrm{~T}: \quad 2.77$ & $\mathrm{x}:$ & $0.47^{k}$ \\
\hline$[(3,4) 5 / 2+]$ & -0.475 & & 0.226 & State & C & & $C^{2}$ \\
\hline & sum $=$ & & 0.960 & {$\left[(1,2) 9 / 2^{\dagger}\right]$} & -0.115 & & 0.013 \\
\hline & & & & {$[(0,0) 5 / 2+]$} & -0.700 & & 0.491 \\
\hline Level: & $5 / 2 i$ & & & {$[(2,0) 5 / 2\}$} & +0.431 & & 0.186 \\
\hline Energy: & T: $\quad 588$ & $x:$ & $582^{\prime \prime}$ & $[(2,2)] / 2\rfloor$ & .0 .203 & & 0.041 \\
\hline$(2)+1) 5$ & $\mathrm{~T}: \quad 1.59$ & $\mathrm{X}$ : & $2.76^{b}$ & $|(2,4) 5 / 2|$ & +0.235 & & 0.055 \\
\hline State & $c$ & & $c^{2}$ & {$[(3,2), 7 / 2]$} & 0.351 & & 0123 \\
\hline$[(1,2) 9 / 2]$ & .0159 & & 0.025 & {$[(3,2) 12]$} & $\cdot 11165$ & & 01127 \\
\hline$[(0,0) 5 / 2]$ & 11315 & & 0.265 & {$[(3+1)=2\}$} & 11115 & & 4013 \\
\hline (1) $215=1$ & $.0+61$ & & 9231 & & sum -- & & $114+4$ \\
\hline & & & & & & & \\
\hline
\end{tabular}




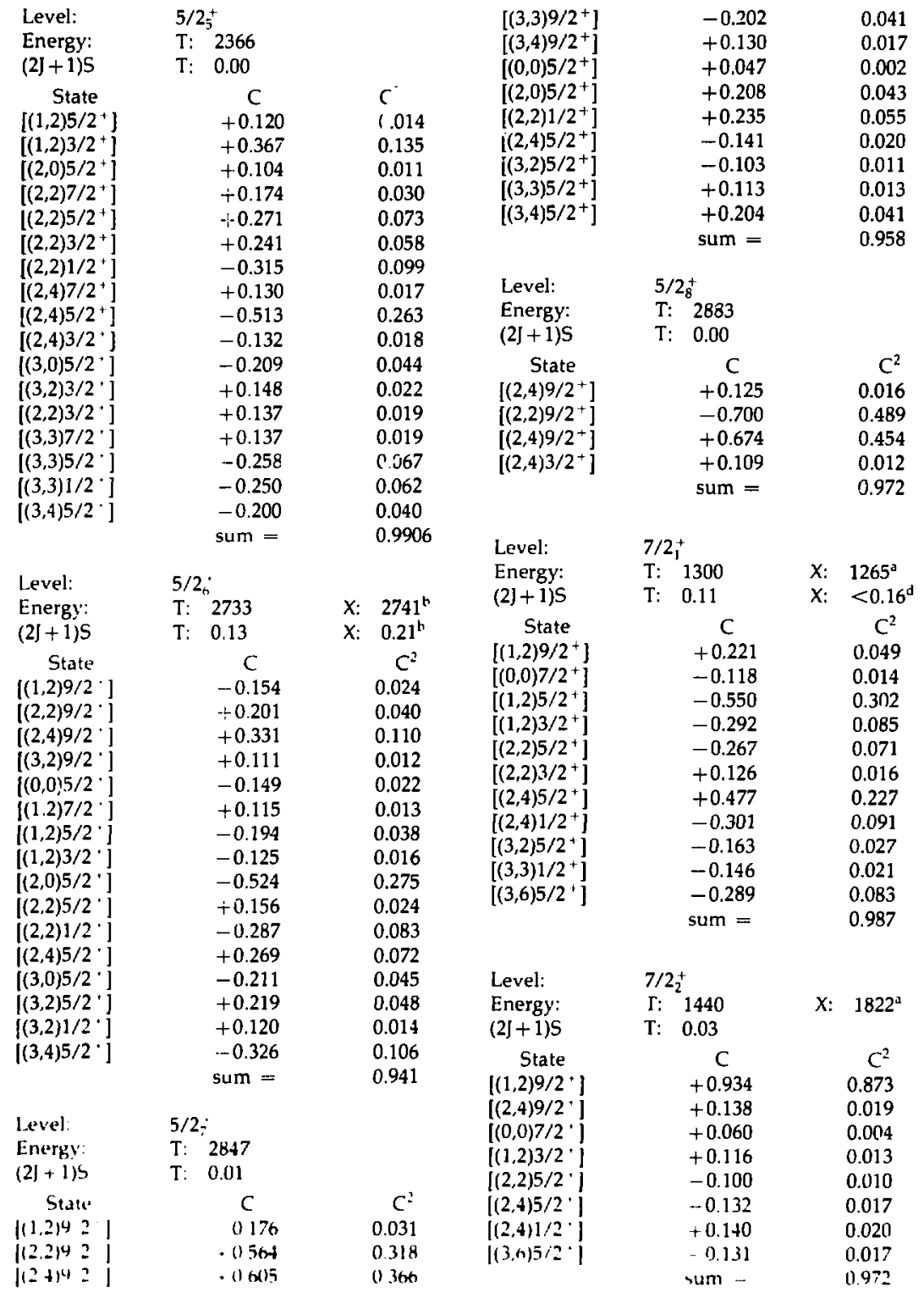




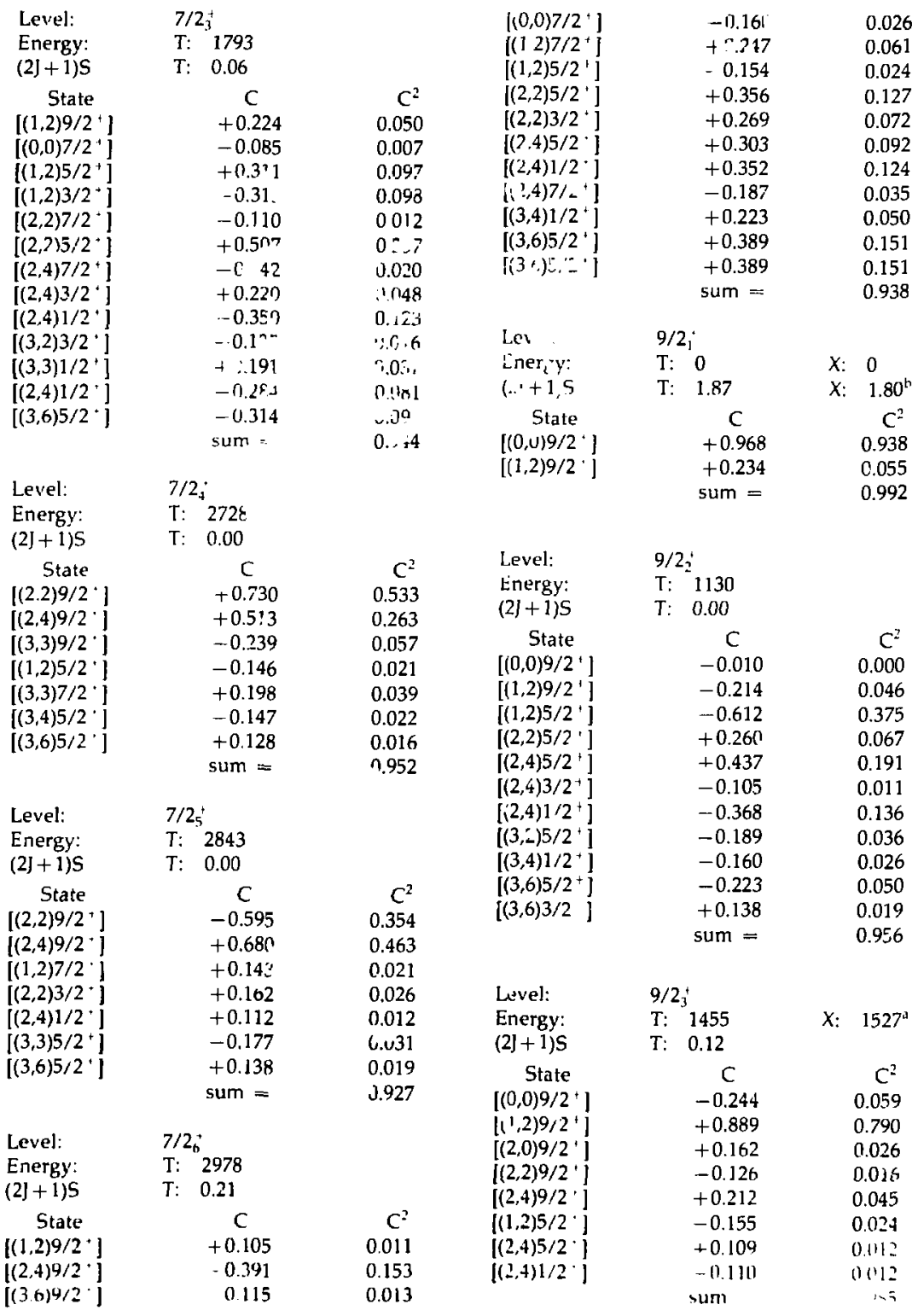




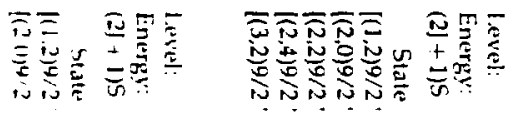

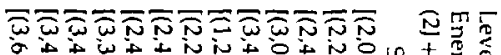

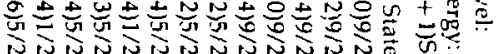

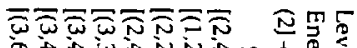

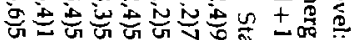

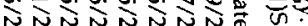

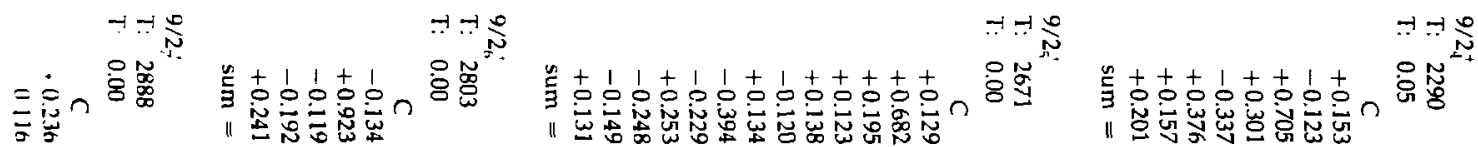

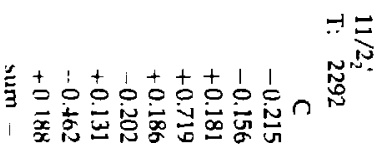

0000000000

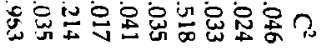

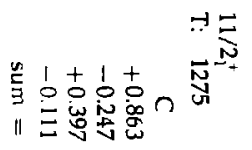

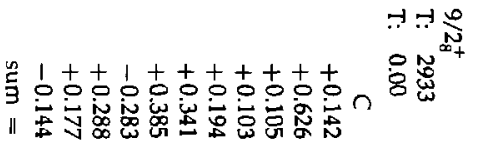

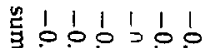

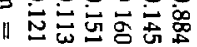

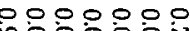

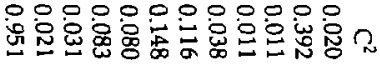

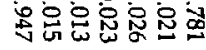




\begin{tabular}{|c|c|c|}
\hline $\begin{array}{l}\text { Level: } \\
\text { Energy: }\end{array}$ & $\begin{array}{l}1 / 2\}^{\prime} \\
{[: \quad 2850}\end{array}$ & \\
\hline $\begin{array}{c}\text { State } \\
{\left[(2,2) 9 / 2^{+}\right]}\end{array}$ & $\begin{array}{c}C \\
+0.716\end{array}$ & $\begin{array}{r}C^{2} \\
0.513\end{array}$ \\
\hline$[(2,4) 9 / 2$ & +0.421 & 0.177 \\
\hline$[(3,4) 9 / 2+]$ & +0.164 & 0.027 \\
\hline$[(3,6) 9 / 2+]$ & +0.168 & 0.028 \\
\hline$\left[(1,2) 7 / 2^{+}\right]$ & +0.186 & 0.035 \\
\hline$[(2,4) 3 / 2+]$ & +0.250 & 0.062 \\
\hline$[(3,3) 5 / 2+]$ & +0.172 & 0.030 \\
\hline$[(3,4) 5 / 2+]$ & -0.232 & 0.054 \\
\hline$[(3,6) 5 / 2 \cdot]$ & -0.151 & 0.023 \\
\hline \multirow[t]{2}{*}[(3,6)1/2^{\circ}]{} & +0.125 & 0.016 \\
\hline & sum = & 0.965 \\
\hline
\end{tabular}

Level: $\quad 11 / 2$;

Energy:

State

[(1,2)9/2 ']

$[(2,2) 9 / 2$ ']

$[(2,4) 9,: \cdot]$

$[(3,2) 9 / 2 *]$

$[(3,4) 9 / 2 \cdot]$

$\left[(3,6) 9 / 2^{*}\right]$

$[(3,3) 5 / 2 *]$

Level:

Energy:

State

$[(2,2) 9 / 2$.

$[(2,4) 9 / 2+j$

$[(3,2) 9 / 2$ '

$[(3,3) 7 / 2+]$

Level:

Energy:

State

$\left[(2,2) 9 / 2^{+}\right]$

$[(2,4) 9 / 2$ ']

$[(3,2) 9 / 2+]$

$[(3,4) 9 / 2 \cdot]$

$[(3,3) 7 / 2 \times]$

$[(3,6) 7 / 2+]$

$[(3,6) 3 / 2+\}$

$[(3,6) 1 / 2$,

$[(1,2) 9 / 2]$

1.?201

Intergy

State

[(2.)4) |
T: 3006

$\begin{array}{ccc}C & C^{2} & {[(3,3) 7 / 2} \\ -0.477 & 0.227 & {[(3,6) 7 / 2+]} \\ -0.271 & 0.073 & {[(3,6) 3 / 2} \\ +0.649 & 0.421 & {[(1,2) 9 / 2]}\end{array}$

13/2;

[: 1306

$\begin{array}{cc}C & C^{2} \\ +0.867 & 0.751 \\ +0.207 & 0.043 \\ +0.332 & 0.110 \\ -0.259 & 0.067 \\ \text { sum }= & 0.972\end{array}$

$13 / 2$

T: 2219

$$
\text { C }
$$

$+0.101$

$+0.190$

$+0.311$

$+0.140$

$+0.723$

$-0.231$

$-0.374$

$+0.107$

$+0.305$

sum $=$

$13 / 2$ i

厂 2otor

(

(1) 78
Level:

Energy:

$(2]+1) S$

State

$\left[(3,2) 9 / 2^{\prime}\right]$

$\left[(3,4) 9 / 2{ }^{\prime}\right]$

$\left[(3,6) 9 / 2^{\dagger}\right]$

$\left[(2,4) 7 / 2^{+}\right]$

$[(3,6) 7 / 2 \cdot]$

Level.

Energy:

State

$\left[(2,2) 9 / 2^{\prime}\right]$

$\left[(2,4) 9 / 2^{\prime}\right]$

$[(3,2) 9 / 2$ ']

[(3,3) $9 / 2$ ']

$[(3,4) 9 / 2$ ']

$[(2,4) 7 / 2$ ']

$[(3,3) 7 / 2$ ']

$[(3,6) 3 / 2]$

0.042

0.066

0.100

0.947

$[(0,0) 1 / 2]$

$[(1,2) 3 / 2]$

$[(1,2) 5 / 2]$

Level:

Energy:

State

$[(0,0) 1 / 2$

$[(1,2) 3 / 2$

$[(1,2) 5 / 2$

$[(2,0) 1 / 2]$

$[(2,2) 3 / 2]$

$[(2,2) 5 / 2]$

$[(3,2) 3 / 2$ ]

$[(3,2) 5 / 2]$

0.523

0.054 ।

0.140

0.011

0.093

0.984

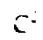

(1) $\mathrm{inl}$

Level:

Energy:

State

[(1,2)3/2

[(1.2)5/2

$[(2,0)\}, 2$

|122)5?
$-0.481$

$+0.226$

$+0.317$

$-0.205$

$-0.111$

sum $=$

$13 / 2$;

T: 3030

$$
\text { C }
$$

$-0.472$

$+0.376$

$+0.575$

$+0.209$

$+0.199$

$+0.325$

$-0.253$

$-0.120$

$+0.130$

$-0.138$

sum $=$

0.231

0.051

0.100

0.042

0.012

0.997

$c^{2}$

0.222

0.141

0.330

0044

0.039

0.105

0.064

0.014

0.017

0.019

0.997

$1 / 2$.

T: $\quad 229$

T. 229

X: 229

$\mathrm{X}: \quad 0.11$

C

$+0.956$

$C^{2}$

$+0.190$

$-0.215$

0.914

0.036

0.046

sum $=$

0.996

$1 / 2_{2}$

T: 2387<smiles>C</smiles>

$+0.249$

$\mathrm{C}^{2}$

$-0.671$

0.062

0.450

$+0.345$

0.119

$-0.516$

0.266

$-0.216$

0.047

$+0.162$

0.026

$-0.108$

0,012

$+0.119$

0.014

sum $=$

0.937
$1 / 2$;

T: 2639

$\begin{array}{cc}C & C^{2} \\ +0.609 & 0.371 \\ +0727 & 0.529 \\ 11235 & 0.155 \\ +1116 . & 51126 \\ .4 m & 0145:\end{array}$




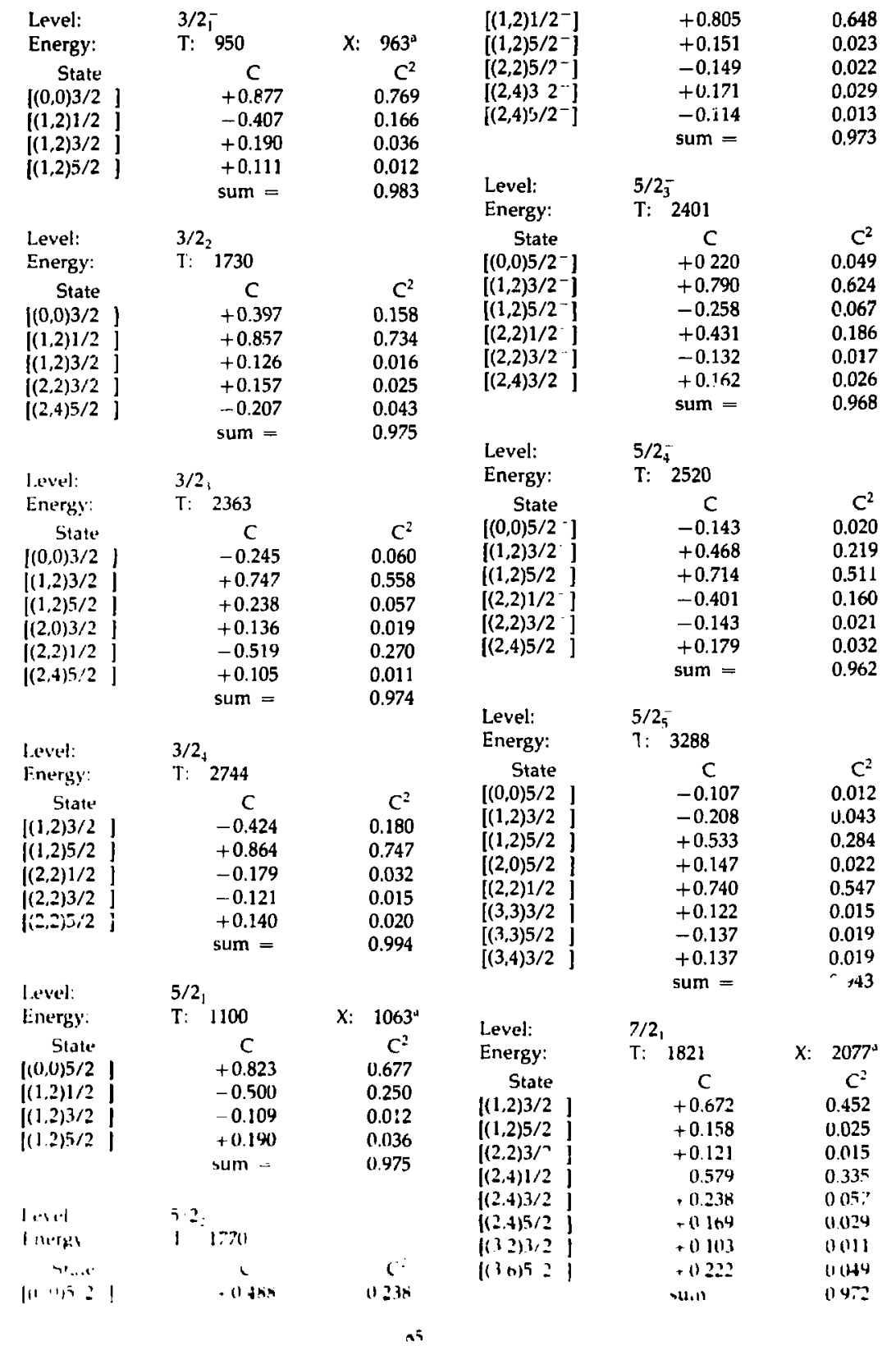




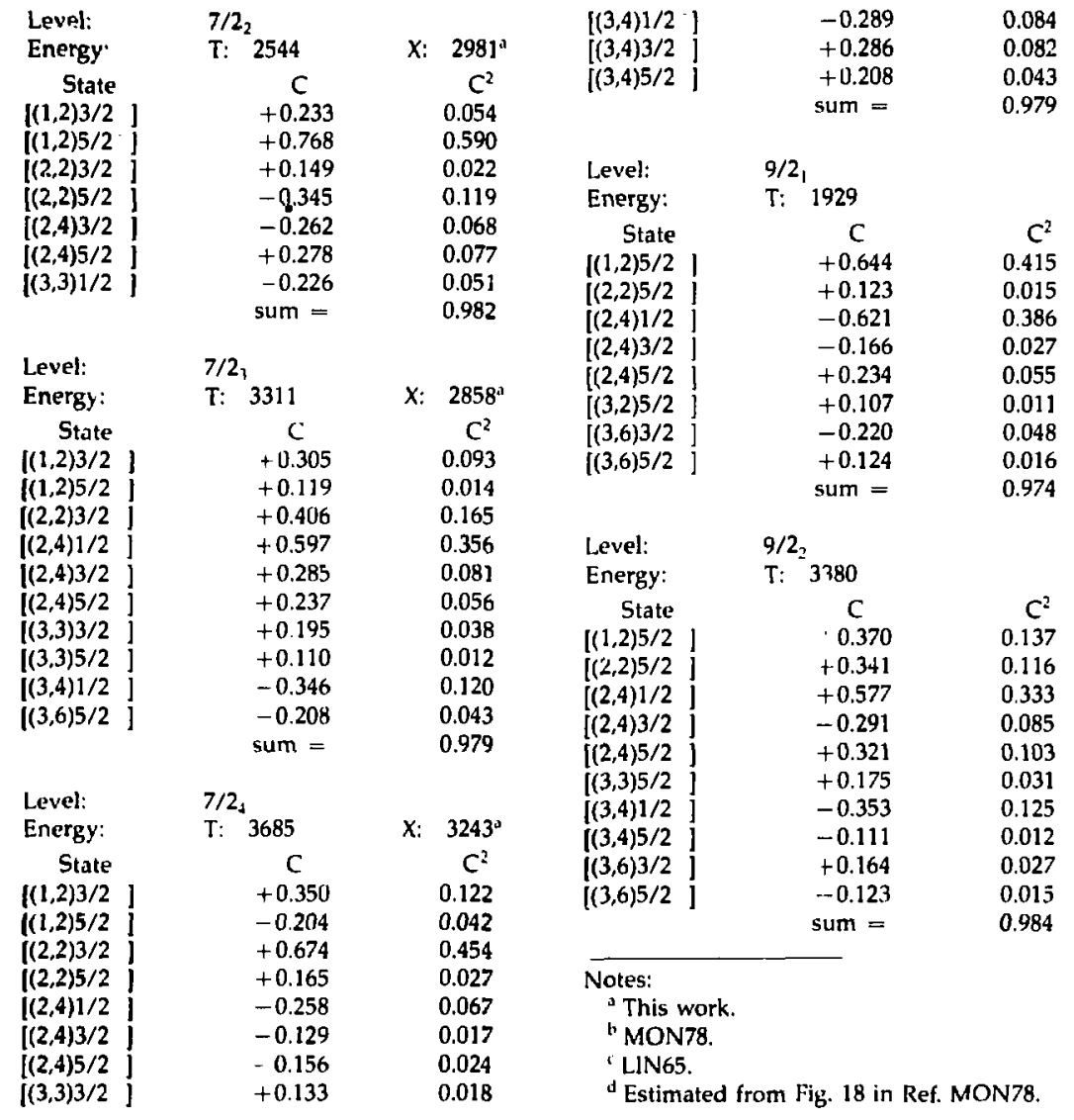




\section{References}

ADA83 Adair, $H$., private communication The isotopic analysis of an $87.8 \%$ enriched ${ }^{82}$ Se sample produced at Oak Ridge Nationa! Laboratory in 1964.

BAL71 Ball, J. B., Nucl. Phys. A160, 225 (1971).

"Studie; of ${ }^{95} \mathrm{Ru}$ and ${ }^{94} \mathrm{Ru}$ with the ${ }^{96} \mathrm{Ru}(\mathrm{p}, \mathrm{d})$ and $(\mathrm{p}, \mathrm{t})$ Reactions."

BOH75 Bohr, A. and B. Mottleson, Nuclear Structurc (Benjamin, New York, 1975), vol. 2, ch. 5.

CHO54 Choudhury, D. C., Dan. Mat. Fys. Medd. 28 (1954).

"Inter'nediate Coupling Calculations in the Unified Model."

FET82 Fettweis, 1., P. del Marmol, M. Degreef, P. Duhamel, and J. Vanhorenbeeck, Z. Phys. A305, 57 (1982).

"Excited States in ${ }^{97} \mathrm{Pd} . "$

FOL69 Folger, H., J.-V. Kratz and G. Herrmann, Radiochem. Radioanal. Lett. L, 185 (1969).

"Rapid Volatilization of Arsenic, Selenium, Antimony, and Tellurium in Form of Their Hydrides."

FRI65 Friedlander, G. J. W. Kennedy, and J. M. Miller, Nuclear and Radiochemistry, 2nd Ed., John Wiley and Sons, Inc., New York, 1965. p. 76.

GLA79 Glascock, M. D., E. W. Schneider, W. B. Walters, S. V. Jackson, and R. A. Meyer, Phys. Rev. C20, 2370 (1979).

"Level structure of $0{ }^{\prime \prime}$ mass $1 \quad$ i and the unified model. II. ${ }^{117}$ In levels populated in the decay of ${ }^{117} \mathrm{Cd}$ isol

GRO68 Groshev, I.. V., A. M. Demidov, V. I. Pelekhov, L. L. Sokolooskii, G. A. Bartholomew, A. Deveika, K. M. Eastwood, and S. Monaro, Atomic Data and M M,clear Data Tables 5. 1 (1968).

"Compendium of Thermal-Neutron-Capture $\gamma$-Ray Meas'srements."

GUN72 Cunnink, R., and J. B. Niday, UCRL-51061, 1972. Esprecially Vol. 1., pp. 4-..5, “Data Reduction."

"Computerized Quantitative Analysis by Gamma-ray Spectroscopy."

GUS81 Gustafsson, H.-A. T. Bjornstad, B. Jonson, O.C. Jonsson, V. Lindfors, S. Mattsson, A. M. Poskanzer, H. L. Ravn and D. Schardt, Procecdings of the 4 th Conference on Nicle' Lar irom Stability, Helsingor, 704 (1981).

"Comparative Yields of Alkali Metals and Thallium from Uranium Irradiated with High-energy Protons, ${ }^{3} \mathrm{Ho}$ and ${ }^{12} \mathrm{C} . "$

HAR72 Harar, S., and R. N. Horoshko, Nucl. Phys. A183, 161 (1972).

"Study of the Level Scheine of ${ }^{117}$ In via Proton Transfer Reactions."

HER69 Herrmann, G. and H. O. Denschlag, Ann. Rev. Nucl. Sci., 19, 12 (1969).

"Rapid Chemical Separation."

HEY78 Heyde, K., M. Waroquier, and R. A. Meyer, Phys. Rev. C17, 1219 (1978).

Unified description of odd-mass indium nuclei: I. General theory and comparison to

${ }^{113}$ In and ${ }^{115} \mathrm{In}$ levels populated in the decay of ${ }^{113} \mathrm{Sn}$ and ${ }^{115} \mathrm{Sn}^{\mathrm{m} . \mathrm{y}}$."

H.) BO Heyde K. M Waroquier and $P$. Van Isacker, Phys. Rev. C22, 1267 (1980).

Lintfied de's ription of odd-mass indium nuclei: III. Application to 11.121 In."

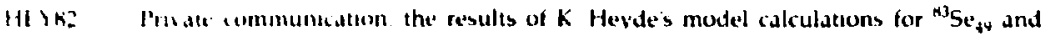

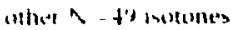


HOF81 Hoff, P., K. Aleklett, B. Folgelberg, E. Lund and G. Rudstam, Proceedings of the 4 th Conference on Nucloi Far from Stability, Helsingor, 704 (1981).

"Decay properties of ${ }^{B 1} \mathrm{Ga}$ and ${ }^{\mathrm{Q}} \mathrm{Ge}$ and Observation of Abnormal Energy Shift in the P1/2 State."

HOF82 Hoff, Г., privute commurication.

KON66 Konopinski, E. J., The The'ory of Beta Rndionctivity, Oxford University Press, 1966, p. 157

KRA70 Kratz, J - V. and G. Herrmann, J. Ino"g. Nucl. Chem. 32, 3713 (1970).

"Halfives, Fission Yields and Neutron Emission Probabilities of ${ }^{87} \mathrm{Se},{ }^{88} \mathrm{Se}$ and Evidence for ${ }^{87} \mathrm{As}$."

KRA75 Kratz, J. V., H. Franz, N. Kaffrell, and G. Herrmann, Nucl. Phys. A250, 13 (1975).

"Gamma-ray Emission from ${ }^{80.86}$ As Isotopes."

LAN79 Lane, S. M., Thesis, Univ. of Calif. at Davis (1979).

"The Nuclear Structure of Tellurium 133 via Beta Decay and Shell Model Calculations in the Doubly Magic Tin 132 Region."

LED71 Lederer, C. M., J. M. laklevic, and I. M. Hollander, Nucl. ithys. A169, 489 (1971).

"In-beam Gamm 1-Ray Spectroscopy of Odd Neutron Mo and Ru Isotopes."

LIE79 Lien, III, O. C.. High speed motion picture of the LLNL. still and cyclone separator.

LIE80 Lien, III, O. G. Г. C. Stephenson, E. A. Henry, R P. Yaffe, and R. A. Meyer, Nucl. Instrum. Meth. 185, 351 (1981).

"AUTOBATCH: I. System Description for the Automated Batchwisr Jsolation and Study of Short-Lived Fission Products."

LiN65 Lin, E. K., Phys. Rev. 139, 8340 (1965).

"Nuclear St, victure Studies in the Selenium Isotopes with $(d, p)$ and $(d, t)$ Reartions."

1.IN80 Lin. J., E. A. Henry and R. A. Meyer, UCRL-84536, submitted to the Sixth Conference on Application of Accelerators in Research and Industry, Denton, Texas, 1980.

"Detection Efficiency of Ge(Li) and HPGe D tors for Gamma rays up to 10-MeV."

MAR74 Markham, R. G. and H. W. Fulbright, Phys. Rev. C9, 1633,1974).

"Structure of ${ }^{113}$ In Studied by the Reactions ${ }^{115} \ln (p, t)^{113} \mathrm{In},{ }^{112} \mathrm{Cd}\left({ }^{3} \mathrm{He}, \mathrm{d}\right)^{113} \mathrm{In}$, and

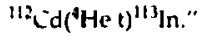

MASBO Private communication. T. N. Massey.

MAT77 Matsuki S., N. Sakamoto, K. Ogino, Y. Kadota, Y. Saito, T. Tanabe, M. Yasue, and Y. Okuma. Phys. Lett. 72B, 310 (1978).

"Systematics of Low Lying Octupole States in the Doubly-Even Nuclei from Ge to Sr."

MEY78 Meyer, R.A., LLNL publication M-100 (1978).

"Multigamma-ray Calibration Standards."

MEY79a Meyer, R. A., Lawrence Livermore National Laboratory, Livemore, California, Preprint UCRL-81069 (1979); presented at the Workshop on Nuclear Spectroscopy of Fission Products, Grenoble, France, May 1979.

"Rapid Automartu Nuclear Chemistry."

MEY7ub Meyer, R A., E. A. Henry, O. C. Lien, III, H. C. Griffin, S. M. Lane, R. P. Yaffe, P C.

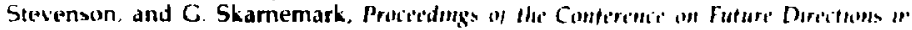

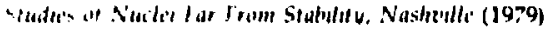

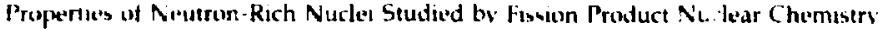


MEY81 Private communication from R. A. Meyer, the results of gamma spectroscopy following the beta decay of ${ }^{83} \mathrm{Se}^{\mathrm{m}}$ from ${ }^{82} \mathrm{Se}(n, \gamma)$.

MON78 Montestruque, L. A., M. C. Cobian-Rozak, G. Szaloky, J. D. Zumbro, and S. E. Darden, Nucl. Phys. A305, 29 (1978).

'Study of the ${ }^{77,79,81.83}$ Se Level Structure with the ${ }^{76,78,80,82} \mathrm{Se}(\overrightarrow{\mathrm{d}}, \mathrm{p})$ Reaction."

NDS75 Nuclea Data Sheets, 16, 445 (1975). $A=89$.

NDS79 Nuclear Data Sheets, 27, 389 (1979). $A=87$.

NDS80a . Nuclear Data Sheets, 30, 413 (1980). A $=115$.

NDS80b Nuclear Data Sheets, 34, $v$ (1981).

REE,O Reehal, B. S., and R. A. Sorenann, Phys. Rev. C2, 819 (1970).

"Electric Quadrupole Transitions in Odd-Mass Spherical Nuclei."

ROS78 Rosel. F., H. M. Fries, K. Alder, and H. C. Pauli, Atomic and Nuclear Data Tables, 21, 385 (1985).

"Internal Conversion Coefficients for all Atomic Shells."

SCH68 Schussler, F., Le Journal de Physique, 29, 385 (1968).

"Measurement of the beta transition intensities in the ${ }^{83} \mathrm{Se}^{m}$ by magnetic spectrometer."

STE7; Stevenson, P. C., private communication, program written for a Data General, Inc. NOVA 1200 min compuler.

STO80 $\quad ?_{1} \cdots$ te communication, description of computer code FITEK.

Srm81 Symons, T. J. M., Procetedings of the 4 th Conference an Nuclei Far from Stability, Helsilgur, b68 (1981).

"Production of Nuclei Far from Stability by Fragmentation of High Energy Heavy lons."

THU70 Thuriere, E., Thesis, IJniversity of Paris, as cited in Ref. NDS80a.

TIT77 Tittel, G., N. Kaffrell, N. Trautmann, and G. Herrmann, J. Inorg. Nucl. Chem. 39. 2115 (1977).

"Direct Identification of ${ }^{103-107}$ Mo by a Rapid Chemical Separation Procedure"

TRA7B N. Trautmann on a review of fast chemistry, in Proceedings of the American Chemical Sorctety. Murm (1978).

TOl78 Talli' of Jatophe's, 7th Ed., C. M. Lederer and V. S. Shirley, Eds., John Wiley and Sons., 1978.

VIN82 Vincront, C. H. NIMS 203, 307 (1982).

"The Estimation of Differential Counting Measurements of Positive Quantities with Relatively large Statistical Errors."

IVLY Weiffenbach, C., S. C. Gujrathi, and J. K. P. Lee, Can. J. Phys. 53, 101 (1975).

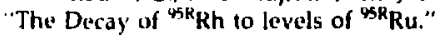

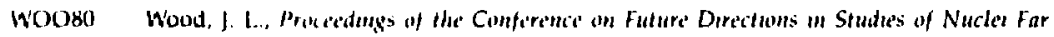
frum stollulat v. Nishillli. 37 (1979).

The $\mathrm{U}_{\mathrm{st}^{2}}$ of Si'stematics in the Interpretation of Nuclear Structure Far from the BetaStable Regilen 

Gasphase fur Spaltproduct."

ZGA80 Zgangar, E. F., Proceddings of the Conference on Future Directions in Studies of Nucloi Far from Stability. Nashoille, 49 (1979).

"The Structure of Neutron Deficient Odd-Proton Nuclei Near the Shell Closure at $Z=82$.

\section{Acknowledgments}

I would like to thank all of the penple who contributed to my thesis. In a project of this scope one person could not do it alone. The following people made special contributions to this thesis. Discussions with the late P. C. Stevenson on nuclear chemistry were instrumental in the final form of AUTOBATCH. R. A. Meyer had the concept that nuclear chemistry could compete with isotope separation, and I also thank him for his continued support throughout my thesis project. Discussions with E. A. Henry greatly enlarged my understanding of gamma-ray spectroscopy. R. $\Gamma$. Yaffe for introducing me to radio chemistry and showing me the discipline necessary for completing this thesis. For facilities and financial support, 1 am grateful to $C$. Gatrousis of the Nuclear Chemistry Division of Lawrence Livermore Natonal Laboratory and to G. L. Struble head of the Nuclear Properties Group. I would like to thank S. D. Bloom for introducing me to theoretical muclear phy'sics and K. Heyde for making the model calculations for ${ }^{83} \mathrm{Se} .1$ greatly appreciate the long hours worked by the reactor personnel which made this experiment possible. Finally, I must thank H. S. Chesnutt for his indispensable help in turning the AUTOBATCH concept into reality. 